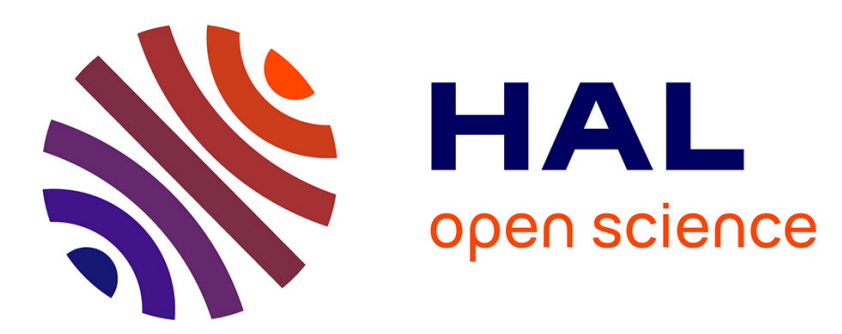

\title{
Measurement of CIB power spectra over large sky areas from Planck HFI maps
}

\author{
Daisy Suet Ying Mak, Anthony Challinor, George Efstathiou, Guilaine \\ Lagache
}

\section{- To cite this version:}

Daisy Suet Ying Mak, Anthony Challinor, George Efstathiou, Guilaine Lagache. Measurement of CIB power spectra over large sky areas from Planck HFI maps. Monthly Notices of the Royal Astronomical Society, 2017, 466 (1), pp.286-319. 10.1093/mnras/stw3112 . hal-01792585

\section{HAL Id: hal-01792585 \\ https://hal.science/hal-01792585}

Submitted on 9 Jul 2019

HAL is a multi-disciplinary open access archive for the deposit and dissemination of scientific research documents, whether they are published or not. The documents may come from teaching and research institutions in France or abroad, or from public or private research centers.
L'archive ouverte pluridisciplinaire HAL, est destinée au dépôt et à la diffusion de documents scientifiques de niveau recherche, publiés ou non, émanant des établissements d'enseignement et de recherche français ou étrangers, des laboratoires publics ou privés. 


\title{
Measurement of CIB power spectra over large sky areas from Planck HFI maps
}

\author{
Daisy Suet Ying Mak, ${ }^{1 \star}$ Anthony Challinor, ${ }^{1,2 \star}$ George Efstathiou ${ }^{1}$ \\ and Guilaine Lagache ${ }^{3}$ \\ ${ }^{1}$ Institute of Astronomy and Kavli Institute for Cosmology Cambridge, Madingley Road, Cambridge CB3 OHA, UK \\ ${ }^{2}$ DAMTP, Centre for Mathematical Sciences, Wilberforce Road, Cambridge CB3 OWA, UK \\ ${ }^{3}$ Aix Marseille Université, CNRS, LAM (Laboratoire d'Astrophysique de Marseille) UMR 7326, F-13388 Marseille, France
}

Accepted 2016 November 28. Received 2016 November 17; in original form 2016 September 20

\begin{abstract}
We present new measurements of the power spectra of the cosmic infrared background (CIB) anisotropies using the Planck 2015 full-mission High frequency instrument data at 353, 545 and $857 \mathrm{GHz}$ over $20000 \mathrm{deg}^{2}$. We use techniques similar to those applied for the cosmological analysis of Planck, subtracting dust emission at the power spectrum level. Our analysis gives stable solutions for the CIB power spectra with increasing sky coverage up to about 50 per cent of the sky. These spectra agree well with $\mathrm{H}$ I-cleaned spectra from Planck measured on much smaller areas of sky with low Galactic dust emission. At 545 and $857 \mathrm{GHz}$, our CIB spectra agree well with those measured from Herschel data. We find that the CIB spectra at $\ell \gtrsim 500$ are well fitted by a power-law model for the clustered CIB, with a shallow index $\gamma^{\text {cib }}=0.53 \pm$ 0.02. This is consistent with the CIB results at $217 \mathrm{GHz}$ from the cosmological parameter analysis of Planck. We show that a linear combination of the 545 and $857 \mathrm{GHz}$ Planck maps is dominated by the CIB fluctuations at multipoles $\ell \gtrsim 300$.
\end{abstract}

Key words: galaxies: star formation-cosmology: observations-large-scale structure of Universe - infrared: diffuse background.

\section{INTRODUCTION}

The cosmic infrared background (CIB) arises as the integrated emission from the dust heated by starlight in star-forming galaxies. The CIB carries a wealth of information about the growth of galaxies and hence the process of star formation. The most informative method to study the CIB is to resolve the individual sources and then perform analyses of clustering and counts on the resolved sources. However, the high density of faint, distant galaxies makes this very challenging, particularly at the lower frequencies seen by Planck which are expected to probe the highest redshift sources. In fact, only 10 per cent of the CIB has been resolved into galaxies by Herschel at $857 \mathrm{GHz}$ (Béthermin et al. 2010; Oliver et al. 2010) and negligibly so for Planck with its poorer angular resolution (Planck Collaboration VII 2013). An alternative approach, and the only one feasible at lower frequencies in the foreseeable future, is to study the statistical properties of the unresolved background. Correlated anisotropies in the background reflect the clustering properties of the unresolved galaxies and so are a powerful probe of large-scale structure (e.g. Haiman \& Knox 2000). On large scales, the angular power spectrum of galaxy clustering should reflect the underlying distribution of dark matter haloes (the

\footnotetext{
^E-mail: dmak@ast.cam.ac.uk (DSYM); adc1000@ast.cam.ac.uk (AC)
}

two-halo term) and hence halo bias; on smaller non-linear scales pairs of galaxies within the same parent halo become an important contribution to the signal (the one-halo term). The CIB also depends on the mean emissivity per comoving volume and so probes models of galaxy evolution, star formation and the initial stellar mass function.

CIB fluctuations have been measured at $3300 \mathrm{GHz}$ (AKARI; Matsuura et al. 2011) and $3000 \mathrm{GHz}$ [Infrared Imaging Surveyor (IRAS)/IRIS; Pénin et al. 2012; Planck Collaboration XXX 2014e], $1875 \mathrm{GHz}$ (Spitzer; Grossan \& Smoot 2007; Lagache et al. 2007), $600 \mathrm{GHz}$ with Ballon-borne Large Aperture Submillimeter Telescope (Viero et al. 2009; Hajian et al. 2012) and Herschel/Spectral and Photometric Imaging Receive (SPIRE) (Amblard et al. 2010; Viero et al. 2013), $220 \mathrm{GHz}$ with Atacama Cosmology Telescope (Dunkley et al. 2011) and South Pole Telescope (Hall et al. 2010) and at 217, 353, 545 and $857 \mathrm{GHz}$ by Planck (Planck Collaboration XVIII 2011a, hereafter PEP, and Planck Collaboration XXX 2014e, hereafter P13). The results in PEP are based on power spectrum measurements over a total of $140 \mathrm{deg}^{2}$. P13 extends this to $2200 \mathrm{deg}^{2}$, in the cleanest regions of the sky for which $\mathrm{H}_{\mathrm{I}}$ data, used as a tracer to subtract Galactic dust emission from the CIB, is available. The mean redshift of the CIB is expected to increase with decreasing frequency, with models suggesting that over 90 per cent of the clustering power at scales of $5 \mathrm{arcmin}$ in the 353 and $217 \mathrm{GHz}$ Planck bands comes from $z>2$ (PEP). Planck data are therefore 
able to place strong constraints on models of large-scale structure, galaxy evolution and star formation at high redshift via CIB measurements.

Accurate removal of Galactic dust emission is critical for measurements of CIB clustering. At present, almost all measurements of CIB power spectra either assume that the power spectrum of Galactic dust emission can be described by a simple power-law template (e.g. $C_{\ell} \propto \ell^{-2.8}$ ) or use relatively low-resolution (around $10^{\prime}$ full width at half-maximum, FWHM) $\mathrm{H}_{\mathrm{I}}$ maps as a tracer of Galactic dust emission in regions of low $\mathrm{H}$ I column density to subtract dust in the map domain at low multipoles, together with a power-law template in the power spectrum domain (fitted to the $\mathrm{H}$ I power spectrum on larger angular scales) to subtract dust emission at higher multipoles. However, comparison of Planck maps with $\mathrm{H}$ I observations reveals excess dust emission at $\mathrm{H}$ i column densities greater than $2.5 \times 10^{20} \mathrm{~cm}^{2}$, probably caused by the formation of molecular hydrogen in dusty regions (see Planck Collaboration XXIV 2011b). H i cleaning is therefore possible only in the cleanest regions of the sky.

Here, we take a different approach to separate Galactic dust emission from the CIB over large areas of the sky. We do this by exploiting the statistical isotropy of the extragalactic signals to construct dust power spectrum templates from differences of spectra measured over different areas of the sky. This approach is very similar to that adopted for the CMB likelihood analysis by the Planck team (Planck Collaboration XV 2014c; Planck Collaboration XI 2016b). The amplitudes of the Galactic dust templates at the three frequencies that we consider in this paper $(353,545$ and $857 \mathrm{GHz})$ are fitted as part of the likelihood analysis of the six frequency (cross)spectra. Similarly, we model the cosmic microwave background (CMB) anisotropies at the power spectrum level rather than using component separation techniques to produce a cleaned CMB map. Our approach to cleaning the CIB spectra through model fitting therefore differs from previous analyses of the Planck data in PEP and P13, which used map-based removal of Galactic dust and the CMB.

Our model fitting approach can be applied to large areas of the sky and can therefore be used to test the stability of the recovered CIB power spectra as a function of sky coverage. This is the primary aim of this paper. The downside is that the dust and CMB lead to a high sampling variance compared to subtraction of these components at the map level. Removing an estimate of our realization of the CMB at the map level on large scales would improve the CIB constraints at $353 \mathrm{GHz}$ (and at lower frequencies). This is the approach taken in P13, where $100 \mathrm{GHz}$ Planck maps were used as a CMB template. However, this requires modelling of additional components at $100 \mathrm{GHz}$, particularly thermal Sunyaev-Zel'dovich (SZ) emission and also modelling the mismatch of the beam profiles at $100 \mathrm{GHz}$ and higher frequencies. Removing Galactic dust emission at the map level requires a tracer, e.g. H I as in P13. However, as discussed above, using $\mathrm{H}$ I data limits the sky coverage and angular resolution. An alternative is to use internal estimates of dust emission (see Planck Collaboration XLVIII 2016e for recent progress with the Planck data). The difficulty with this approach lies in separating CIB and dust with the limited frequency coverage of the Planck data and quantifying accurately any residuals.

This paper is organized as follows. Section 2 describes the Planck data and sky masks used in this paper. The parametric models that we adopt for the CIB and our modelling of foreground components are discussed in Section 3. Details of the power spectra measurements and construction of the likelihood are given in Section 4. In that section, we also present tests to validate the likelihood framework and discuss the expected degeneracies between parameters. ${ }^{1}$ In Section 5, we show the main results from our likelihood analysis, presenting parameter constraints for the CIB and dust foregrounds and comparing our results with those of previous measurements. Section 6 develops a modification to the dust contribution to the covariance matrix of the measured power spectra and explores whether Galactic dust emission can be removed by forming a linear combination of the 545 and $857 \mathrm{GHz}$ Planck maps. Our conclusions are summarized in Section 7. The appendices give further details about the structure of the covariance matrix of the measured power spectra and our likelihood validation (Appendix A), the data sets and modelling used to make predictions for the Poisson power from source counts (Appendix B) and our attempts to account for the statistically anisotropic nature of Galactic dust in the covariance matrix of the power spectra (Appendix C). Appendix D presents additional tests of the stability of our results.

Throughout the paper, we adopt the standard Lambda cold dark matter $(\Lambda \mathrm{CDM})$ cosmological model as our fiducial cosmology, with parameter values derived from the $\mathrm{CMB}$ power spectra as measured by Planck. Specifically, we adopt the Planck TT+lowP values from table 4 of Planck Collaboration XI (2016b).

\section{DATA SETS AND SKY MASKS}

\subsection{Planck maps}

The results in this paper are based on the Planck 2015 full-mission frequency maps at the three highest frequencies: 353, 545 and $857 \mathrm{GHz}$. More specifically, we use yearly maps for this analysis; these are constructed by combining Surveys 1 and 2 to form the Year-1 map and Surveys 3 and 4 to form the Year-2 map (see table A.2 in Planck Collaboration VIII 2016a). Power spectra are estimated by cross-correlating Year-1 and Year- 2 maps. As the instrument noise is very nearly uncorrelated between the yearly maps, we make no further correction for noise bias in the estimated power spectra. We consider alternative splits of the data in Appendix D, including half-mission maps and those made from detector sets.

The maps are calibrated on the CMB orbital dipole for $353 \mathrm{GHz}$ and on planets for 545 and $857 \mathrm{GHz}$. For $353 \mathrm{GHz}$, the calibration is accurate to about 0.78 per cent. At 545 and $857 \mathrm{GHz}$, the errors on the absolute calibration is about 6 per cent and the relative calibration between the two channels is better than 3 per cent. We include map-level calibration parameters in our likelihood analysis with Gaussian priors reflecting the errors on the absolute calibration. ${ }^{2}$ Conservatively, we do not impose the more precise relative calibration of the $353-857 \mathrm{GHz}$ channels in our priors. Compared to the maps in the 2013 Planck release, the calibration factors changed by 1.9 per cent and 4.1 per cent at 545 and $857 \mathrm{GHz}$, respectively, which is within the planet modelling uncertainty. The combination of these, and other changes to the data processing (such as beam

\footnotetext{
${ }^{1}$ Throughout this paper, we use the cosmomc package (Lewis \& Bridle 2002; http://cosmologist.info/cosmomc) to perform Markov chain Monte Carlo (MCMC) exploration of the likelihood.

${ }^{2}$ Note, however, that recent work on the relative cailbration between the $545 \mathrm{GHz}$ channel and the CMB channels of Planck, based on the Solar dipole and the first acoustic peak, show that this relative calibration is, in fact, correct to within 1.5 per cent (see Planck Collaboration XLVI 2016d). The accuracy of the absolute calibration of the $545 \mathrm{GHz}$ maps is therefore also accurate to this level, given the precise absolute calibration of the $\mathrm{CMB}$ channels off the orbital dipole.
} 
characterization and corrections for non-linearities in the on-board analogue-to-digital conversion), lead to the 2015 frequency maps being less bright by 1.8 per cent at $545 \mathrm{GHz}$ and 3.3 per cent at $857 \mathrm{GHz}$ compared to those in the 2013 release (Planck Collaboration VIII 2014a). The $353 \mathrm{GHz}$ maps in the 2015 release are brighter by 2.1 per cent compared to the 2013 release. Maps are given in units either of megaJansky per steradian (with the photometric convention $v I_{v}=$ const.) or $K_{\mathrm{CMB}}$. The conversion between the two can be computed exactly given knowledge of the bandpass filters. Throughout this paper, we convert measurements in $K_{\mathrm{CMB}}$ units to megaJansky per steradian units using the mean coefficients: $287.45(353 \mathrm{GHz}), 58.04(545 \mathrm{GHz})$ and $2.27(857 \mathrm{GHz})$ as given in $\mathrm{P} 13$.

We estimate the noise properties of the Planck maps from the differences of maps constructed from the first and second half of each ring period (i.e. the half-ring half-difference maps; Planck Collaboration VIII 2016a). These maps provide a good statistical representation of the noise in the actual sky maps. We compute the noise spectra from these maps, which we use in combination with the hit-counts to construct the noise model used in the likelihood analysis.

The maps are provided in HEALPIX ${ }^{3}$ format, with resolution parameter $N_{\text {side }}=2048$, corresponding to pixels with a typical width of $1.7 \mathrm{arcmin}$. Unless otherwise specified, all the maps are plotted using a Mollweide projection centred on the Galactic Centre with the Galactic plane horizontal. In analysing these maps, we use the fiducial beam transfer functions described in Planck Collaboration VIII (2016a). Our primary goal is to compute the six (cross-)frequency spectra by cross-correlating the yearly maps at 353545 , and $857 \mathrm{GHz}$. In forming cross-spectra at different frequencies, e.g. $353 \times 545$, we average the Year- $1 \times$ Year- 2 spectra, discarding the Year- $1 \times$ Year- 1 and Year- $2 \times$ Year- 2 spectra. We measure cross-spectra using the CAMSPEC software, described in the context of CMB power spectrum estimation in Planck Collaboration XV (2014c). Briefly, pseudo-spectra are computed from masked sky maps and are subsequently deconvolved for the mode-coupling effect of the mask and beam and pixel effects. The covariance matrices for these spectra are calculated with analytic approximations that account for masking and the statistically anisotropic nature of the Planck instrument noise. Our interpretation of the spectra is based on fitting simple parametrized models of the CIB and foreground components in an MCMC likelihood analysis, as presented in Section 3. The signal power in the best-fitting models is used to construct the sample variance in the covariance matrices of the power spectra. We also interpret our results in the context of halo models using the CIB template spectra adopted in P13. This allows us to compare and test the quality of our fits of the Planck 2015 data directly against the analysis of the 2013 data carried out by the Planck team in P13.

\subsection{Sky masks for the galactic region and bright sources}

We mask regions of strong Galactic emission and bright point sources before estimating power spectra. A key part of our analysis is to demonstrate that our results are stable as a function of sky coverage. We do this by considering a set of Galactic masks as described below.

\footnotetext{
${ }^{3} \mathrm{http}: / /$ healpix.sourceforge.net
}

\subsubsection{Galactic masks}

We use a set of Galactic masks that are obtained by thresholding a smoothed CMB-cleaned $353 \mathrm{GHz}$ map at different levels in order to obtain different desired sky fractions. All of the Galactic masks are apodized with a $2^{\circ}$ FWHM Gaussian to reduce modecoupling caused by sharp mask boundaries. In this paper, most of our results are based on unmasked sky fractions of 30 percent, 40 per cent and 50 per cent, corresponding to effective sky fractions after apodization of 24.5 per cent, 33.8 per cent and 42.8 per cent. ${ }^{4}$ For our baseline results, we use the 40 per cent mask. As we show in Section 3.3, there are small changes in the shape of the inferred dust power spectrum as we extend the sky coverage beyond 60 per cent associated with the anisotropy of the point source masks (which increases dramatically as the Galactic plane is approached) and small changes in the properties of Galactic dust. These, combined with the large increase in the amplitude of the dust power spectrum, limit the accuracy with which we can model and remove the dust power over larger sky fractions. It is important to note that as the sky fraction is increased, the sample variations in the dust power spectrum also increase. The optimal sky fraction for the CIB analysis presented here therefore involves a trade-off between improved signal-to-noise at small scales, where Galactic dust is sub-dominant compared to the $\mathrm{CIB}$, versus increased sample variance at large scales where Galactic dust dominates. ${ }^{5}$ Unless otherwise specified, we name the masks by the percentage of the sky that they retain before apodisation, e.g. mask 40 corresponds to the 40 per cent unmasked sky fraction.

\subsubsection{Point source masks}

We remove bright point sources using a mask constructed from compact sources identified in the Planck Catalogue of Compact Source (PCCS; Planck Collaboration XXVIII 2014d). In the Planck analysis of cosmological parameters, point sources detected in the PCCS with a signal-to-noise $\mathrm{S} / \mathrm{N}>7$ were masked and the remaining Poisson point source levels at $100-217 \mathrm{GHz}$ were treated as nuisance parameters in the likelihood analysis. For the analysis presented here using the $353-857 \mathrm{GHz}$ channels, we have constructed point source masks with a higher detection threshold and completeness level.

We use the PCCS as an entry catalogue, but select only those sources that satisfy a number of criteria to ensure that the majority are extragalactic (e.g. having counterparts in external catalogues, not being in regions of bright cirrus or molecular clouds). We validate the criteria using the observations of large Galactic fields by Herschel/SPIRE. The flux cut is about 400, 600 and $1000 \mathrm{mJy}$ at 353,545 and $857 \mathrm{GHz}$, respectively. We call these the 'frequencydependent masks'. We also construct a 'union mask' by combining the three frequency-dependent masks and use this as our baseline point source mask. We explore the effects on the CIB constraints when using these two versions of the point source masks in Appendix D.

The point source masks are apodized with a Gaussian of FWHM 30 arcmin and combined with the apodized Galactic masks to form the final masks used in the power spectrum analysis. Examples are shown in the upper panels of Fig. 1. Here the left- and right-hand

\footnotetext{
${ }^{4}$ We use a 25 per cent Galactic mask for calculating dust template spectra.

${ }^{5}$ This behaviour could be corrected by more careful weighting of the data during power spectrum estimation. Here, we weight the data uniformly after application of the mask.
} 

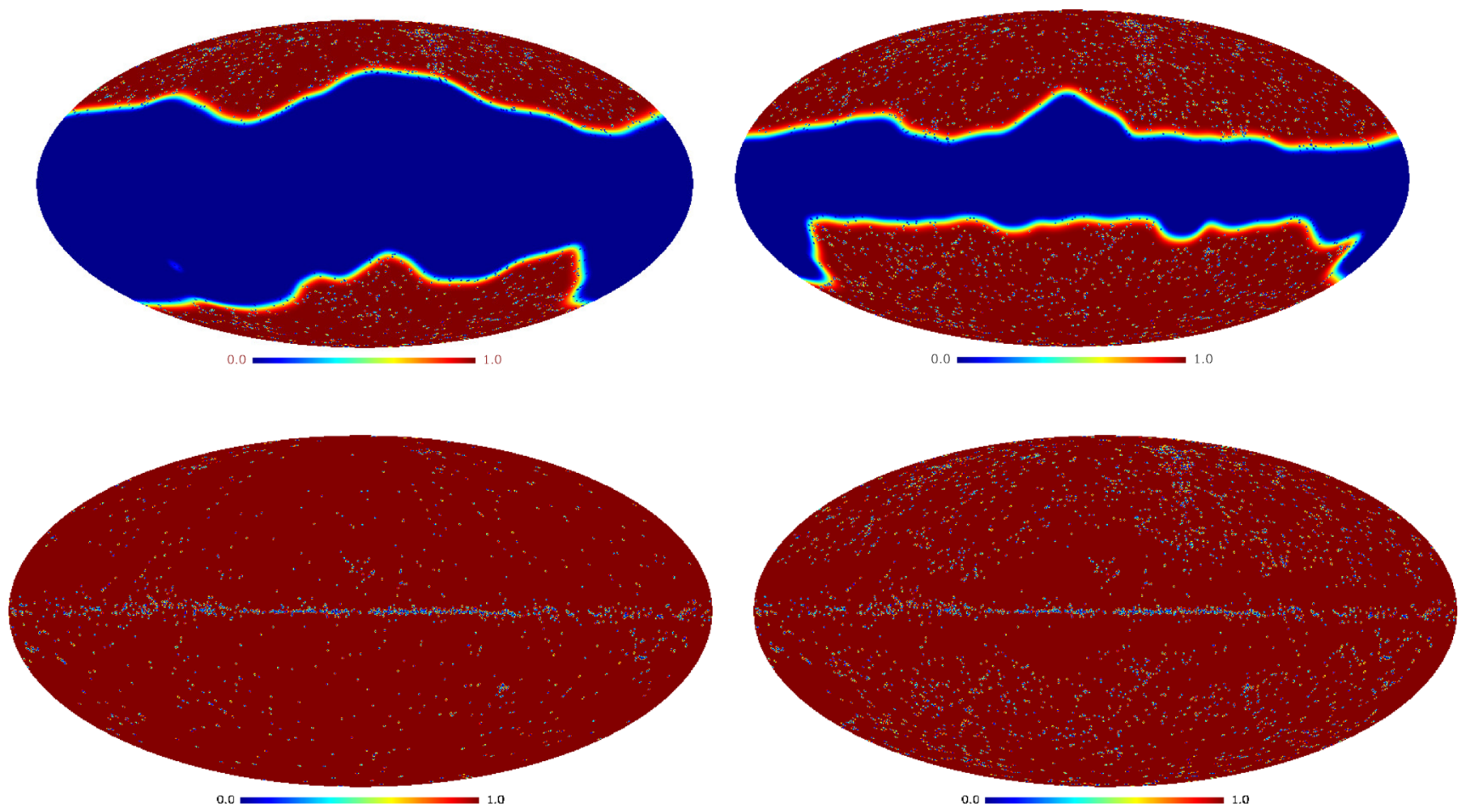

Figure 1. Upper: apodized Galactic mask 40 (left) and mask 70 (right) combined with the union point source mask adopted in this paper. Lower: $353 \mathrm{GHz}$ point source mask (left) and $857 \mathrm{GHz}$ point source mask (right).

panels show Galactic mask 40 and 70 , respectively, together with the union point source mask. Because we apply a dust background criterion, the point source masks become incomplete for sky fractions greater than around 60 per cent. This is illustrated by the lower panels in Fig. 1 that show the 353 (left) and $857 \mathrm{GHz}$ (right) point source masks. Note that the union mask is dominated by sources detected at $857 \mathrm{GHz}$.

\section{PARAMETRIC MODEL OF THE POWER SPECTRA}

We model the theoretical angular power spectrum for frequencies $v$ and $v^{\prime}$ as

$\mathcal{D}_{\ell}^{\mathrm{th}, v \times v^{\prime}}=\mathcal{D}_{\ell}^{\mathrm{clu}, \mathrm{cib}, \nu \times v^{\prime}}+\mathcal{D}_{\ell}^{\mathrm{dust}, v \times v^{\prime}}+\mathcal{D}_{\ell}^{\mathrm{cmb}, v \times v^{\prime}}+\mathcal{D}_{\ell}^{\mathrm{ps}, v \times v^{\prime}}$,

where e.g. $\mathcal{D}_{\ell}^{\text {th }, v \times v^{\prime}} \equiv \ell(\ell+1) C_{\ell}^{\text {th, } v \times v^{\prime}} / 2 \pi$. The terms in equation (1), in order, are the clustered contribution from the CIB, Galactic dust, primordial CMB and the Poisson power from infrared sources, respectively. The CMB signal is significant only at $353 \mathrm{GHz}$, though we include it at all frequencies in our analysis. The above decomposition separates the CIB into clustered and Poisson components. The sum of these components is well constrained by the Planck data, but the decomposition into clustered and Poisson components is partially degenerate with extent that depends on the multipole range used and the assumed shape of the clustered CIB power spectrum. Most of the statistical power of the Planck spectra comes from multipoles $\ell \lesssim 1500$, where we find clear evidence that the power spectrum of the clustered CIB component is shallower than the Poisson component. We therefore solve for the Poisson amplitudes in our likelihood analysis, which can then be compared with the expected Poisson levels computed from source counts (see Section 3.4).
In the following sections, we describe our parametrizations of the individual contributions in equation (1). The model parameters and prior ranges are summarized in Table 1.

\subsection{Clustered CIB}

\subsubsection{Power-law CIB model}

Following Planck Collaboration XV (2014c), we consider a powerlaw model for the CIB signal

$\mathcal{D}_{\ell}^{\text {clu,cib }, v \times v^{\prime}}=A_{v \times v^{\prime}}^{\text {cib }}\left(\frac{\ell}{2000}\right)^{\gamma^{\text {cib }}}$,

where $A_{v \times v^{\prime}}^{\text {cib }}$ is the amplitude of the clustered CIB power at multipole $\ell=2000$ in the $v \times v^{\prime}$ spectrum and $\gamma^{\text {cib }}$ is the power-law index. Our analysis of the Planck data is mostly sensitive to the clustered CIB around $\ell \approx 1000$ and, as we shall see, a simple power-law model provides a reasonably good description of the Planck spectra.

Theoretical work based on the halo model (see Section 3.1.2) suggests that the power-law index $\gamma^{\text {cib }}$ should steepen on smaller scales $(\ell \gtrsim 2000)$ where non-linear clustering becomes significant. Since the inferred value of $\gamma^{\text {cib }}$ is expected to depend on scale, care is needed in comparing our results for the power-law model with others in the literature. For high- $\ell$ CMB experiments (e.g. Story et al. 2013; Dunkley et al. 2013), which have sensitivity to the nonlinear clustering regime, the power-law index is usually held fixed at $\gamma^{\text {cib }}=0.8$, as inferred from measured galaxy correlation functions at optical wavelengths (e.g. Zehavi et al. 2002).

PEP reported values of $\gamma^{\text {cib }} \approx 0.82-0.96$ from fits to the Planck autopower spectra in the multipole range $\ell=200-2000$. However, in that analysis, the Poisson power levels were fixed to the model predictions from Béthermin et al. (2011), which are lower than the Poisson power levels found in this paper and lower than the 
Table 1. Summary of the 19 parameters describing the CIB, foregrounds and calibration that we vary in the likelihood analysis. All amplitude parameters have units of $(\mu \mathrm{K})^{2}$. Square brackets denote uniform prior ranges, while parentheses indicate the mean and standard deviation of Gaussian priors.

\begin{tabular}{|c|c|c|c|}
\hline Type & Parameter & Prior range & Definition \\
\hline CIB & $\begin{array}{l}A_{353}^{\mathrm{cib}} \\
A_{545}^{\mathrm{cib}} \\
A_{857}^{\mathrm{cib}} \\
r_{353}^{\mathrm{cib}} \times 545 \\
r_{353 \times 8}^{\mathrm{cib}} \times 857 \\
r_{545 \times 857}^{\mathrm{cib}} \\
\gamma^{\mathrm{cib}}\end{array}$ & $\begin{array}{c}{\left[10^{2}, 10^{5}\right]} \\
{\left[10^{4}, 10^{8}\right]} \\
{\left[10^{7}, 10^{10}\right]} \\
{[-0.5,1]} \\
{[-0.5,1]} \\
{[-0.5,1]} \\
{[0.0,1.5]}\end{array}$ & $\begin{array}{l}\text { Clustered CIB power at } \ell=2000 \text { (in } \mathcal{D}_{\ell} \text { ) at } 353 \mathrm{GHz} \\
\text { As for } A_{353}^{\text {cib }} \text { but at } 545 \mathrm{GHz} \\
\text { As for } A_{353}^{\text {cib }} \text { but at } 857 \mathrm{GHz} \\
\text { CIB correlation coefficient between } 353 \text { and } 545 \mathrm{GHz} \\
\text { As for } r_{353 \times 545}^{\text {cib }} \text { but between } 353 \text { and } 545 \mathrm{GHz} \\
\text { As for } r_{353 \times 545}^{\text {cib but between } 545 \text { and } 857 \mathrm{GHz}} \\
\text { Spectral index of the CIB angular power spectrum }\end{array}$ \\
\hline Dust & $\begin{array}{l}A_{353}^{\text {dust }} \\
A_{545}^{\text {dust }} \\
A_{857}^{\text {dust }}\end{array}$ & $\begin{array}{l}{\left[10^{2}, 10^{5}\right]} \\
{\left[10^{5}, 10^{8}\right]} \\
{\left[10^{8}, 10^{11}\right]}\end{array}$ & $\begin{array}{l}\text { Dust spectrum amplitude at } 353 \mathrm{GHz} \\
\text { As for } A_{353}^{\text {dust }} \text { but at } 545 \mathrm{GHz} \\
\text { As for } A_{353}^{\text {dust }} \text { but at } 857 \mathrm{GHz}\end{array}$ \\
\hline Poisson power & $\begin{array}{l}A_{353}^{\mathrm{ps}} \\
A_{545}^{\mathrm{ps}} \\
A_{857}^{\mathrm{ps}} \\
r_{353 \times 545}^{\mathrm{ps}} \\
r_{353 \times 857}^{\mathrm{ps}} \\
r_{545 \times 857}^{\mathrm{ps}}\end{array}$ & $\begin{array}{c}{\left[10^{2}, 10^{5}\right]} \\
{\left[10^{4}, 10^{7}\right]} \\
{\left[10^{5}, 10^{10}\right]} \\
{[-0.5,1]} \\
{[-0.5,1]} \\
{[-0.5,1]}\end{array}$ & $\begin{array}{l}\text { Poisson point source power at } \ell=2000\left(\text { in } \mathcal{D}_{\ell}\right) \text { at } 353 \mathrm{GHz} \\
\text { As for } A_{353}^{\mathrm{ps}} \text { but at } 545 \mathrm{GHz} \\
\text { As for } A_{353}^{\mathrm{ps}} \text { but at } 857 \mathrm{GHz} \\
\text { Poisson power correlation coefficient between } 353 \text { and } 545 \mathrm{GHz} \\
\text { As for } r_{353 \times 545}^{\mathrm{ps}} \text { but between } 353 \text { and } 545 \mathrm{GHz} \\
\text { As for } r_{353 \times 545}^{\mathrm{ps}} \text { but between } 545 \text { and } 857 \mathrm{GHz}\end{array}$ \\
\hline Calibration & $\begin{array}{l}\mathrm{cal}_{353} \\
\mathrm{cal}_{545} \\
\mathrm{cal}_{857}\end{array}$ & $\begin{array}{l}(1,0.0078) \\
(1,0.061) \\
(1,0.064)\end{array}$ & $\begin{array}{l}\text { Calibration factor at } 353 \mathrm{GHz} \\
\text { Calibration factor at } 545 \mathrm{GHz} \\
\text { Calibration factor at } 857 \mathrm{GHz}\end{array}$ \\
\hline
\end{tabular}

more recent model predictions of Béthermin et al. (2012b). In the 2013 Planck cosmological parameter analysis (Planck Collaboration XV 2014c), the clustered CIB was modelled as a power law with a Gaussian prior $\gamma^{\text {cib }}=0.7 \pm 0.2$ together with weak priors on the Poisson power levels. In the frequency range used for the CMB analysis, 100-217 GHz, consistently low values, $\gamma^{\text {cib }}=0.40$ \pm 0.15 , were found from the Planck spectra.

We can anticipate values for the amplitude of the clustered CIB power based on previous measurements. For example, using the measured spectra reported in table D.2 of P13 and subtracting their best-fitting Poisson powers, gives the following estimates:

$A_{353}^{\mathrm{cib}} \approx 2.2 \times 10^{3} \mu \mathrm{K}^{2}$,

$A_{545}^{\mathrm{cib}} \approx 4.2 \times 10^{5} \mu \mathrm{K}^{2}$,

$A_{857}^{\text {cib }} \approx 1.1 \times 10^{9} \mu \mathrm{K}^{2}$.

In our likelihood, we fit for these three amplitudes, plus three correlation coefficients that parametrize the cross-frequency spectra. For example, we take $A_{353 \times 545}^{\mathrm{cib}}=r_{353 \times 545}^{\mathrm{cib}} \sqrt{A_{353}^{\text {cib }} A_{545}^{\text {cib }}}$.

\subsubsection{Halo model template for the CIB}

We also consider an extended halo model, as in P13 that associates galaxies with dark matter haloes and sub-haloes, and adopts a specific parametric relation between galaxy luminosity and mass of the host sub-halo. In P13, such a model is fitted to auto and crossfrequency spectra from Planck and IRAS data in the frequency range 217-857 and $3000 \mathrm{GHz}$, respectively. The autofrequency spectra of the P13 halo model at 353, 545 and $857 \mathrm{GHz}$ are shown in Fig. 2. Here the spectra are normalized, arbitrarily, at $\ell=1500$. One can clearly see a transition from a two-halo term dominating at multipoles $\ell \lesssim 1000$ to a steeper behaviour at multipoles $\ell \gtrsim 2000$, where the one-halo term dominates. The dotted line in Fig. 2 shows a power law with an index $\gamma^{\text {cib }}=0.54$, close to the best-fitting spectral index found in our likelihood analysis. Evidently, over most of the multipole range $(500 \lesssim \ell \lesssim 2000)$, the

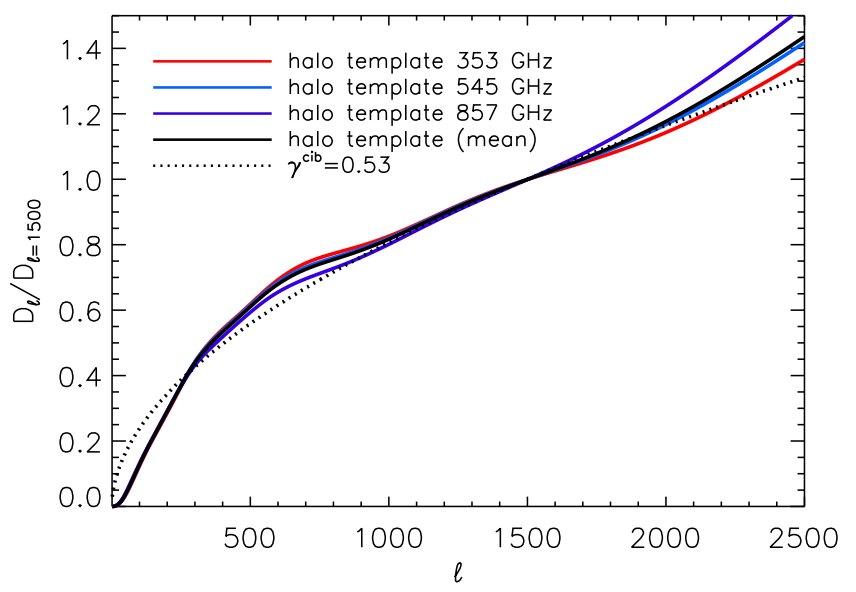

Figure 2. Halo model templates from P13 for the three autofrequency spectra at 353,545 and $857 \mathrm{GHz}$, normalized to unity at $\ell=1500$. The solid black line shows the fiducial halo model template shape adopted in this paper (which is similar to the $\mathrm{P} 13$ template at $545 \mathrm{GHz}$ ). The dotted line shows a power-law CIB spectrum with index $\gamma^{\text {cib }}=0.53$, the best-fitting index found in our analysis.

differences between these template shapes are small. In our analysis, we have therefore constructed a single halo model template shape from the mean of the three autospectra plotted in Fig. 2, which is shown as the solid black line in the figure. (This is actually quite close to the $545 \mathrm{GHz}$ halo model template.) We use this mean template in most of our analysis of the halo model, although in Section 5.4, we investigate the effect of changing to one of the halo model templates of Viero et al. (2013). In the rest of the paper, we refer to this template as 'halo model template' or 'P13 halo model'.

Our analysis of the halo model assumes the mean template shape plotted in Fig. 2, normalized to unity at $\ell=2000$. The clustered CIB contribution to the Planck spectra is then parametrized by three amplitudes $A_{v}^{\mathrm{cib}}$ and three correlation coefficients $r_{v \times \nu^{\prime}}^{\mathrm{cib}}$, as in our analysis of the power-law model. 


\subsection{CMB and thermal SZ}

The CMB power is significant at $353 \mathrm{GHz}$, but sub-dominant compared to all other components at 545 and $857 \mathrm{GHz}$ (see Fig. 8 below that shows the various components deduced from the likelihood analysis). In this paper, we do not fit for the CMB power spectrum since it is determined to high precision by the Planck data at lower frequencies. We therefore fix the CMB spectrum to that of the fiducial $\Lambda$ CDM cosmology described in Section 1. We do not include thermal SZ signal in our model, since its amplitude is negligible at frequencies $v \geq 353 \mathrm{GHz}$ compared to the CIB amplitude (see P13).

\subsection{Galactic dust}

Diffuse Galactic emission dominates over the CIB at low multipoles $(\ell \lesssim 500)$ even in the cleanest regions of the sky. We subtract Galactic emission in the power spectrum domain following the approach adopted in the Planck CMB analysis (Planck Collaboration XV 2014c; Planck Collaboration XI 2016b). The idea is to construct a power spectrum template for Galactic dust emission by differencing the spectra computed on different masks. The isotropic components, including CMB, CIB and extragalactic sources, should cancel in forming the difference leaving only a contribution from Galactic dust emission. Our main assumption is that the shape of the power spectrum of Galactic dust emission outside of a given mask can be approximated by a smooth fit to the mask-differenced power spectrum. This provides templates for the dust power spectra that are introduced into the likelihood analysis together with nuisance parameters to fix the amplitudes of the dust contribution at each frequency. ${ }^{6}$

We begin by investigating the 'universality' of Galactic dust emission, i.e. whether the shape of the dust power spectrum varies with frequency and/or sky area. Fig. 3 tests variations of the shape of the dust spectrum with sky coverage. We show the mask-differenced $545 \mathrm{GHz}$ spectra for each of the point source masks used in this paper. In each case, we have rescaled the amplitudes of the spectra to match the amplitude of the mask 40-mask 25 spectrum over the multipole range $100 \leq \ell \leq 300$. Up to mask 60 , the power spectra are almost independent of the point source mask and scale accurately independent of the sky coverage. We begin to see some departures from universality for mask 70 and mask 80 that are stronger when we apply the $857 \mathrm{GHz}$ point source mask compared to the $353 \mathrm{GHz}$ point source mask. The departures from universality over these large sky areas are caused mainly by the anisotropy of the point source masks (see Fig. 1) rather than by changes in the properties of the diffuse Galactic dust emission. Anisotropy in the point source masks arises, for example, from not masking sources in regions of strong Galactic cirrus. It induces statistical anisotropy in the unmasked sources, potentially leaving a non-zero contribution to the mask-differenced spectra in the mean. However, up to mask 60 , there is no evidence of any change in the shape of the dust power spectrum with sky area for any of the point source masks used in this paper. We find very similar behaviour for the 353 and $857 \mathrm{GHz}$ mask-differenced spectra.

Next, we test for variations in the shape of the dust power spectrum with frequency. Fig. 4 shows the 353, 545 and $857 \mathrm{GHz}$ maskdifferenced spectra for two mask sizes, mask 40-mask 25 and mask

\footnotetext{
${ }^{6}$ We note that even in analyses using $\mathrm{H}_{\mathrm{I}}$ as a tracer of dust emission (e.g. PEP and P13), some assumptions need to be made to extrapolate the Galactic dust spectrum to scales smaller than the resolution limit of the $\mathrm{H}_{\mathrm{I}}$ data.
}
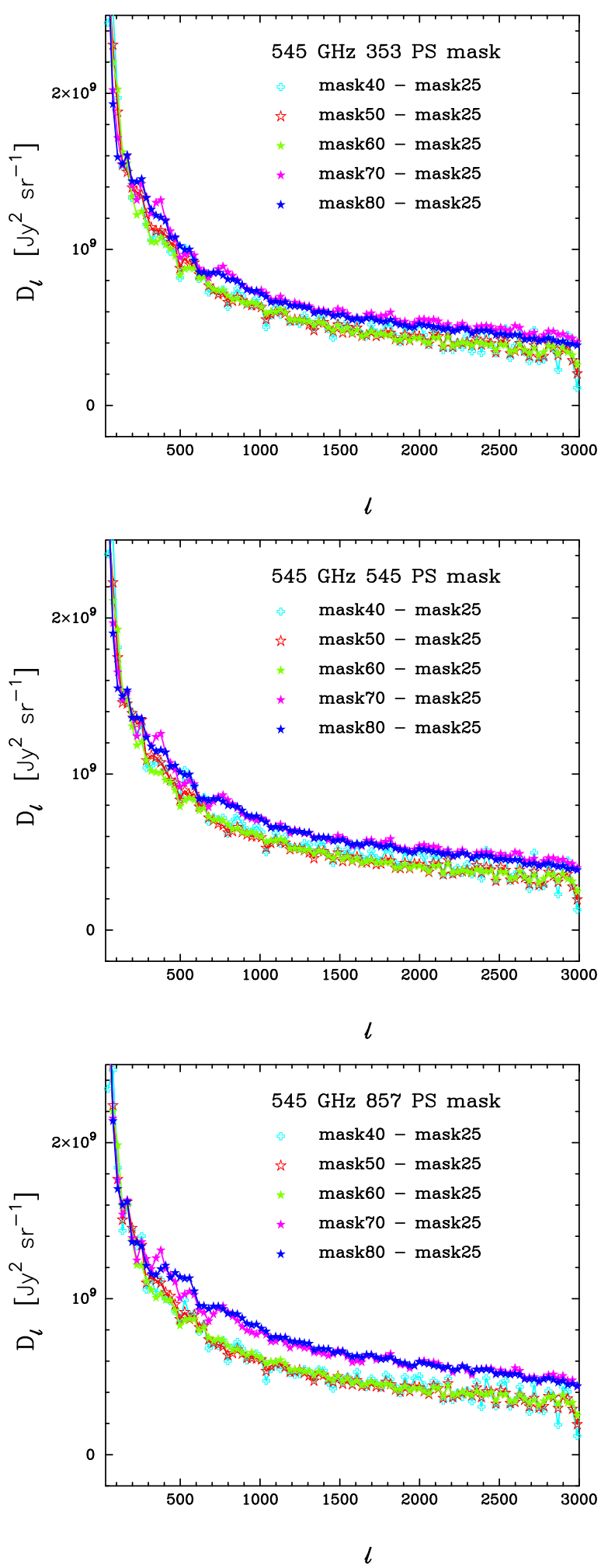

Figure 3. Mask-differenced power spectra at $545 \mathrm{GHz}$ for the three point source masks used in this paper. In each panel, the spectra have been rescaled to match the amplitude of the mask 40-mask 25 spectrum over the multipole range $100 \leq \ell \leq 300$. 


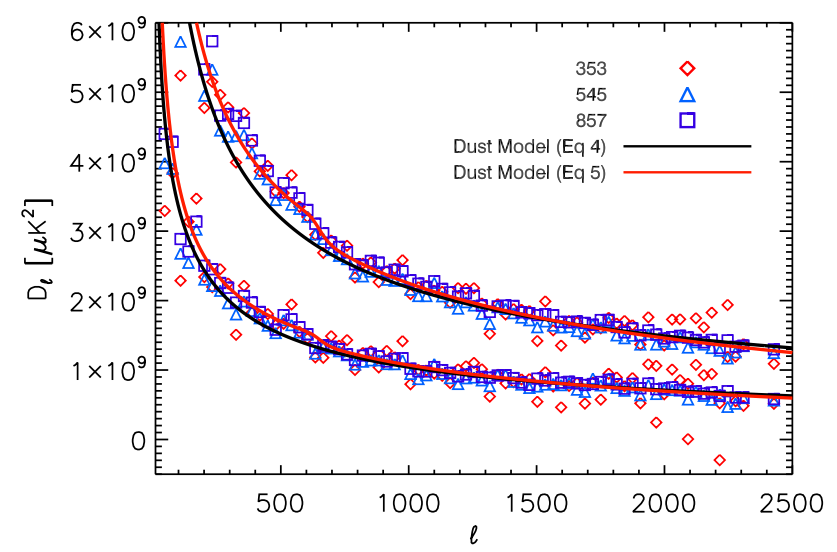

Figure 4. Mask-differenced power spectra at 353 (red diamonds), 545 (blue triangles) and at $857 \mathrm{GHz}$ (purple squares). The 353 and $545 \mathrm{GHz}$ spectra have been rescaled to match the amplitudes of the $857 \mathrm{GHz}$ spectra. Results are shown for the difference of masks 40 and 25 and masks 50 and 25, the latter having larger amplitude. The union point source masks are used for all of these estimates. Dust templates based on equations (4) and (5) are shown by the black and red solid lines, respectively.

50-mask 25, adopting the union point source mask. The 353 and $545 \mathrm{GHz}$ spectra have been rescaled to match the amplitude of the $857 \mathrm{GHz}$ spectrum. For each of the two masks shown here, the dust power spectra at each frequency are closely similar. In fact, the universality of the dust power spectrum as a function of frequency extends to larger sky areas, but for our purposes, the higher amplitude of Galactic dust emission and the associated higher cosmic variance at lower multipoles limits the useful area over which we can separate CIB and Galactic dust at low multipoles. For this reason, most of our results are based on likelihood fits to spectra computed over 50 per cent of the sky or less. Over this area of sky, the results shown in Figs 3 and 4 demonstrate that the Galactic dust power spectrum is accurately universal over sky area and frequency.

We assume further that the dust emission does not decorrelate across frequencies, i.e. we set $A_{v \times v^{\prime}}^{\text {dust }}=\left(A_{v}^{\text {dust }} A_{v^{\prime}}^{\text {dust }}\right)^{1 / 2}{ }^{7}$ Some variations in the spectral energy distribution (SED) of dust across the sky are expected from local variations in the dust temperature and frequency dependence of the opacity (due to variations in the dust grain properties). SED variations would lead to decorrelation across frequencies, with $C_{\ell}^{\text {dust, } v \times v^{\prime}} \neq\left(C_{\ell}^{\text {dust, } v \times v} C_{\ell}^{\text {dust, } v^{\prime} \times v^{\prime}}\right)^{1 / 2}$. We can test the degree of correlation across frequencies by forming a mask-differenced spectrum between different frequencies, e.g. $\hat{C}_{\ell}^{\nu \times v^{\prime}}(\operatorname{mask} 40)-\hat{C}_{\ell}^{\nu \times \nu^{\prime}}(\operatorname{mask} 25)$. The resulting correlation coefficient for $v=545 \mathrm{GHz}$ and $v^{\prime}=857 \mathrm{GHz}$ is shown in Fig. 5. This shows that any decorrelation is less than 1 per cent, and so we ignore it in our dust modelling. The high level of correlation shown in Fig. 5 is consistent with that expected given the SED variations inferred from analysis of Planck maps and the IRAS $100 \mu \mathrm{m}$ map in Planck Collaboration XI (2014b). This can be demonstrated by computing the mask-differenced correlation coefficients from the dust maps that form part of the Planck full focal plane simulations (Planck Collaboration XII 2016c). Full details of the construction of these dust maps are given in Planck Collaboration XII (2016c); see also Planck Collaboration XLVIII (2016e). Briefly, they are based on an estimate of the dust emission at $353 \mathrm{GHz}$, constructed with

\footnotetext{
${ }^{7}$ Unlike the Planck analysis of cosmological parameters (Planck Collaboration XI 2016b), in this paper, we always use the same Galactic mask for all frequencies.
}

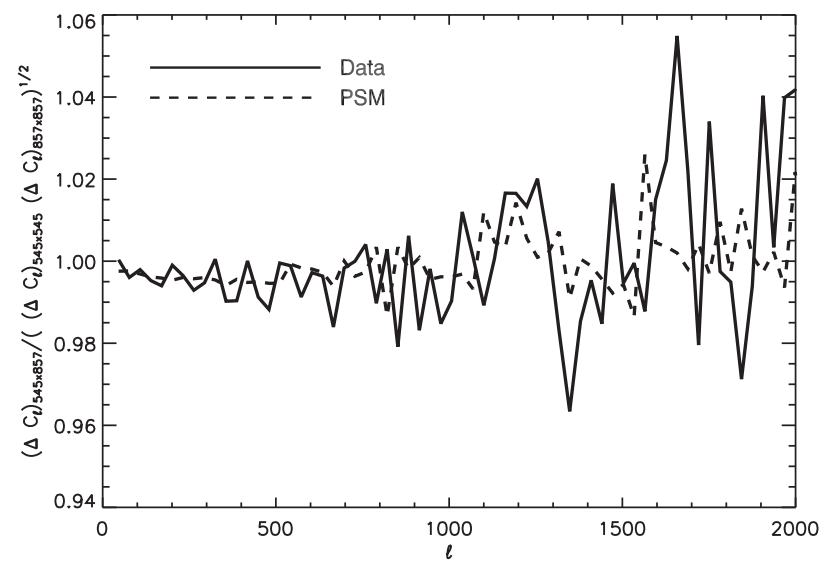

Figure 5. Measured correlation coefficient of the mask-differenced power spectrum for $545 \times 857$ between masks 40 and 25 (solid line). This is in good agreement with the correlation coefficient measured from the 545 and $857 \mathrm{GHz}$ dust maps in the Planck Sky Model (dashed lines). Dust is more than 99 per cent correlated between these frequencies.

the Generalized Needlet Internal Linear Combination method (e.g. Planck Collaboration XLVIII 2016e), which is scaled in frequency in each pixel as a modified blackbody with temperature and spectral index taken from Planck Collaboration XI (2014b). The measured correlation of these dust maps is shown in Fig. 5 and agrees well with our analysis of the 545 and $857 \mathrm{GHz}$ Planck maps.

In the Planck 2013 analysis of cosmological parameters, the following simple parameteric model for the dust power spectrum was adopted:

$\mathcal{D}_{\ell}^{\text {dust }, v \times v^{\prime}}=A_{v \times v^{\prime}}^{\text {dust }} \frac{(100 / \ell)^{\alpha}}{\left[1+\left(\ell / \ell_{c}\right)^{2}\right]^{\gamma / 2}}$.

The parameters $\alpha, \ell_{c}$ and $\gamma$ are fixed by matching to the maskdifferenced dust power spectra. Given the universality of the dust power spectrum, neither the choice of frequency nor mask sizes are critical in fixing these parameters. Using the $857 \mathrm{GHz}$ mask 40-mask 25 spectrum, we find $\alpha=0.387, \ell_{c}=162.9$ and $\gamma=0.168$, so that asymptotically $C_{\ell}^{\text {dust }} \propto \ell^{-2.55}$. This $\ell$ dependence at high multipoles is compatible with previous high-resolution measurements of the dust power spectrum in high Galactic latitude cirrus clouds, $C_{\ell} \propto \ell^{-2.7 \pm 0.1}$ (Miville-Deschênes, Lagache \& Puget 2002; Miville-Deschênes et al. 2010), although our mask-differenced results consistently give slopes at the shallower end of this range. For a given mask, the dust power in the $v \times v^{\prime}$ spectrum is specified by the parameter $A_{\nu \times v^{\prime}}^{\text {dust }}$. For the values of $\alpha, \ell_{c}$ and $\gamma$ given above, $A_{v \times v^{\prime}}^{\text {dust }}$ is the dust power at multipole $\ell=10$.

The solid black lines in Fig. 4 show the model of equation (4) compared to the mask 40-mask 25 and mask 50-mask 25 spectra for the 353,545 and $857 \mathrm{GHz}$ spectra. The model provides a reasonably good fit but one can see evidence for an excess at $\ell \approx 500$. We have therefore fit the dust spectra to a slightly more complicated fitting function:

$\mathcal{D}_{\ell}^{\text {dust,mask50 }} \propto\left(\frac{100}{\ell}\right)^{\alpha_{2}}+B\left(\frac{\ell}{1000}\right)^{\beta_{2}}\left[1+\left(\frac{\ell}{\ell_{c 2}}\right)^{\gamma_{2}}\right]^{\delta}$,

where $B, \alpha_{2}, \beta_{2}, \ell_{c 2}, \gamma_{2}$ and $\delta$ are parameters that we fit to the $857 \times 857$ mask $50-$ mask 25 spectrum. The best-fitting parameters are $B=0.74, \alpha_{2}=0.19, \beta=-0.58, \ell_{c 2}=543.1, \gamma_{2}=11.6$ and $\delta=0.07$. We normalize the template at $\ell=10$, so that the dust amplitudes $A_{v \times v^{\prime}}^{\text {dust }}$ derived using the templates of equations (5) and (4) can be compared directly. The best-fitting template of 
equation (5) is shown by the red lines in Fig. 4. Equation (5) provides a better fit to both the mask 40-mask 25 and mask 50-mask 25 spectra than equation (4), capturing the small excess at $\ell \approx 500$. Unless otherwise stated, we use the template shape of equation (5) in our baseline analysis.

In summary, using mask-differenced spectra to isolate the contribution from Galactic dust, we find that the dust power spectrum has a nearly universal shape independent of frequency and sky fraction up to mask 60 . For larger sky fractions, we see relatively small departures from universality that depends on the point source mask and are most likely associated with the anisotropy of the point source masks at low Galactic latitudes. The shape of the dust power spectrum is modelled accurately by the fitting function of equation (5), which provides a template shape used in our likelihood analysis. We have verified that the dust power spectra are almost perfectly correlated over the frequency range 353-857 GHz. We therefore solve for three dust amplitudes, $A_{353}^{\text {dust }}, A_{545}^{\text {dust }}$ and $A_{857}^{\text {dust }}$ in our likelihood analysis assuming that the dust power spectrum is completely correlated across frequencies.

\subsection{Poisson power from unmasked point sources}

The Poisson contribution to the power spectra arises from shot noise of extragalactic sources. P13 estimate that the Poisson power from radio sources is less than 2 percent of that from infrared sources at $v \geq 353 \mathrm{GHz}^{8}$ As a result, we consider here only the Poisson power from infrared sources. The Poisson contribution to the power spectrum is constant, $C_{\ell}^{\mathrm{ps}}=$ const. We model this contribution with a single amplitude parameter, giving $\mathcal{D}_{\ell}^{\mathrm{ps}}$ at multipole $\ell=2000$, for each auto-frequency spectrum $\left(A_{353}^{\mathrm{ps}}, A_{545}^{\mathrm{ps}}\right.$, and $\left.A_{857}^{\mathrm{ps}}\right)$ and a cross-correlation coefficient for each cross-frequency spectrum (e.g. $A_{353 \times 545}^{\mathrm{ps}}=r_{353 \times 545}^{\mathrm{ps}} \sqrt{A_{353}^{\mathrm{ps}} A_{545}^{\mathrm{ps}}}$ ). In the rest of this section, we summarize briefly the methodology that we have used to calculate the Poisson power levels from source counts. These calculations are not used directly in the main likelihood analysis; instead they serve as a rough consistency check of the Poisson power that we infer from the Planck spectra.

The Poisson power at frequency $v$ from sources below a fluxdensity limit is

$C_{\ell}^{\mathrm{ps}, v \times v}=\int_{0}^{S_{\nu, \mathrm{cut}}} S_{v}^{2} \frac{\mathrm{d} N}{\mathrm{~d} S_{v}} \mathrm{~d} S_{v}$,

where $\mathrm{d} N / \mathrm{d} S_{v}$ is the differential number count measured at frequency $v$ and $S_{v \text {, cut }}$ is an effective flux-density cut above which sources are masked. The Poisson power from infrared sources is dominated by sources well below the Planck flux-density cuts. We must therefore rely on theoretical models for $\mathrm{d} N / \mathrm{d} S_{v}$, or measurements of number counts from higher resolution experiments capable of detecting sources to lower flux densities. Here, we model the source count distribution by fitting to number counts determined from experiments at frequencies similar to Planck. For this purpose, we use number counts at 350 and $500 \mu \mathrm{m}$ from Herschel (Clements et al. 2010; Glenn et al. 2010; Oliver et al. 2010; Béthermin et al. 2012a) and at $850 \mu \mathrm{m}$ from the Submillimeter Common-User Bolometer Array 2 (SCUBA2) Cosmology Legacy Survey (S2CLS; Geach et al. 2017). We also plot source count measurements at $870 \mu \mathrm{m}$ : Atacama Pathfinder Experiment Telescope (APEX)/Large Apex Bolometer Camera (LABOCA) obser-

\footnotetext{
${ }^{8}$ Note that the effective flux-density cuts for point source masks at $353 \mathrm{GHz}$ used in P13 and in this paper are quite similar (around $315 \mathrm{mJy}$ for P13 compared to 400 mJy here).
}

vations (Weiß et al. 2009) of the Extended Chandra Deep Field South (ECDF); SCUBA (Coppin et al. 2006; Simpson et al. 2015); and Atacama Large Millimeter/submillimeter Array (ALMA) pointings of the ECDF LABOCA field (Karim et al. 2013). Since these are based on smaller areas of sky, and are not in agreement with each other, we use only the larger catalogue from S2CLS (together with Planck counts) to predict the Poisson power level. We comment on the differences in the number counts from different surveys around $850 \mu \mathrm{m}$ in Section 5.5. At higher flux density, we use the source counts from Planck Collaboration VII (2013). The details of the individual data sets, (including colour-corrections to the Planck bands), source count model and our fitting procedure are described in Appendix B. The source counts are plotted in Fig. 6 (see also fig. 10 of Planck Collaboration VII 2013, for an earlier version of a similar figure).

Briefly, we fit a simple model consisting of a double power-law term and a constant term to the observed counts $S^{2.5} \mathrm{~d} N / \mathrm{d} S$ using MCMC, ${ }^{9}$ propagating the uncertainties in the model parameters to our predicted Poisson power levels for each frequency band. Our fits to the counts are shown by the purple lines and grey bands in Fig. 6 and provide good fits to the data, except at $850 \mu \mathrm{m}$ where the data are discrepant. This discrepancy is discussed in more detail in Appendix B. The solid black lines in Fig. 6 show number counts predicted from the theoretical models of Béthermin et al. (2012b, hereafter B12); see also Béthermin et al. (2012a). Qualitatively, the B12 model matches the source counts at 500 and $350 \mu \mathrm{m}$, but not at $870 \mu \mathrm{m}$, where the model predicts more faint sources at flux densities below $10 \mathrm{mJy}$ than reported by Weiß et al. (2009) and Karim et al. (2013). The B12 model is in agreement with the new counts of Geach et al. (2017) at the faint flux densities, but predicts more bright sources at flux densities greater than $10 \mathrm{mJy}$.

From our MCMC fits to the number counts, the integrals of equation (6) give the following amplitudes and $1 \sigma$ statistical errors:

$A_{353}^{\mathrm{ps}}=(1.4 \pm 0.1) \times 10^{3} \mu \mathrm{K}^{2}$,

$A_{545}^{\mathrm{ps}}=(3.7 \pm 0.2) \times 10^{5} \mu \mathrm{K}^{2}$,

$A_{857}^{\mathrm{ps}}=(9.0 \pm 0.3) \times 10^{8} \mu \mathrm{K}^{2}$.

These numbers are computed using the nominal flux-density limits appropriate to our point source masks at each frequency as the upper limits in equation (6). However, since the point source amplitudes are dominated by faint sources the predictions of equation (7) are rather insensitive to the upper limits; for example, doubling the upper limits increases the predicted amplitude at $353 \mathrm{GHz}$ by 14 per cent and the 545 and $857 \mathrm{GHz}$ amplitudes by a few per cent. The statistical errors quoted in equation (7) almost certainly underestimate the true errors given the poor quality of the fits at 350 and $850 \mu \mathrm{m}$. This should be borne in mind when comparing the predictions in equation (7) with the Poisson power levels deduced from our likelihood analysis.

For reference, the predictions for the B12 model (from table 6 of P13) are $2.1 \times 10^{3}, 3.1 \times 10^{5}$ and $6.9 \times 10^{8} \mu \mathrm{K}^{2}$ at 353,545 and $857 \mathrm{GHz}$, respectively. We note that the model predictions that we quote here have been colour-corrected from a CIB SED to the photometric convention $\nu I_{v}=$ constant. Generally, the modelled $C_{\ell}^{\mathrm{ps}, v \times v^{\prime}}$ has to be multiplied by a factor $c c_{v} \times c c_{v^{\prime}}$ in comparing to the data, where for the colour-corrections $c c_{v}$, we adopt the values in P13: 1.097, 1.068 and 0.995 at 353, 545 and $857 \mathrm{GHz}$, respectively.

\footnotetext{
${ }^{9}$ We use emcee, an open-source PYTHON package (Foreman-Mackey et al. 2013; http://dan.iel.fm/emcee/current/) to perform the MCMC.
} 

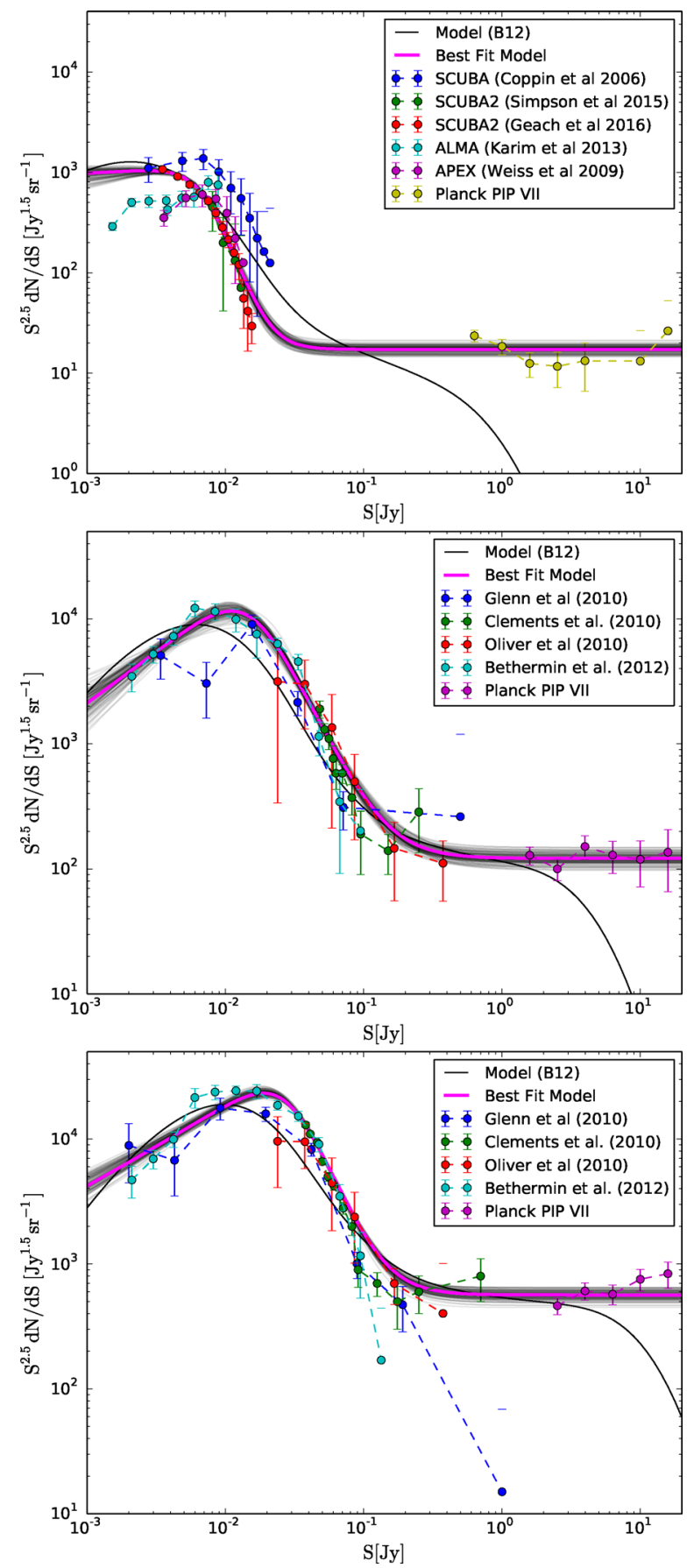

Figure 6. Differential number counts from measurements with Planck (Planck Collaboration VII 2013), Herschel (Clements et al. 2010; Glenn et al. 2010; Oliver et al. 2010; Béthermin et al. 2012a), and APEX, SCUBA and ALMA (Coppin et al. 2006; Weiß et al. 2009; Simpson et al. 2015; Geach et al. 2017) at 870 or 850 (345 GHz; top), 500 (600 GHz; middle) and $350 \mu \mathrm{m}(857 \mathrm{GHz}$; bottom). Also shown (thick purple lines) are the bestfitting double power-law models (together with the models from individual MCMC chains in grey to give some indication of the statistical uncertainty in the model) and the B12 model prediction (thin black lines). The break in the B12 model at high flux densities comes from the minimum redshift considered in the model $\left(z=10^{-3}\right)$. Note that for $870 \mu \mathrm{m}$, the data sets from Coppin et al. (2006), Weiß et al. (2009) and Simpson et al. (2015) are shown here for illustration only and are not used in our fits (see Appendix B). The Planck counts have been colour-corrected to match the central frequencies of the external data.

\section{CIB LIKELIHOOD}

We construct a likelihood using the CAMSPEC software, following closely the approach described for the Planck CMB analysis in Planck Collaboration XV (2014c). We form the six beam- and maskdeconvolved power spectra, $\hat{C}_{\ell}^{v \times v^{\prime}}$, in the multipole range $50 \leq \ell \leq$ 2500 , by cross-correlating the yearly maps. Although Galactic dust dominates the lowest multipoles for all spectra, except for $353 \times 353$ where the $\mathrm{CMB}$ is dominant, we retain multipoles down to $\ell=50$. As we shall see below, the low amplitude of the CIB compared to the dust power spectrum at multipoles $\ell \lesssim 300$, together with cosmic variance in the dust, means that it is difficult to separate these components accurately at low multipoles.

We adopt a Gaussian likelihood for the $\hat{C}_{\ell}^{v \times v^{\prime}}$, and include all correlations between the six cross-spectra in the covariance matrix. The covariance matrix is calculated assuming a fiducial model and includes sample variance from the CMB, Galactic dust, CIB and also the Planck anisotropic instrument noise, using the analytic expressions in Efstathiou (2004); see Appendix A for a summary. We compare the measured spectra with the theoretical model predictions as in equation (1). In doing so, the theoretical model is divided by the product of the appropriate map-level calibration parameters, $c a l_{v} \times c a l_{v^{\prime}}$. The parameters used in our likelihood analysis and their prior ranges are summarized in Table 1.

In constructing the covariance matrix for the power spectra, we approximate the CIB as Gaussian random fields, with total power spectra given by the sum of the clustered and Poisson powers. We also treat the dust as a statistically isotropic, Gaussian random field, i.e. for a given mask, the sample variance is constructed from a power spectrum with amplitude appropriate to the mask. These assumptions for dust are clearly incorrect (Miville-Deschênes et al. 2007). Much of the power comes from regions close to the edge of the Galactic mask, so the effective number of modes that contribute to the sample variance for a given mask is lower than if the fields were statistically isotropic. This will increase both the variance of the measured power spectra and also their covariance. In Section 6.1 and Appendix C, we develop a heuristic model that describes the dust emission as a Gaussian random field subject to a large-scale, anisotropic modulation. The sample variance in this model can be calculated straightforwardly. We show in Section 6.1 that the corrections from statistical anisotropy in this heuristic model have only a minor impact on our estimation of CIB model parameters, although they do improve the goodness of fit of the highfrequency spectra considerably.

Further discussion of the construction and validation of the likelihood can be found in Appendix A.

\subsection{Expected parameter errors and degeneracies}

Before presenting the results of the likelihood analysis, it is instructive to consider the expected errors on parameters and their covariances using a Fisher matrix analysis. We shall see that our likelihood results using Planck data, presented in Section 5, agree well with the forecasts summarized in this section.

For our assumed Gaussian likelihood, with a fiducial covariance matrix, the Fisher matrix has elements

$F_{i j}=\sum_{N, N^{\prime}} \sum_{\ell, \ell^{\prime}}^{\ell_{\max }} \frac{\partial C_{\ell}^{\mathrm{th}, N}}{\partial p_{i}} \operatorname{Cov}_{(\ell N)\left(\ell^{\prime} N^{\prime}\right)}^{-1} \frac{\partial C_{\ell}^{\mathrm{th}, N^{\prime}}}{\partial p_{j}}$,

where $p_{i}$ are the model parameters, $C_{\ell}^{\text {th, } N}$ is the theoretical power spectra from equation $(1), \operatorname{Cov}_{(\ell N)\left(\ell^{\prime} N^{\prime}\right)}$ is the covariance matrix of 
Table 2. Forecasted $1 \sigma$ errors on the CIB (power-law model), dust and Poisson power levels for mask 40. The calibration parameters are fixed to unity in this Fisher analysis. The forecasted errors are shown assuming different prior information on the levels of Poisson power: no prior (fourth column); Gaussian priors with a 20 per cent relative error in $A_{v}^{\mathrm{ps}}$ (fifth column); and $A_{v}^{\mathrm{ps}}$ fixed at their fiducial values (sixth column). All amplitude parameters have units of $\mu \mathrm{K}^{2}$.

\begin{tabular}{|c|c|c|c|c|c|}
\hline \multirow[t]{2}{*}{ Types } & \multirow[t]{2}{*}{ Parameter } & \multirow[t]{2}{*}{ Fiducial value } & \multicolumn{2}{|c|}{$1 \sigma$ forecasted error } & \multirow[b]{2}{*}{ Fixed } \\
\hline & & & No prior & 20 per cent prior & \\
\hline \multirow[t]{7}{*}{ CIB } & $A_{353}^{\text {cib }}$ & $2.2 \times 10^{3}$ & $8.7 \times 10^{1}$ & $8.3 \times 10^{1}$ & $3.2 \times 10^{1}$ \\
\hline & $A_{545}^{\mathrm{cib}}$ & $4.1 \times 10^{5}$ & $1.2 \times 10^{4}$ & $1.2 \times 10^{4}$ & $3.4 \times 10^{3}$ \\
\hline & $A_{857}^{545}$ & $8.4 \times 10^{8}$ & $3.3 \times 10^{7}$ & $3.2 \times 10^{7}$ & $1.1 \times 10^{7}$ \\
\hline & $r_{353 \times 545}^{\mathrm{cib}} 857$ & 0.95 & 0.01 & 0.01 & 0.01 \\
\hline & $r_{353 \times 857}^{\text {cib }}$ & 0.89 & 0.01 & 0.01 & 0.01 \\
\hline & $r_{545 \times 857}^{\mathrm{cib}}$ & 0.95 & $<0.01$ & $<0.01$ & $<0.01$ \\
\hline & $\gamma^{\mathrm{cib}}$ & 0.50 & 0.04 & 0.04 & 0.02 \\
\hline \multirow[t]{3}{*}{ Dust } & $A_{353}^{\text {dust }}$ & $3.3 \times 10^{3}$ & $1.3 \times 10^{2}$ & $1.3 \times 10^{2}$ & $1.0 \times 10^{2}$ \\
\hline & $A_{545}^{\text {dust }}$ & $8.9 \times 10^{5}$ & $2.1 \times 10^{4}$ & $2.1 \times 10^{4}$ & $1.6 \times 10^{4}$ \\
\hline & $A_{857}^{\text {dust }}$ & $5.1 \times 10^{9}$ & $7.0 \times 10^{7}$ & $7.0 \times 10^{7}$ & $4.4 \times 10^{7}$ \\
\hline \multirow[t]{6}{*}{ PS } & $A_{353}^{\mathrm{ps}}$ & $2.3 \times 10^{3}$ & $1.0 \times 10^{2}$ & $9.7 \times 10^{1}$ & - \\
\hline & $A_{545}^{\mathrm{PS}^{3}}$ & $3.6 \times 10^{5}$ & $1.4 \times 10^{4}$ & $1.4 \times 10^{4}$ & - \\
\hline & $A_{857}^{345}$ & $8.4 \times 10^{8}$ & $2.9 \times 10^{7}$ & $2.8 \times 10^{7}$ & - \\
\hline & $r_{353 \times 545}^{\mathrm{ps}}$ & 0.96 & 0.01 & 0.01 & 0.01 \\
\hline & $r_{353 \times 857}^{\mathrm{ps}}$ & 0.84 & 0.02 & 0.02 & 0.01 \\
\hline & $r_{545 \times 857}^{\mathrm{ps} \times 0.7}$ & 0.95 & $<0.01$ & $<0.01$ & $<0.01$ \\
\hline
\end{tabular}

the spectra and the sums over $N$ and $N^{\prime}$ are over the six distinct frequency combinations $v \times v^{\prime}$. The partial derivatives are calculated at the fiducial values of the parameters. Since the parametric model is very simple, the derivatives can all be computed analytically. The fiducial values that we adopt for the model parameters are given in Table 2; they are chosen to be close to the best-fitting values from our likelihood analysis for mask 40. For this Fisher analysis, we shall ignore the uncertainty in the absolute calibrations by fixing the calibration factors to unity. The calibration factors are totally degenerate with the dust, CIB, and Poisson amplitudes.

As an example of the results of the Fisher analysis, the forecasted (marginal) parameter errors are shown for mask 40 and the powerlaw CIB model in Table 2. We find that the amplitude parameters can be constrained to about 4 per cent precision or better, the correlation coefficients for the CIB and Poisson powers to better than 2 per cent and the power-law index of the CIB spectra to around 8 percent precision.

The correlation matrix of the 16 model parameters is shown in the middle panel of Fig. 7 and is very similar to what we estimate from the MCMC samples in the full likelihood analysis (top panel, which includes marginalisation over the calibration parameters). There are strong degeneracies amongst some parameters. For example, the dust amplitudes are positively correlated across frequencies, as are the CIB and Poisson amplitudes. This behaviour arises because over the frequency range that we analyse, Galactic emission is nearly perfectly correlated across frequencies and the CIB emission is also strongly correlated. Sample variance fluctuations in the CIB, for example, affect all frequency spectra similarly, positively correlating the errors on the CIB amplitude parameters across frequencies. A further significant degeneracy exists between the CIB amplitudes and the Poisson amplitudes at each frequency. As expected, these are strongly anticorrelated because their power spectra have similar shapes. We note also the CIB power-law index, $\gamma^{\text {cib }}$, is positively correlated with the CIB amplitudes. This is a consequence of our choice of the pivot scale at $\ell=2000$. For Planck, most of the statistical weight on the amplitude and shape of the clustered CIB spectrum comes from multipoles smaller than this, for which an increase in $\gamma^{\text {cib }}$ can be compensated by an increase in the amplitude. Similarly, $\gamma^{\text {cib }}$ is positively correlated with the dust amplitudes, which gets most weight from low multipoles, but is anticorrelated with the Poisson amplitudes, which get most weight from $\ell>2000$.

\subsubsection{Effect of priors on levels of Poisson power}

Separating the clustered CIB from the Poisson power is difficult. With Planck, we are able to measure the power spectra at multipoles $\ell \lesssim 3000$, where theoretical models predict clustered spectra that are shallower than Poisson (see Fig. 2). Power spectrum measurements at higher multipoles do not necessarily eliminate the degeneracy because in physical models the power spectrum of the clustered CIB steepens at non-linear scales, exacerbating the degeneracy with the Poisson power. Discriminating between a clustered and Poisson contribution, purely based on shape information of the power spectra, is therefore sensitive to both the multipole range and the assumed shapes of the clustered CIB spectra.

An alternative is to impose priors on the Poisson power estimated from source counts (Section 3.4), which can be obtained from high-resolution, deep observations over limited areas of the sky. This approach is also not straightforward, since it requires accurate number counts, with accurate error estimates (including sample variance) extending to low flux-density levels. To assess the impact of constraining the Poisson amplitudes, we augment our Fisher analysis with the following priors on the $A_{v}^{\mathrm{ps}}$ : Gaussian priors with relative errors of 20 per cent of the fiducial value; ${ }^{10}$ and very narrow priors that fix the Poisson levels completely. In both cases, we do not apply any priors on the Poisson correlation coefficients, $r_{v \times v^{\prime}}^{\mathrm{ps}}$. These examples are meant to be illustrative. The formal errors on the point source amplitudes predicted from number counts given in equation (7) are significantly smaller than 20 per cent. However,

\footnotetext{
${ }^{10}$ Note that in analysis of P13, priors on the Poisson power levels were imposed based on the B12 models, with estimated errors of 20 per cent.
} 

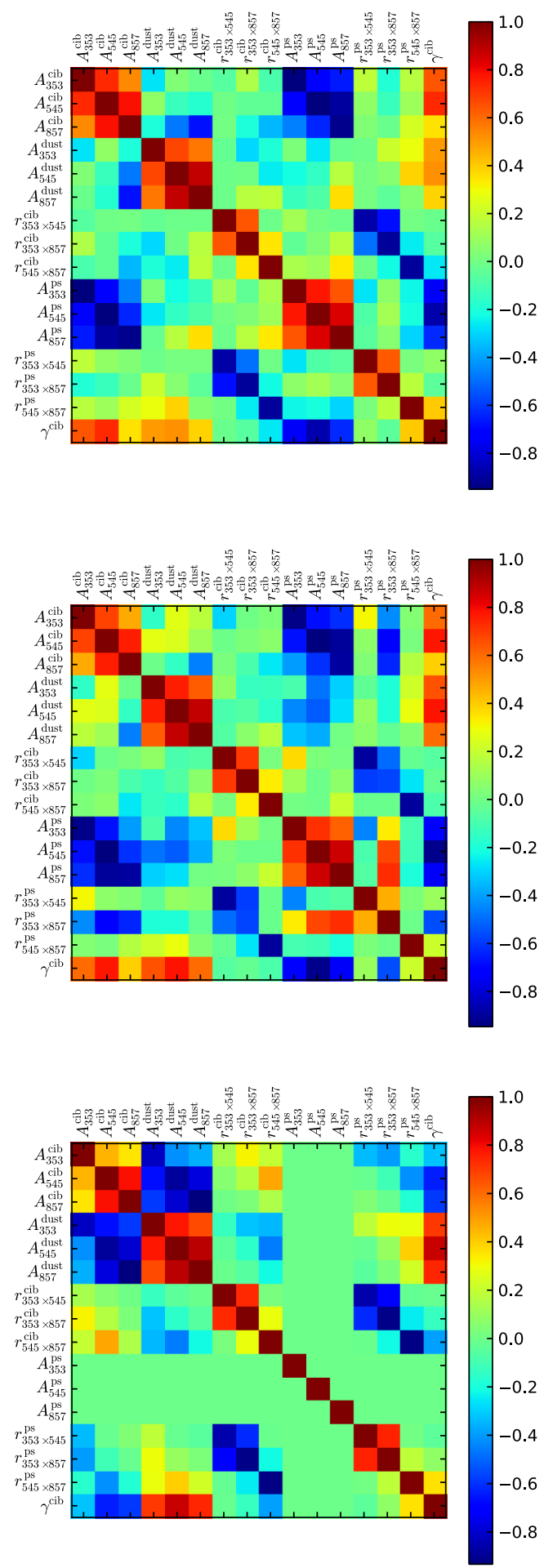

Figure 7. Correlation matrix for the 16 parameters describing the CIB (power-law model), dust and Poisson power levels for mask 40. The top panel shows the correlations estimated from MCMC samples of the likelihood for the Planck data, marginalizing over the calibration parameters. The middle plot shows the correlations calculated from the Fisher matrix, for fixed calibration parameters, and is very similar to what we find from the likelihood analysis. The bottom panel shows the effect of fixing the Poisson power levels in the Fisher matrix analysis. as mentioned in Secttion 3.4, these formal errors may well underestimate the true errors. (We shall see later that there is a large discrepancy at $353 \mathrm{GHz}$ in the Poisson power levels measured from Planck and those predicted by the counts of Weiß et al. 2009 and Karim et al. 2013.)

Our results with these priors are presented in Table 2 and Fig. 7. With the Poisson power levels constrained at the 20 per cent level, the parameter constraints and correlation properties are hardly changed (We do not show the associated correlation matrix in Fig. 7 for this reason.) However, when we fix the Poisson amplitudes, the uncertainties on both the CIB and dust amplitudes improve by at least a factor of 2 and similarly for the power-law index $\gamma^{\text {cib }}$. There are also some changes in the correlations between parameters. For example, the CIB and dust amplitudes are markedly anticorrelated, whereas there is no significant correlation without the tight priors on the $A_{v}^{\mathrm{ps}}$. Moreover, the amplitude and power-law index of the CIB switch from being positively correlated to anticorrelated. This is because the statistical weight in the determination of the amplitude and shape of the clustered CIB shifts to smaller scales when the Poisson power levels are fixed.

These results suggest that imposing priors on the Poisson power levels derived from source counts in our analysis of the Planck data has little impact unless the source count predictions are accurate to much better than 20 per cent. Even then, significant degeneracies between parameters remain.

\section{ANGULAR POWER SPECTRA AND PARAMETER RESULTS FROM PLANCK}

\subsection{Power spectra}

Fig. 8 shows the six auto and cross-frequency angular power spectra that we measure from cross-correlating the yearly maps for mask 40 (shown in Fig. 1). (The beam and mask are deconvolved from the spectra, which are binned assuming flat bandpowers in $\mathcal{D}_{\ell}$.) The figure also shows the best-fitting model for these spectra obtained from the full likelihood analysis assuming the power-law model for the clustered CIB, the decomposition of this best-fitting model into individual components and the residuals with respect to the best-fitting model.

The power spectra at 545 and $857 \mathrm{GHz}$ are signal dominated for multipoles $\ell<2000$, while the $353 \mathrm{GHz}$ spectrum is signal dominated only at $\ell<1000$. On large and intermediate scales, the $353 \mathrm{GHz}$ spectrum is dominated by dust and CMB, while at $857 \mathrm{GHz}$, dust is the dominant component for $\ell<1500$. At $545 \mathrm{GHz}$, the clustered CIB is the dominant signal over the multipole range $1000<\ell<2000$.

On the full likelihood, the power-law CIB model appears to fit the data well. For the fiducial sky fraction of 40 per cent, the best-fitting model has a reduced $\hat{\chi}^{2}=0.985$ for 14687 degrees of freedom (corresponding to all unbinned auto and cross-frequency spectra in the multipole range $\ell=50-2500$ ) that is acceptable to within $1.25 \sigma$ (although see below for a discussion of the goodness of fit of the binned spectra). We recover calibration constraints, $\mathrm{cal}_{v}$, that are close to unity and are consistent with our priors. As mentioned in Section 2, we have used conservative priors and have not imposed any priors on the relative calibration. The central values that we find for $\mathrm{Cal}_{545}$ from our likelihood analyses are somewhat larger than the more precise determinations reported in Planck Collaboration XLVI (2016d), which are based on Solar dipole and first acoustic peak calibrations, but our posterior distributions for $\mathrm{cal}_{545}$ are still consistent with the dipole-based calibrations given the size 

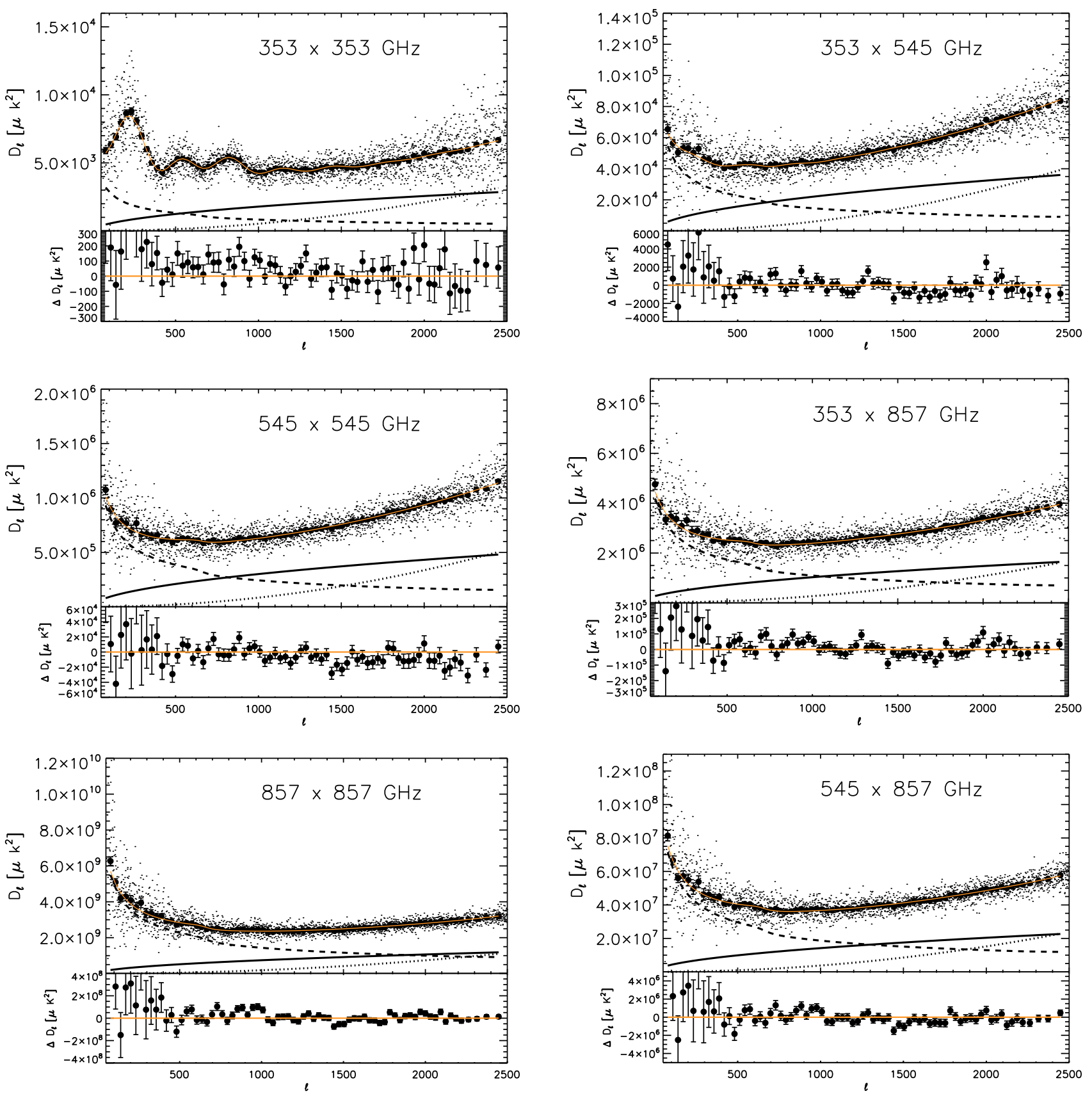

Figure 8. Measured angular power spectra for mask 40, constructed from cross-correlating Year-1 and Year-2 maps. The spectra are binned in flat bandpowers (for $\mathcal{D}_{\ell}$ ) with $\Delta \ell=31$. The lines show the best-fitting parametric models (based on the power-law CIB model): total (orange solid lines); clustered CIB (black solid lines); Galactic dust (black dashed lines); and Poisson power from the shot noise of extragalactic sources (black dotted lines). The error bars on the binned spectra are $\pm 1 \sigma$ errors determined from the diagonal components of the (binned) analytic covariance matrices. In each plot, the lower panels show the residuals of the binned spectra from the best-fitting model. The small dots in the upper panels show the power-spectra multipole-by-multipole.

of our errors. Our main parameter results are given in Table 3 and the best-fitting models are compared with the measured spectra in Figs 8 and 9 for the power-law and halo model CIB templates, respectively. Goodness-of-fit tests of individual auto- and crossfrequency spectra with respect to the best-fitting models of the full likelihood analysis are reported in Table 4.

In the rest of this section, we discuss various aspects of these results. Section 5.2 discusses the results for the clustered CIB for both the power-law model and the P13-based halo-model template. Section 5.3 describes the results of a 'joint-mask' analysis that further leverages the statistical anisotropy of the Galactic dust emis- sion. Our results are compared with previous measurements from Planck and Herschel in Section 5.4 and, in Section 5.5, we compare the Poisson amplitudes inferred from our spectra with expectations based on source counts. Appendix D discusses additional tests of the stability of our results to various data cuts and methodological changes.

\subsection{CIB models}

Using the power-law model for the clustered CIB power, we find (consistently across the three frequencies and sky fractions) that the 
Table 3. Best-fitting parameter values and 68 per cent marginalized errors from the full likelihood analysis. Results are shown for the power-law CIB model for masks 30, 40 and 50, and for the halo model template for mask 40 only (last column). We also include the results of a joint-mask analysis of the power-law model that uses spectra for masks 40 and 50. The number of degrees of freedom is 14687 for the power-law model, 14688 for the template model and 29390 for the joint-mask analysis. The frequency correlation coefficients $r^{\mathrm{cib}}$ and $r^{\mathrm{ps}}$ are omitted here; they are presented in Table 5. We also list the reduced $\chi^{2}$ (denoted $\hat{\chi}^{2}$ ) for each fit.

\begin{tabular}{|c|c|c|c|c|c|c|}
\hline \multirow[t]{3}{*}{ Type } & \multirow{3}{*}{$\begin{array}{l}\text { Parameter } \\
\hat{\chi}^{2}\end{array}$} & \multicolumn{5}{|c|}{ Best fit \pm 68 per cent error } \\
\hline & & mask 30 & mask 40 & mask 50 & Joint $^{\text {mask } 40+50}$ & mask 40 (halo model template) \\
\hline & & 1.005 & 0.985 & 0.977 & 1.024 & 0.994 \\
\hline \multirow[t]{4}{*}{ CIB } & $A_{353}^{\mathrm{cib}}\left(\times 10^{3}\right)$ & $2.50 \pm 0.07$ & $2.56 \pm 0.07$ & $2.42 \pm 0.07$ & $2.57 \pm 0.07$ & $2.63 \pm 0.06$ \\
\hline & $A_{545}^{\mathrm{cib}}\left(\times 10^{5}\right)$ & $4.50 \pm 0.20$ & $4.47 \pm 0.21$ & $4.25 \pm 0.21$ & $4.69 \pm 0.18$ & $4.74 \pm 0.22$ \\
\hline & $A_{857}^{\mathrm{cib}}\left(\times 10^{9}\right)$ & $1.09 \pm 0.07$ & $1.09 \pm 0.07$ & $1.00 \pm 0.07$ & $1.11 \pm 0.07$ & $1.22 \pm 0.08$ \\
\hline & $\gamma^{\mathrm{cib}}$ & $0.51 \pm 0.02$ & $0.53 \pm 0.02$ & $0.54 \pm 0.03$ & $0.56 \pm 0.02$ & - \\
\hline \multirow[t]{3}{*}{ Dust } & $A_{353}^{\text {dust }}\left(\times 10^{3}\right)$ & $4.31 \pm 0.19$ & $8.76 \pm 0.22$ & $16.44 \pm 0.29$ & $8.99 \pm 0.21,16.15 \pm 0.22$ & $8.14 \pm 0.22$ \\
\hline & $A_{545}^{\text {dust }}\left(\times 10^{6}\right)$ & $1.28 \pm 0.06$ & $2.61 \pm 0.11$ & $4.96 \pm 0.21$ & $2.77 \pm 0.10,5.00 \pm 0.18$ & $2.50 \pm 0.11$ \\
\hline & $A_{857}^{\text {dust }}\left(\times 10^{9}\right)$ & $6.85 \pm 0.45$ & $15.08 \pm 0.97$ & $28.48 \pm 1.80$ & $15.19 \pm 0.96,27.99 \pm 1.74$ & $14.45 \pm 0.91$ \\
\hline \multirow[t]{3}{*}{ PS } & $A_{353}^{\mathrm{ps}}\left(\times 10^{3}\right)$ & $2.15 \pm 0.07$ & $2.10 \pm 0.07$ & $2.25 \pm 0.07$ & $2.10 \pm 0.06$ & $2.06 \pm 0.05$ \\
\hline & $A_{545}^{\mathrm{p}}\left(\times 10^{5}\right)$ & $3.54 \pm 0.19$ & $3.42 \pm 0.20$ & $3.64 \pm 0.20$ & $3.50 \pm 0.18$ & $3.33 \pm 0.15$ \\
\hline & $A_{857}^{\mathrm{ps}}\left(\times 10^{8}\right)$ & $7.20 \pm 0.51$ & $7.34 \pm 0.53$ & $7.76 \pm 0.56$ & $7.20 \pm 0.51$ & $6.37 \pm 0.45$ \\
\hline \multirow[t]{3}{*}{ Cal } & $\mathrm{cal}_{353}$ & $1.00 \pm 0.01$ & $1.00 \pm 0.01$ & $1.00 \pm 0.01$ & $1.00 \pm 0.01$ & $1.00 \pm 0.01$ \\
\hline & $\mathrm{cal}_{545}$ & $1.05 \pm 0.04$ & $1.03 \pm 0.04$ & $1.04 \pm 0.04$ & $1.07 \pm 0.04$ & $1.05 \pm 0.04$ \\
\hline & cal $_{857}$ & $1.01 \pm 0.06$ & $1.01 \pm 0.06$ & $1.01 \pm 0.06$ & $1.02 \pm 0.06$ & $1.02 \pm 0.06$ \\
\hline
\end{tabular}

clustering component contributes about 54 per cent of the total CIB power at $\ell=2000$. We obtain a tight constraint on the CIB slope of $\gamma^{\mathrm{cib}}=0.53 \pm 0.02$. This value is consistent with that obtained in the 2013 analysis of cosmological parameters from Planck data alone (Planck Collaboration XV 2014c), $\gamma^{\text {cib }}=0.40 \pm 0.15$, using the 100,143 and $217 \mathrm{GHz}$ channels. Both analyses probe the clustered CIB over a similar range of multipoles. However, at frequencies $v$ $\leq 217 \mathrm{GHz}$, the high amplitude of the CMB and other foreground components, in addition to dust emission, makes it difficult to extract information on the CIB at multipoles $\ell \lesssim 1000$.

Although the best-fitting models gives acceptable $\chi^{2}$ using the full likelihood multipole by multipole, one can see visually from Figs 8 and 9 that the binned spectra show systematic residuals, particularly for the $545 \times 857$ and $857 \times 857$ spectra (which are the spectra most contaminated by Galactic dust). Table 4 lists $\chi^{2}$ for each of the binned spectra and best-fitting models shown in Figs 8 and 9. For the power-law CIB template, the $545 \times 857$ and $857 \times 857$ spectra have $\chi^{2}$ values that are $4 \sigma$ and $8 \sigma$ high, respectively. The fits are even worse on mask 50 , where the dust power is roughly double that for mask 40 . We have a partial understanding of the source of these anomalous $\chi^{2}$ values (see Table 7 below). A significant contribution to the high $\chi^{2}$ values comes from our modelling of the Galactic dust contributions to the covariance matrices. As discussed in Section 4, by assuming that the dust is a statistically isotropic Gaussian random field, we are overcounting the effective number of modes and therefore underestimating the dust contribution to the covariance matrices. We defer further discussion of this issue to Section 6.1 where we develop a heuristic model for the sampling variance of Galactic dust that improves the $\chi^{2}$ of the high-frequency spectra significantly, while having very little impact on the model parameters reported in Table 3.

We now consider the effect of replacing the power-law template for the clustered CIB power with the halo model template described in Section 3.1.2. Results are presented in Fig. 9 and in Tables 3 and 4 for mask 40 . The P13 halo model gives a consistently poorer fit to the data than the power-law model on both the full likelihood $\left(\Delta \chi^{2}=135\right)$ and on the binned spectra. The two CIB models are compared in Fig. 10 for the $545 \times 545$ and $857 \times 857$ spectra; this shows clearly the steepening of the halo model template both on large and small scales, which is disfavoured by the data.

The model-fitting approach adopted in this paper relies on the accuracy of the dust and CIB templates. Most of the statistical power of the Planck data lies in the multipole range $\ell \sim 1000-2000$ and so the likelihood will find solutions that minimize the residuals in this multipole range. Using a template of the wrong shape will affect the residuals at both low and high multipoles. At face value, this is what is happening in Fig. 10. Both CIB templates produce reasonably good fits in the multipole range $\ell=1000-2000$, but the halo model template leaves systematic residuals at both high and low multipoles. This suggests that the P13 halo model template is a poor fit to the clustered CIB component at high frequencies. We will discuss this important point in further detail in Section 5.4 after comparing our dust-corrected spectra with those of P13 and with the Herschel/SPIRE measurements of Viero et al. (2013, hereafter V13), the latter extending up to multipoles $\ell \approx 30000$. Note also that the power-law template, despite giving better fits to the data, is only a phenomenological model and there is no reason to expect constant power-law behaviour on all scales. The power-law template deviates from the halo model template on large scales $(\ell \lesssim 300)$, where the physical modelling that underlies the halo model template should be rather robust. One should therefore be cautious not to overinterpret the apparently better fit of the powerlaw template on large scales, particularly given the difficulties in dust removal there and the large sample variance. We present a fuller discussion of the behaviour of these models on large scales in Section 6.2.

\subsection{Joint-mask analysis}

The Fisher matrix analysis of Section 4.1 reveals degeneracies between the clustered and Poisson power of the CIB and the dust power. To reduce the degeneracy with the dust power, we have experimented with combining information from different regions of the sky in an attempt to provide a more accurate separation between the statistically isotropic CIB components and the anisotropic 

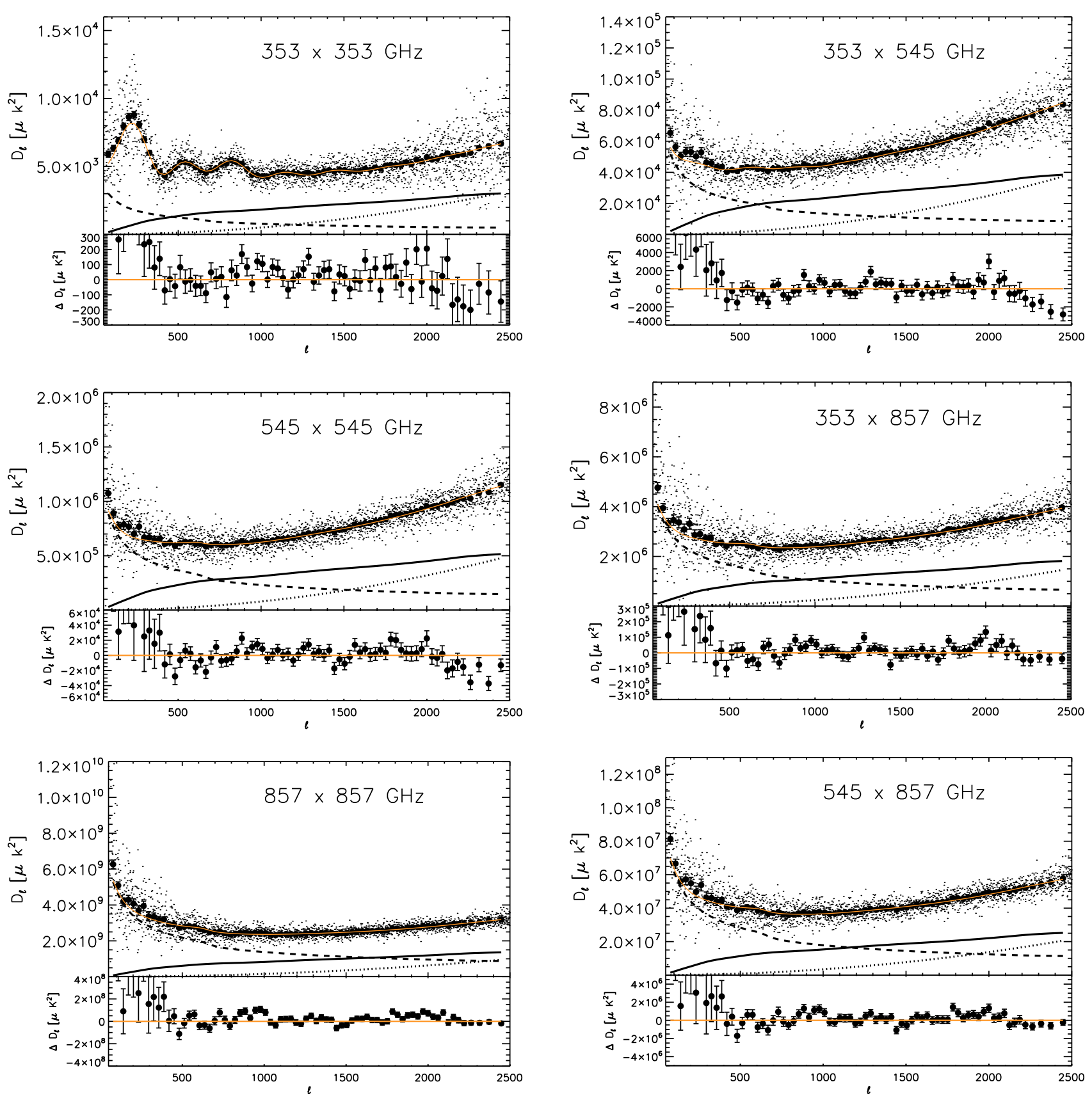

Figure 9. Same as Fig. 8, but with the clustered CIB signal modelled with the P13-based halo model template.

Galactic dust emission. We have therefore performed a 'joint-mask' likelihood analysis using spectra computed on two different masks. We adopt one set of CIB and point source amplitudes for both masks (since these describe statistically isotropic components) but different dust component parameters for each mask (since the dust emission is anisotropic). To do this, we simply include in the data vector the 12 spectra measured on the two masks and compute the appropriate covariance matrix to form a likelihood.

The results of using mask 40 and mask 50 in the joint-mask analysis are given in the sixth column in Table 3 . We can see that the parameters from the joint-mask analysis are very similar, with slightly reduced errors, to those determined individually on mask 40 and mask 50, showing that our results are stable. The main effect is to increase the dust amplitudes determined from mask 40 at 353 and $545 \mathrm{GHz}$ by about $1 \sigma$ (see Fig. 13 below). We found similarly stable parameters using mask 30 and mask 40 in a joint-mask analysis.

\subsection{Comparison with previous measurements}

In Figs 11 and 12, we compare CIB auto and cross-frequency power spectrum measurements at 353,545 and $857 \mathrm{GHz}$ with the most recent measurements from Herschel/SPIRE (V13) and the results from the Planck Collaboration based on the 2013 data (P13). For the data points of $\mathrm{P} 13$, we multiply them by $1.021^{2}, 0.98^{2}$ and $0.96^{2}$ for 353,545 and $857 \mathrm{GHz}$, respectively, to account for the recalibration 
Table 4. Goodness-of-fit tests for the individual auto and cross-frequency spectra, binned with $\Delta \ell=31$. Results are shown for mask 40 and, in each case, are compared to the best-fitting power law and halo model template for the clustered CIB for the appropriate sky fraction from Table 3. For the joint-mask analysis, the goodness of fit of the spectra for mask 40 are shown, but the model is the best-fitting using both masks. The quantity $\Delta \chi^{2}=\chi^{2}-N_{\text {dof }}$ is the difference from the expected $\chi^{2}$, where the number of degrees of freedom $N_{\text {dof }}=74$ in all cases (the number of multipole bins for each spectrum). The fourth and eighth columns list $\Delta \chi^{2}$ in units of the expected dispersion, $\sqrt{2 N_{\mathrm{dof}}}$, and the fifth and ninth columns list the probability to exceed (PTE) the tabulated value of $\chi^{2}$. The spectrum $C_{\ell}^{\text {clean }}$ is a combination of the $857 \times 857,545 \times 857$ and $545 \times 545$ spectra designed to remove most of the Galactic dust emission (see Section 6.2).

\begin{tabular}{|c|c|c|c|c|c|c|c|c|}
\hline Spectrum & $\chi^{2}$ & $\hat{\chi}^{2}$ & $\begin{array}{l}\Delta \chi^{2} / \sqrt{2 N_{\text {dof }}} \\
\text { Power law }\end{array}$ & PTE(per cent) & $\chi^{2}$ & $\hat{\chi}^{2}$ & $\begin{array}{r}\Delta \chi^{2} / \sqrt{2 N_{\text {dof }}} \\
\text { Power law (joint) }\end{array}$ & PTE(per cent) \\
\hline $353 \times 353$ & 101.12 & 1.37 & 2.23 & $! 2.0$ & 95.02 & 1.28 & 1.73 & $! 5.0$ \\
\hline $545 \times 545$ & 126.95 & 1.72 & 4.35 & $! 0.01$ & 102.25 & 1.38 & 2.32 & !1.7 \\
\hline $857 \times 857$ & 168.49 & 2.28 & 7.76 & $<0.01$ & 168.04 & 2.27 & 7.73 & $<0.01$ \\
\hline $353 \times 545$ & 100.23 & 1.35 & 2.16 & $! 2.3$ & 99.40 & 1.34 & 2.09 & $! 2.6$ \\
\hline $353 \times 857$ & 111.51 & 1.51 & 3.08 & $! 0.3$ & 112.86 & 1.53 & 3.19 & $! 0.2$ \\
\hline $545 \times 857$ & 124.94 & 1.69 & 4.17 & $! 0.02$ & 139.32 & 1.88 & 5.37 & $<0.01$ \\
\hline \multirow[t]{2}{*}{$C_{\ell}^{\text {clean }}$} & 114.47 & 1.55 & 3.33 & $! 0.2$ & 101.73 & 1.37 & 2.28 & $! 1.8$ \\
\hline & \multicolumn{4}{|c|}{ Halo model template } & \multicolumn{4}{|c|}{ Halo model template (joint) } \\
\hline $353 \times 353$ & 119.88 & 1.62 & $3.77^{1}$ & $! 0.06$ & 125.81 & 1.70 & 4.26 & $! 0.02$ \\
\hline $545 \times 545$ & 159.35 & 2.15 & 7.02 & $<0.01$ & 158.18 & 2.14 & 6.92 & $<0.01$ \\
\hline $857 \times 857$ & 233.84 & 3.16 & 13.14 & $<0.01$ & 202.66 & 2.74 & 10.58 & $<0.01$ \\
\hline $353 \times 545$ & 157.99 & 2.14 & 6.90 & $<0.01$ & 159.88 & 2.16 & 7.06 & $<0.01$ \\
\hline $353 \times 857$ & 135.73 & 1.83 & 5.07 & $! 0.3$ & 130.38 & 1.76 & 4.63 & $<0.01$ \\
\hline $545 \times 857$ & 164.05 & 2.22 & 7.40 & $<0.01$ & 157.40 & 2.13 & 6.86 & $<0.01$ \\
\hline$C_{\ell}^{\text {clean }}$ & 389.60 & 5.26 & 25.94 & $<0.01$ & 387.07 & 5.23 & 25.73 & $<0.01$ \\
\hline
\end{tabular}
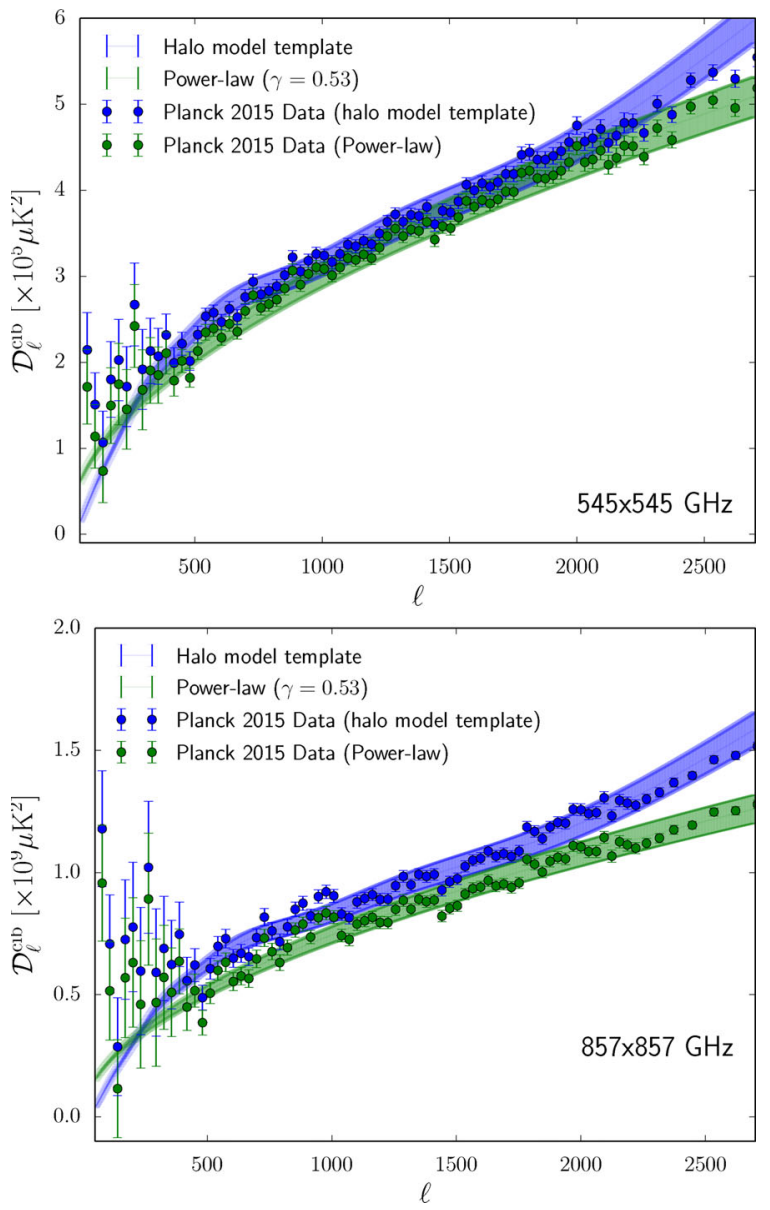

Figure 10. Clustered CIB power spectrum at 545 (top) and $857 \mathrm{GHz}$ (bottom). The data points are the measured spectra for mask 40 after subtracting the best-fitting Poisson and Galactic dust power in the appropriate CIB model. The power-law model, with best-fit slope $\gamma^{\text {cib }}=0.53$, is shown in green and the halo model template in blue. The thickness of the lines correspond to the marginalized $1 \sigma$ error on $A^{\text {cib }}$. between the 2013 and 2015 Planck data. We use the Herschel/SPIRE power spectra with only extended sources masked so that the Poisson contribution is comparable in all three analyses. In addition, we also plot our best-fitting CIB model (assuming the power-law template for the clustered component) and the best-fitting halo model from P13. We plot the total CIB power spectrum measurements, i.e. clustered plus Poisson, and also an estimate of the clustered power. For the latter, we subtract the reported best-fitting Poisson power levels for each measurement. Following P13, we multiply the power spectra of Herschel/SPIRE (data and best-fitting Poisson power levels) at 350 and $500 \mu \mathrm{m}$ by 1.016 and 0.805 , respectively, to colour-correct for comparison with measurements with Planck at 857 and $545 \mathrm{GHz}$. We further multiply the Herschel/SPIRE spectra by calibration factors that account for the SPIRE/Planck relative photometric calibration: $1 / 1.047^{2}$ and $1 / 1.003^{2}$ at 545 and $857 \mathrm{GHz}$, respectively (Bertincourt et al. 2016).

An important result shown in Figs 11 and 12 is the remarkable stability of our results computed on masks 30, 40 and 50. Over this range of masks, the dust power varies by a factor of around 4 , yet the recovered CIB spectra are almost identical. This shows that our likelihood technique can recover the CIB over larger areas of sky and provides incontrovertible evidence for the existence of an isotropic CIB component.

We find generally good agreement among the various analyses for the combined clustering+Poisson signal (shown in the left-hand panels in Figs 11 and 12) and also for the inferred clustered CIB component (shown in the right-hand panels). The latter comparison is more model dependent, requiring fidelity of the respective likelihoods and clustered CIB templates. It is also affected by priors imposed on the Poisson amplitudes. Here the agreement between P13 and our results is less good, particularly for the $353 \times 353$ and $353 \times 545$ spectra. The red lines in Figs 11 and 12 show our best fits for the power-law CIB template. These provide good fits to our spectra. The black lines show the best-fitting halo model as presented in P13. For the combined clustering+Poisson signal, the power-law and halo model fits are nearly identical, but they look quite different for the clustered CIB component. This poses an interesting (and physically important) problem: P13 claim that their 


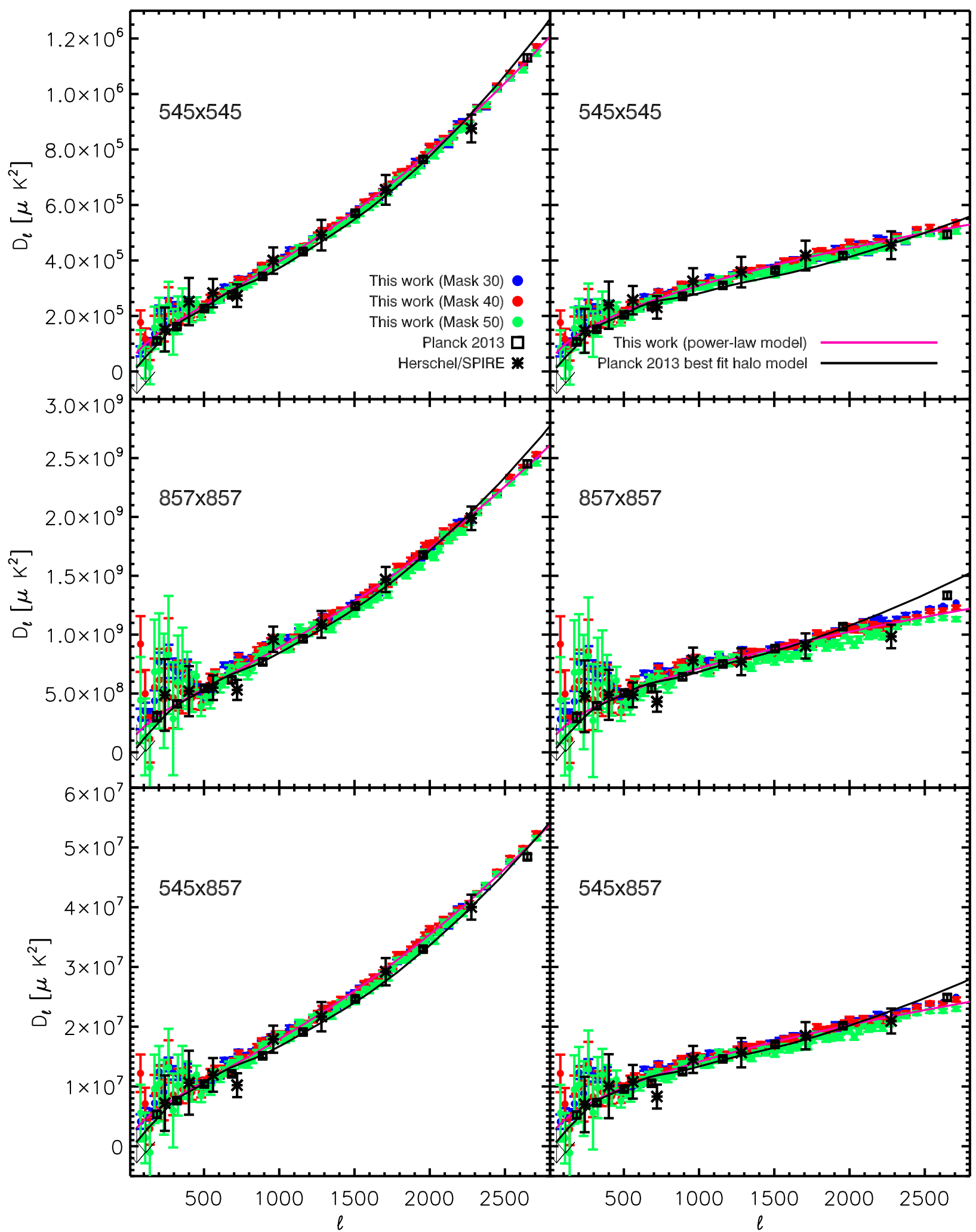

Figure 11. Comparison of our CIB power spectra for $545 \times 545,857 \times 857$ and $545 \times 857$ (top to bottom, respectively) for masks 30 (blue), 40 (red) and 50 (green) with those from Herschel/SPIRE data (black asterisks; V13) and from the 2013 Planck data (black squares; P13). The Herschel/SPIRE measurements have been colour-corrected and recalibrated to match the Planck 545 and $857 \mathrm{GHz}$ bands, as described in the text. The measurements on the left are the total CIB (clustered plus Poisson) power. Also plotted are our best-fitting CIB models for mask 40 assuming the power-law clustering template (red lines) and the best-fitting halo model template from P13 (black lines). The measurements on the right are estimates of the clustered CIB power, obtained by subtracting the reported best-fitting Poisson power levels. 


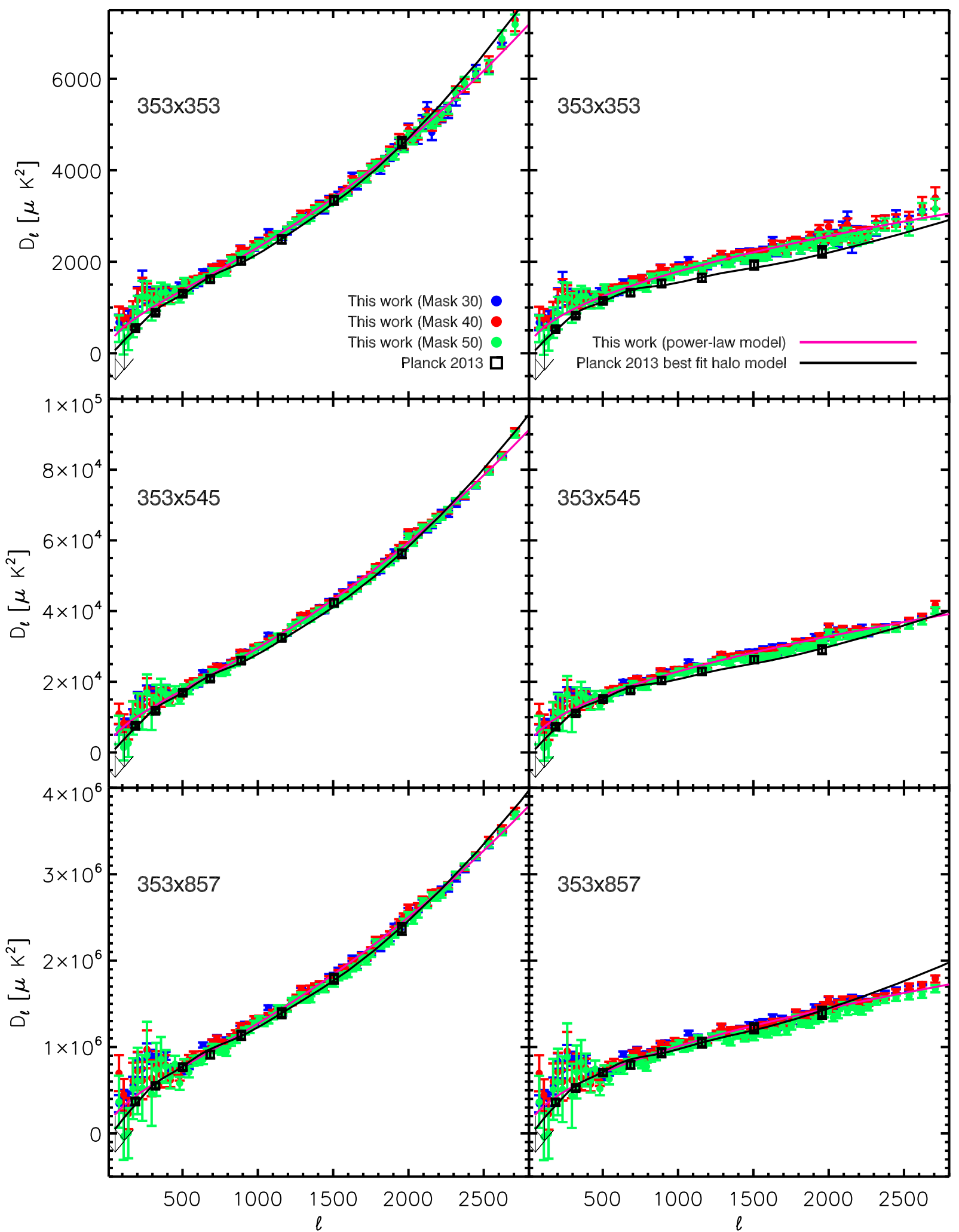

Figure 12. Same as Fig. 11, but for the $353 \times 353,353 \times 545$ and $353 \times 857$ spectra (top to bottom, respectively). Comparisons are done only between this work and P13. 

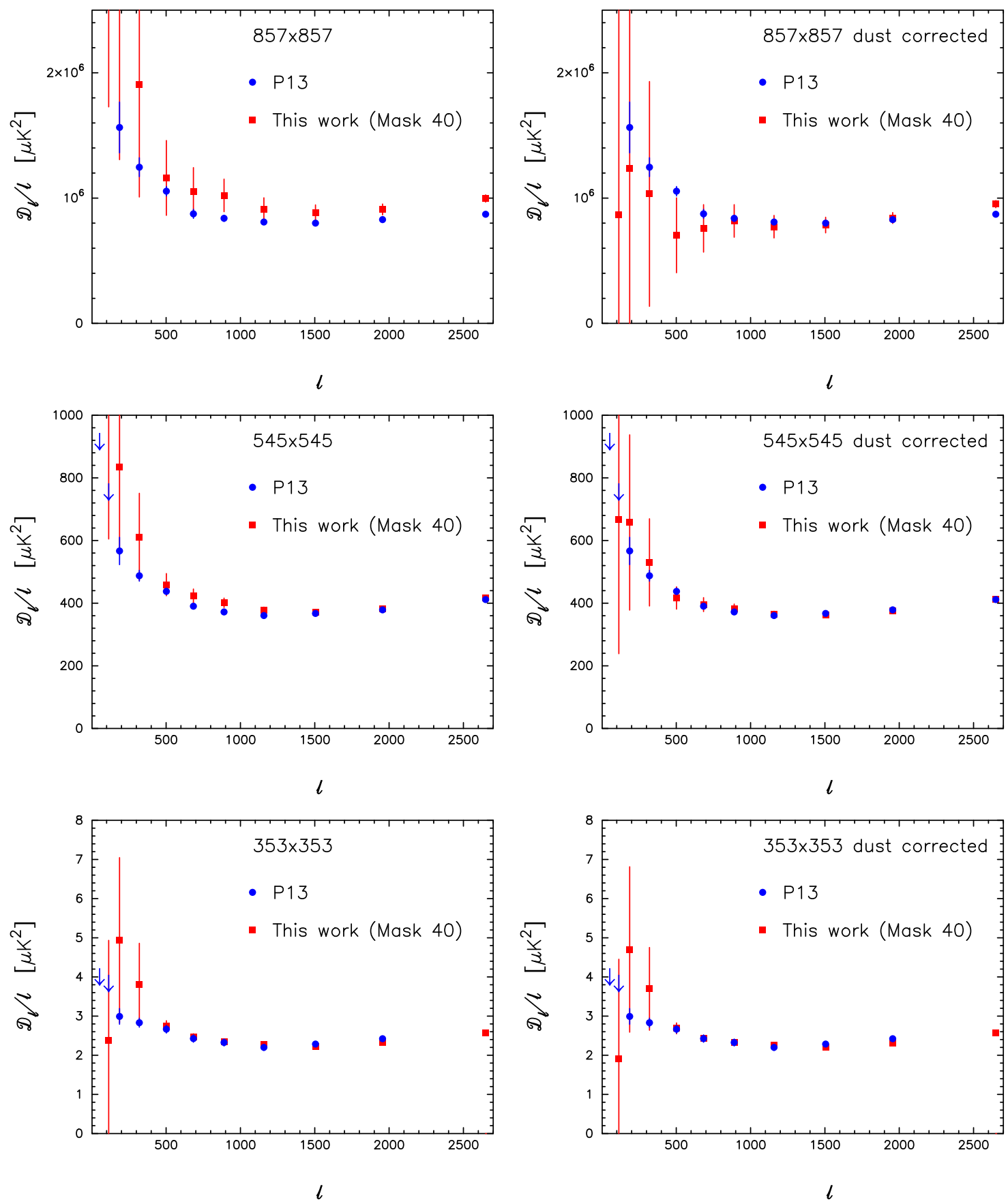

Figure 13. Left: our dust-cleaned spectra (red points) for mask 40, from Fig. 11, but binned in exactly the same way as the P13 spectra shown by the blue points. Right: our dust-cleaned spectra after making small adjustments to the amplitudes of the dust templates chosen to minimize differences with the P13 spectra.

halo model provides a good fit to their spectra, whereas we find that the power-law CIB template provides a consistently better fit to our spectra (as shown in Figs 8-10). Before tackling this apparent inconsistency, we first present a more detailed comparison of our spectra with those of P13. To reduce the numbers of figures, we will present results for the $353 \times 353,545 \times 545$ and $857 \times 857$ spectra only. ${ }^{11}$

\footnotetext{
${ }^{11}$ The behaviour of the cross-frequency spectra can be inferred straightforwardly from the comparisons of the autofrequency spectra.
}

The panels to the left in Fig. 13 show our dust-cleaned spectra (mask 40) compared to the spectra from P13 (from their table D1, with corrections for the change in calibrations from the 2013 to 2015 maps) binned using exactly the same binning as in P13. For our spectra, the dominant source of error in these broad bandpowers is the subtraction of the dust template, which is highly correlated across multipoles. The panels to the right show what happens if the dust amplitudes are allowed to vary, minimizing differences between our spectra and those of P13. For all of the spectra, small shifts upwards in the dust amplitudes $(0.6 \sigma$ for $353 \times 353,1.2 \sigma$ 

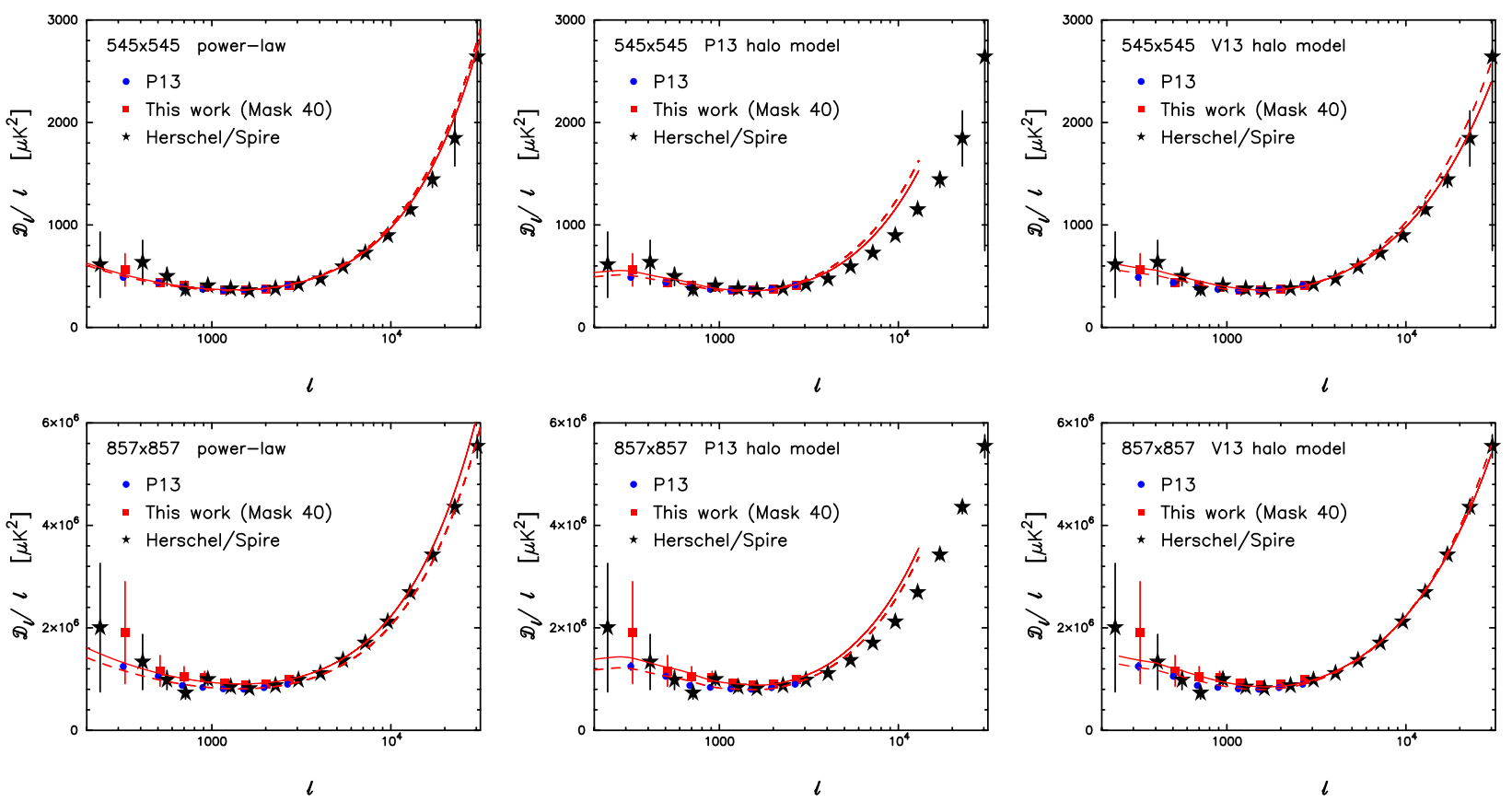

Figure 14. Our spectra (red squares) as plotted in the left-hand panels of Fig. 13 (i.e. with no additional correction to the dust amplitudes) compared to the P13 spectra (blue points) and the Herschel/SPIRE spectra from V13 (black asterisks). The lines show best fits of a Poisson amplitude and clustered CIB template amplitude to each spectrum. The panels to the left show fits assuming a power law-clustered CIB template. The central panels show fits to the P13 halo model template. Panels to the right show fits to a halo model template from V13 (as described in the text). The solid lines in the left and centre panels show fits to our spectra, while the dashed lines show fits to the P13 spectra. These yield nearly identical solutions. The solid lines in the right-hand panels show fits of the V13 template to our spectra combined with the V13 spectra, while the dashed lines show fits to the P13 spectra combined with the V13 spectra.

for $545 \times 545$ and $1.5 \sigma$ for $857 \times 857$ ) bring the spectra into good agreement. It is important to point out that there is some sensitivity of the dust solutions to the shape assumed for the clustered CIB template. Fig. 13 show our results for the power-law CIB template and so it is possible that the small systematic underestimation of the dust amplitudes compared to P13 may be a consequence of a mismatch between the power-law template and the true clustered CIB spectrum. However, any shifts in the dust template amplitudes are small and well within our error budget. We conclude, therefore, that our dust-cleaned spectra, computed over large areas of the sky, are in good agreement with those of P13.

In fact, the small differences in the spectra shown in Fig. 13 have little impact on the physical interpretation of the clustered CIB component. This is illustrated in Fig. 14 that compares the $545 \times 545$ and $857 \times 857$ spectra shown in the left-hand panels of Fig. 13 (i.e. with no further dust correction to our spectra) with the Herschel/SPIRE spectra from V13 plotted up to multipoles $\ell \sim 30000$. The panels to the left show simple $\chi^{2}$ fits of the power law-clustered CIB template plus a point source amplitude to each of our spectra (solid lines) and to the P13 spectra (dashed lines). (We note that for our spectra, these $\chi^{2}$ fits to the binned dustcleaned spectra give CIB and Poisson amplitudes in very good agreement with those obtained from the full likelihood analysis.) The fits to both sets of spectra are nearly identical and are in good agreement with the Herschel/SPIRE spectra at high multipoles. The central panels show what happens if we switch from the powerlaw CIB template to the P13 halo model template. ${ }^{12}$ Again, the fits to both sets of spectra are almost identical and overshoot the

\footnotetext{
12 The P13 halo model templates are tabulated up to a maximum multipole
} of $\ell=13000$.
Herschel/SPIRE spectra by a wide margin. In fact, the $\chi^{2}$ values for the P13 spectra strongly favour the power-law CIB template over the P13 halo model. For nine data points, the power-law CIB model fits to the P13 spectra give $\chi^{2}=3.9$ and 6.4 , respectively, for the $545 \times 545$ and $857 \times 857$ spectra, whereas the P13 halo model fits give $\chi^{2}=33$ and 20 for these spectra. Although the best-fitting halo model in P13 gives an acceptable fit to the full set of spectra over the frequency range $3000-217 \mathrm{GHz}\left(\chi^{2}=100.7\right.$ for 98 data points), it does not give good fits to the $545 \times 545$ and $857 \times 857$ spectra.

The panels to the right in Fig. 14 show $\chi^{2}$ fits to the Planck and Herschel/SPIRE spectra using a $350 \mu \mathrm{m} \times 350 \mu \mathrm{m}$ halo model template from V13. ${ }^{13}$ This template provides good fits to the Planck and Herschel/SPIRE spectra over the full range of multipoles $\ell$ $\approx 200-30000$. Unsurprisingly, over most of the multipole range probed by Planck, the V13 template is closely approximated by a power law with index $\gamma^{\text {cib }}=0.53$. The main difference between the P13 and V13 CIB templates is in the relative amplitudes of the one- and two-halo contributions. The two-halo contribution in the V13 template has a lower amplitude relative to the one-halo term, leading to better fits to the data.

Table 5 compares the Poisson and clustered CIB correlation coefficients determined from our analysis with those measured by P13 and V13. Both components are highly correlated between 545 and $857 \mathrm{GHz}$, as predicted by the Béthermin et al. (2013) models; see column (4) of Table 5. Both our analysis and P13 show a decorrelation of the $353 \mathrm{GHz} \mathrm{CIB}$ and Poisson amplitudes from $857 \mathrm{GHz}$,

\footnotetext{
${ }^{13}$ Specifically, we use the halo model template 1ss14_halo_model_ 350x350_flux_cut_300mJy.txt, available from http://www.astro.caltech. edu/ viero/viero_homepage/toolbox.html.
} 
Table 5. CIB and Poisson power correlation coefficients across frequencies: (1) as measured in this work $(50 \leq \ell \leq 2500)$; (2) P13 (150 $\leq \ell \leq 1000)$, by averaging $C_{\ell}^{v \times v^{\prime}} /\left(C_{\ell}^{v \times v} \times C_{\ell}^{v^{\prime} \times v^{\prime}}\right)^{1 / 2}$; and (3) V13 (100 $\left.\leq \ell \leq 10000\right)$. The measurements are also compared with model predictions (4) at $\ell=1000$ from Béthermin et al. (2013).

\begin{tabular}{lcccc}
\hline$r_{v \times v^{\prime}}^{\text {cib }}$ & $(1)$ & $(2)$ & $(3)$ & $(4)$ \\
\hline $353 \times 545$ & $0.975 \pm 0.005$ & $0.983 \pm 0.007$ & - & 0.99 \\
$353 \times 857$ & $0.892 \pm 0.009$ & $0.911 \pm 0.003$ & - & 0.93 \\
$545 \times 857$ & $0.949 \pm 0.003$ & $0.949 \pm 0.005$ & $0.95 \pm 0.03$ & 0.97 \\
$r_{v \times v^{\prime}}^{\mathrm{ps}}$ & $(1)$ & $(2)$ & $(3)$ & $(4)$ \\
$353 \times 545$ & $0.980 \pm 0.008$ & $0.941 \pm 0.034$ & - & 0.96 \\
$353 \times 857$ & $0.860 \pm 0.011$ & $0.803 \pm 0.054$ & - & 0.83 \\
$545 \times 857$ & $0.970 \pm 0.004$ & $0.897 \pm 0.052$ & $0.98 \pm 0.03$ & 0.92 \\
\hline
\end{tabular}

again in qualitative agreement with the Béthermin et al. (2013) models.

\subsection{Consistency of Poisson amplitudes with source counts}

Table 6 compares our Poisson point source amplitudes (for the power-law CIB model fits) with the values computed in Section 3.4 from measurements of source counts. At 857 and $545 \mathrm{GHz}$, our likelihood analysis favours Poisson amplitudes somewhat lower than the source count predictions. We note that at these frequencies, the predictions are dominated by the counts from Béthermin et al. (2012a). At $353 \mathrm{GHz}$, our best-fitting Poisson power level is about 25 per cent larger than source count prediction based on fits to the counts from Geach et al. (2017), and more than a factor of 2 higher than expected from the APEX/LABOCA and ALMA counts plotted in Fig. 6. However, the source counts of Geach et al. (2017) show no obvious turnover at faint flux densities and so our predicted Poisson power levels are strongly dependent on the extrapolation of the source counts to faint levels.

Table 6 also lists the Poisson point source amplitudes determined by P13 from their halo model fits. The difference in the clustered CIB template will introduce systematic differences in the recovered Poisson amplitudes. In addition, P13 introduced flat priors on the point source amplitudes of \pm 20 per cent around the B12 predictions (listed in the third column of Table 6). In fact, the P13 MCMC fits hit the upper end of their priors at $353 \mathrm{GHz}$ and $545 \mathrm{GHz}$. Despite these differences, qualitatively, the P13 spectra give similar results, namely a larger Poisson amplitude at $353 \mathrm{GHz}$ and somewhat lower Poisson amplitudes at 545 and $857 \mathrm{GHz}$ compared to the number count predictions.

In fact, since the Herschel/Planck spectra extend up to $\ell \approx 30000$, one can derive strict upper limits to the Poisson point source amplitudes. We fitted the three highest multipole bins $(\ell=17064$ $30369)$ of the $350 \times 350$ and $500 \times 500 \mu \mathrm{m}$ 'extended sources masked' spectra given in table 10 of V13 to a constant. This gives the limits listed in the final column of Table 6. The actual Poisson amplitudes could be substantially lower depending on the relative contribution of the one-halo CIB term to the measured spectra. ${ }^{14}$

\footnotetext{
${ }^{14}$ For example, the fits of the V13 template shown in Fig. 14 give Poisson amplitudes of $1130 \pm 35$ and $4410 \pm 185 \mathrm{Jy}^{2} \mathrm{sr}^{-1}$ for the $545 \times 545$ and $857 \times 857$ spectra, respectively. These are substantially lower than the mask 40 numbers listed in Table 6 exacerbating the discrepancy with the number count predictions. This may indicate that the one halo term in V13 template is too steep at high multipoles.
}

The upper limits on the Poisson amplitudes derived from the Herschel/SPIRE spectra are consistent with those determined from the Planck spectra, further suggesting that the number count predictions at 545 and (particularly) $857 \mathrm{GHz}$ overestimate the Poisson amplitudes.

\section{ADDRESSING DEFICIENCIES IN THE LIKELIHOOD ANALYSIS}

The previous section demonstrated that our likelihood analysis produces stable solutions for the CIB power spectra over large areas of the sky, which are in good agreement with the spectra measured in P13. However, there are two notable problems with our approach. The first concerns the high $\chi^{2}$ values reported in Table 4 . The $\chi^{2}$ values increase with frequency, strongly suggesting that our modelling of the contribution of Galactic dust to the covariance matrices is deficient. Section 6.1 develops a simple model for the sample variance of this statistically anisotropic component. The second problem concerns the large sample variance at low multipoles inherent in subtracting dust at the power-spectrum, rather than the map level. This can be seen clearly in Fig. 13. If one wants to recover the CIB spectrum accurately at multipoles $\ell \lesssim 500$, there really is no alternative other than to subtract dust at the map level using a tracer of Galactic emission. As an alternative to $\mathrm{H}$ i cleaning, Section 6.2 explores whether we can exploit the small differences between the Galactic dust and CIB SEDs in the Planck maps to subtract dust whilst retaining information on the CIB.

\subsection{Sample variance of statistically anisotropic Galactic dust}

As discussed in Section 4, we have modelled the sample variance from Galactic dust as if it were a statistically isotropic Gaussian field, with power spectrum appropriate to the given mask. In reality, the statistical anisotropy in the dust emission will increase the sample variance of the dust power spectrum and also the covariance between different multipoles. We noted in Section 5.2 that this may explain the poor $\chi^{2}$ values that we find for the $545 \times 857$ and $857 \times 857$ spectra and the oscillatory residuals at $\ell \lesssim 1000$ seen in Figs 8 and 9. In this section, we present some tests of this hypothesis and develop a simple heuristic model that allows us to account for statistically anisotropic dust in the power spectrum covariance matrix.

We showed in Section 3.3 that in regions of low dust emission, the dust power spectrum measured from mask-difference spectra is very similar in shape. However, the amplitude of the power spectrum varies strongly across the sky. This motivates a simple model for the dust emission as a statistically isotropic, Gaussian process modulated by a large-scale field, i.e. we assume that the dust emission $d(\hat{\boldsymbol{n}})$ varies as

$d(\hat{\boldsymbol{n}})=m(\hat{\boldsymbol{n}})[1+g(\hat{\boldsymbol{n}})]$,

where $g(\hat{\boldsymbol{n}})$ is a statistically isotropic, zero-mean, Gaussian random field and $m(\hat{\boldsymbol{n}})$ is a more slowly varying modulation field. Note that this model has the local variance of dust emission proportional to the square of the local mean emission, consistent with the findings in Miville-Deschênes et al. (2007).

We estimate the modulation field by computing the local variance from smoothed, yearly maps at $857 \mathrm{GHz}$ within circular regions of radius $6^{\circ}$ centred on HEALPIX $N_{\text {side }}=256$ pixels. The covariance matrix for this modulated-Gaussian model can be calculated following the standard calculation for a masked, statistically isotropic Gaussian field (e.g. Efstathiou 2004) but with the mask $w(\hat{\boldsymbol{n}})$ replaced by 
Table 6. Mean values of the Poisson power from the full likelihood analysis and the 68 per cent confidence limits. The prediction from the B12 model, the P13 measurements (see tables 6 and 9 of P13, respectively) and the best-fitting Poisson power from this work (Table 3) are compared here. The final column gives upper limits on the Poisson power levels computed from the Herschel/SPIRE spectra of V13, as described in the text. The numbers are in units of $\mathrm{Jy}^{2} \mathrm{sr}^{-1}$ and, where appropriate, have been colour-corrected to a CIB SED.

\begin{tabular}{lccccc}
\hline Frequency $(\mathrm{GHz})$ & Number count prediction & B12 prediction & P13 measurement & Mask 40 result & V13 limits \\
\hline 353 & $181 \pm 15$ & $225 \pm 45$ & $262 \pm 8$ & $226 \pm 8$ & - \\
545 & $1729 \pm 82$ & $1454 \pm 291$ & $1690 \pm 45$ & $1539 \pm 90$ & $<1775 \pm 63$ \\
857 & $7355 \pm 221$ & $5628 \pm 1126$ & $5364 \pm 343$ & $5929 \pm 428$ & $<6240 \pm 80$ \\
\hline
\end{tabular}

$w(\hat{\boldsymbol{n}}) m(\hat{\boldsymbol{n}})$. In the limit of wide bandpowers, the effective number of modes that determines the power spectrum variance is

$v_{\ell}=(2 \ell+1) \frac{\left\langle w^{2} m^{2}\right\rangle_{\Omega}^{2}}{\left\langle w^{4} m^{4}\right\rangle_{\Omega}}$

and is lower than the variance computed assuming no modulation, which is given by equation 10 with $m(\hat{\boldsymbol{n}})=1$ (Hivon et al. 2002; see also Challinor \& Chon 2005). Here, the angle brackets, $\langle\cdot\rangle_{\Omega}$, denote averages over the sky. The reduction in the number of modes increases the power spectrum variance and the covariance between different multipoles. We defer a fuller discussion of the modulated-Gaussian model and the details behind the derivation of equation (10) and its extension for unbinned spectra, to Appendix C.

We constructed a new likelihood using the modulated-Gaussian model of equation (9) and used this to derive new parameter constraints. The marginalized posterior distributions for the power-law CIB model are shown in Fig. 15 for masks 40 and 50. For most parameters, the changes are small with relative shifts in the peaks of the posteriors much less than $1 \sigma$. As expected, the changes are larger for mask 50 than mask 40 . In fact, at $857 \mathrm{GHz}$ where accurate dust modelling is most critical, the dust correction to the covariance moves the parameters for mask 50 closer to those found for mask 40. There are also substantial improvements in the $\chi^{2}$ of the individual auto- and cross-frequency spectra with the new likelihood, as shown in Table 7 . These results provide further evidence that it is our modelling of dust sampling variance that is mainly responsible for the high $\chi^{2}$ values. Our heuristic model goes a long way in accounting for the high $\chi^{2}$ values, but does not completely eliminate the problem for the $857 \times 857$ spectra.

\subsection{Dust cleaning}

Ideally, one would like to remove the dust emission at the map level to eliminate the dust sample variance entirely. $\mathrm{P} 13$ used $\mathrm{HI}$ as a tracer of dust emission and this appears to work well in clean regions of the sky. At present, the P13 analysis provides the most accurate estimates of the dust cleaning over the multipole range $\ell=145-590 .^{15}$

\footnotetext{
${ }^{15}$ We note that because of the limited angular resolution of the Hi maps (16 arcmin) for the GASS field (P13 also used other $\mathrm{H}_{\text {I }}$ fields having higher angular resolution of $10 \mathrm{arcmin}$, but the GASS field is dominating the analysis), the dust subtraction in P13 is performed at the map level up to $\ell=590$. At larger multipoles, dust is corrected at the power spectrum level using a power-law model for the dust spectrum with an amplitude fitted to the dust spectrum inferred from the H $\mathrm{H}$ maps over the multipole range $\ell=120-590$. Mis-subtraction of dust at the map level will always lead to excess power, which is why P13 quote upper limits to the CIB power spectrum at $\ell<145$. Mis-subtraction of dust at the power spectrum level at higher multipoles can introduce systematic errors of either sign.
}

As an alternative, we have experimented with using a linear combination of the 545 and $857 \mathrm{GHz}$ maps to null dust emission. Consider the 'dust-nulled' map

$M^{\text {cleaned }}=M_{545}-\alpha M_{857}$,

where $\alpha=\sqrt{A_{545}^{\text {dust }} / A_{857}^{\text {dust }}}$ is the ratio of the dust power spectrum amplitudes at these frequencies determined as the best fit of the full likelihood analysis. Here, $M_{545}$ is a $545 \mathrm{GHz}$ map and similarly for $M_{857}$. If the dust were fully coherent between 545 and $857 \mathrm{GHz}$, $M^{\text {cleaned }}$ should contain no dust signal, only CIB, and the power spectrum of the cleaned map should contain no sample variance from dust. Note that this removes from the spectrum both the sample variance from the dust itself and from chance correlations between dust and the CIB. We generate two such maps corresponding to the Year-1 and Year-2 data. Since the maps have different beams, the subtraction is actually done in the power spectrum domain by forming

$$
\begin{aligned}
\hat{C}_{\ell}^{\text {cleaned }}= & \hat{C}_{\ell}^{545, \mathrm{Y} 1 \times 545, \mathrm{Y} 2}-\alpha \hat{C}_{\ell}^{857, \mathrm{Y} 1 \times 545, \mathrm{Y} 2} \\
& -\alpha \hat{C}_{\ell}^{545, \mathrm{Y} 1 \times 857, \mathrm{Y} 2}+\alpha^{2} \hat{C}_{\ell}^{857, \mathrm{Y} 1 \times 857, \mathrm{Y} 2} .
\end{aligned}
$$

The covariance of the $\hat{C}_{\ell}^{\text {cleaned }}$ can be constructed from linear combinations of the elements of the 545 and $857 \mathrm{GHz}$ blocks of the full covariance matrix. By construction, if dust is included in the full covariance matrix with the same scaling factor $\alpha$, the covariance of the $\hat{C}_{\ell}^{\text {cleaned }}$ will contain no dust.

We perform this dust cleaning on three different masks with the cleaning coefficients $\alpha$ summarized in Table 8 . Note that there are small differences in the values of $\alpha$ when fitting the power-law and halo model templates for the clustered CIB in the likelihood; we report both sets of values in Table 8. Fig. 16 shows the dustnulled spectra computed from equation (12) on mask 40 for the power-law and halo model templates. We also show the best-fitting models determined from the respective likelihood analyses, which contain only clustered CIB and Poisson power by construction and the residuals of the measured dust-nulled spectra from these. The $\chi^{2}$ of the dust-nulled spectra with respect to the models are reported in Table 8. Comparing to the raw $545 \times 545$ spectrum in Fig. 8, we see that the cleaning reduces the total power by a factor of around 50 on the largest scales (this is a reduction in CIB power as well as dust power) and the variance of the spectra is significantly reduced. We note that the differences $\delta \alpha$ in cleaning coefficients for the different clustered CIB templates imply only very small differences in the level of dust power that remains in the dut-nulled spectrum: a fraction $(\delta \alpha / \alpha)^{2}$ of the dust power in the $545 \times 545$ spectrum, i.e. around $50 \mu \mathrm{K}^{2}$ on the largest scales compared to around $10^{4} \mu \mathrm{K}^{2}$ from the CIB. Fig. 16 shows that the power-law model provides a better fit to the dust-nulled spectra than the P13 halo model. At high multipoles, the best-fitting P13 halo model sits high compared to the data, in agreement with the results shown in Fig. 10. 

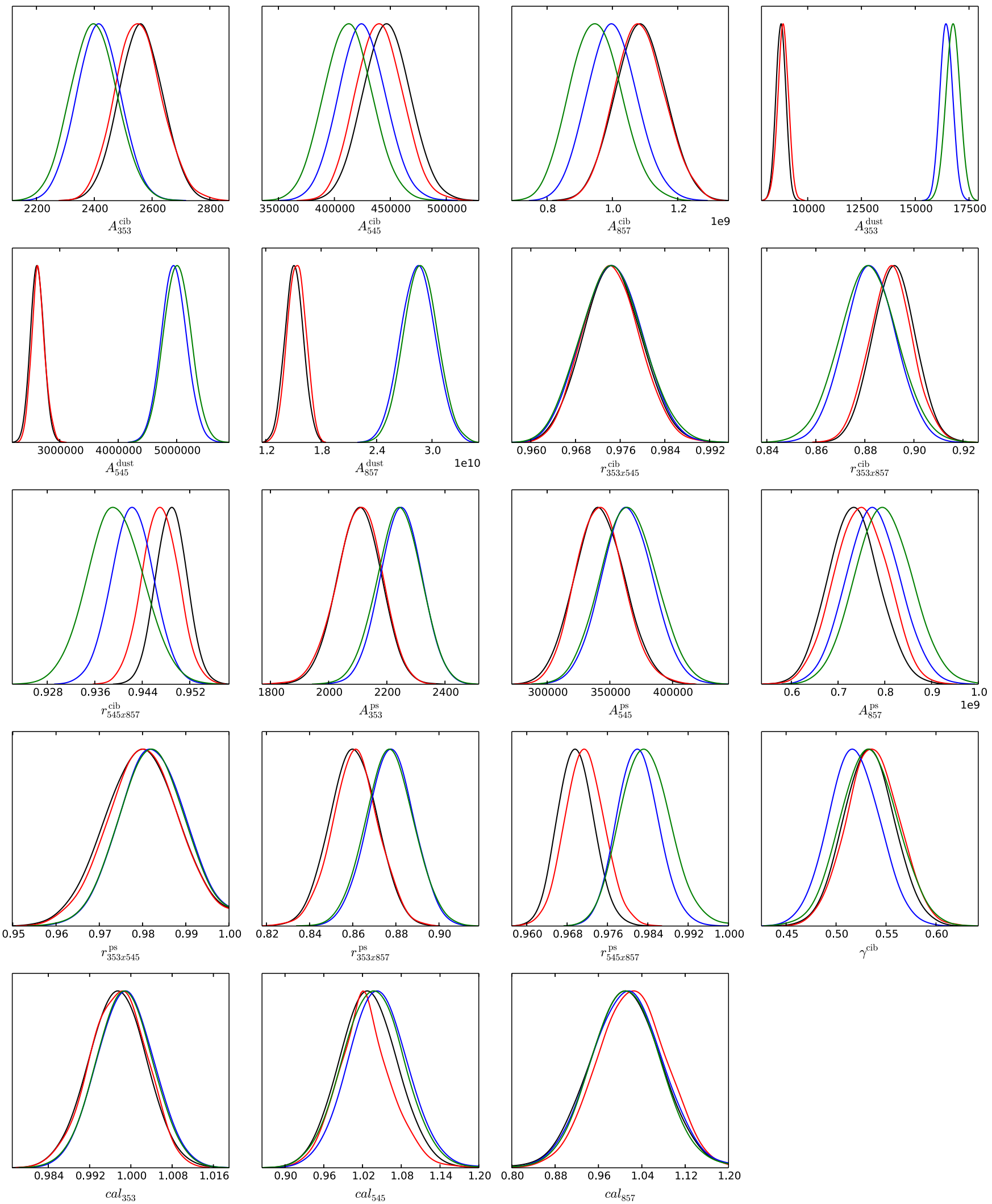

Figure 15. Posterior distributions of the model parameters (for the power law-clustered CIB model) for masks 40 (black) and 50 (blue) and the same after accounting for the statistical anisotropy of Galactic dust in the power spectrum covariance matrix (red for mask 40 and green for mask 50 ). Note that the results from mask 40 are very similar before and after the correction to the covariance matrix. 
Table 7. Goodness-of-fit tests for the individual auto and cross-frequency spectra, binned with $\Delta \ell=31$, with respect to the best-fitting model (power law-clustered CIB) from the full likelihood analysis for masks 40 and 50. The quantities tabulated are the same as in Table 4. Results are shown for the standard covariance matrix ('original'), which reproduce values from Table 4 for convenience and with the correction for statistically anisotropic Galactic dust ('dust-corrected'). The number of degree of freedom is 74 in all cases.

\begin{tabular}{|c|c|c|c|c|c|c|c|c|}
\hline Spectrum & $\chi^{2}$ & $\hat{\chi}^{2}$ & $\Delta \chi^{2} / \sqrt{2 N_{\mathrm{dof}}}$ & PTE(per cent) & $\chi^{2}$ & $\hat{\chi}^{2}$ & $\Delta \chi^{2} / \sqrt{2 N_{\mathrm{dof}}}$ & PTE(per cent) \\
\hline & \multicolumn{4}{|c|}{ Mask 40 (original) } & \multicolumn{4}{|c|}{ Mask 40 (dust-corrected) } \\
\hline $353 \times 353$ & 101.12 & 1.37 & 2.23 & 2.0 & 90.59 & 1.22 & 1.36 & 9.2 \\
\hline $545 \times 545$ & 126.95 & 1.72 & 4.35 & 0.01 & 90.13 & 1.22 & 1.33 & 9.8 \\
\hline $857 \times 857$ & 168.49 & 2.28 & 7.76 & $<0.1$ & 131.32 & 1.77 & 4.71 & 0.1 \\
\hline $353 \times 545$ & 100.23 & 1.35 & 2.16 & 2.3 & 91.63 & 1.24 & 1.45 & 8.1 \\
\hline $353 \times 857$ & 111.51 & 1.51 & 3.08 & 0.3 & 96.70 & 1.31 & 1.87 & 3.9 \\
\hline \multirow[t]{2}{*}{$545 \times 857$} & 124.94 & 1.69 & 4.17 & 0.2 & 107.52 & 1.45 & 2.76 & 0.7 \\
\hline & \multicolumn{4}{|c|}{ Mask 50 (original) } & \multicolumn{4}{|c|}{ Mask 50 (dust-corrected) } \\
\hline $353 \times 353$ & 96.44 & 1.30 & 1.84 & 4.1 & 83.06 & 1.22 & $! 0.74$ & 22.0 \\
\hline $545 \times 545$ & 92.16 & 1.25 & 1.49 & 7.5 & 70.16 & 0.95 & -0.32 & 60.5 \\
\hline $857 \times 857$ & 162.86 & 2.20 & 7.39 & $<0.1$ & 107.07 & 1.45 & $! 2.72$ & 0.7 \\
\hline $353 \times 545$ & 89.77 & 1.21 & 1.30 & 10.2 & 70.81 & 0.96 & -0.26 & 58.4 \\
\hline $353 \times 857$ & 105.48 & 1.43 & 2.59 & 1.0 & 80.40 & 1.09 & $! 0.53$ & 28.6 \\
\hline $545 \times 857$ & 134.27 & 1.81 & 4.95 & $<0.1$ & 93.40 & 1.26 & $! 1.59$ & 6.3 \\
\hline
\end{tabular}

Table 8. Dust-cleaning coefficients $\alpha$ and the $\chi^{2}$ of the cleaned spectrum. These are shown for various masks and for the power-law and halo model templates for the clustered CIB power. The $\chi^{2}$ values, and associated probabilities to exceed (PTE), are with respect to the best-fitting model of the respective full likelihood analysis. The last three columns refer to the fits when a model for the residual dust power from SED variations across the sky (i.e. the blue squares shown in Fig. 16) are accounted for. The number of degrees of freedom is 74 in all cases.

\begin{tabular}{lccccccccc}
\hline Mask & $\alpha$ & $\begin{array}{c}\chi^{2} \\
\text { Power-law model }\end{array}$ & \multicolumn{2}{c}{$\begin{array}{c}\text { PTE(per cent) } \\
\text { Halo model template }\end{array}$} & $\alpha$ & \multicolumn{2}{c}{$\begin{array}{c}\chi^{2} \\
\text { Halo model template+dust residuals }\end{array}$} \\
\hline 30 & 0.0134 & 113.1 & 0.7 & 0.0133 & 348.3 & $<0.1$ & 0.0133 & 222.4 & $<0.1$ \\
40 & 0.0130 & 114.5 & 0.2 & 0.0130 & 389.6 & $<0.1$ & 0.0130 & 236.4 & $<0.1$ \\
50 & 0.0129 & 112.3 & 0.3 & 0.0129 & 492.8 & $<0.1$ & 0.0129 & 305.1 & $<0.1$ \\
\hline
\end{tabular}
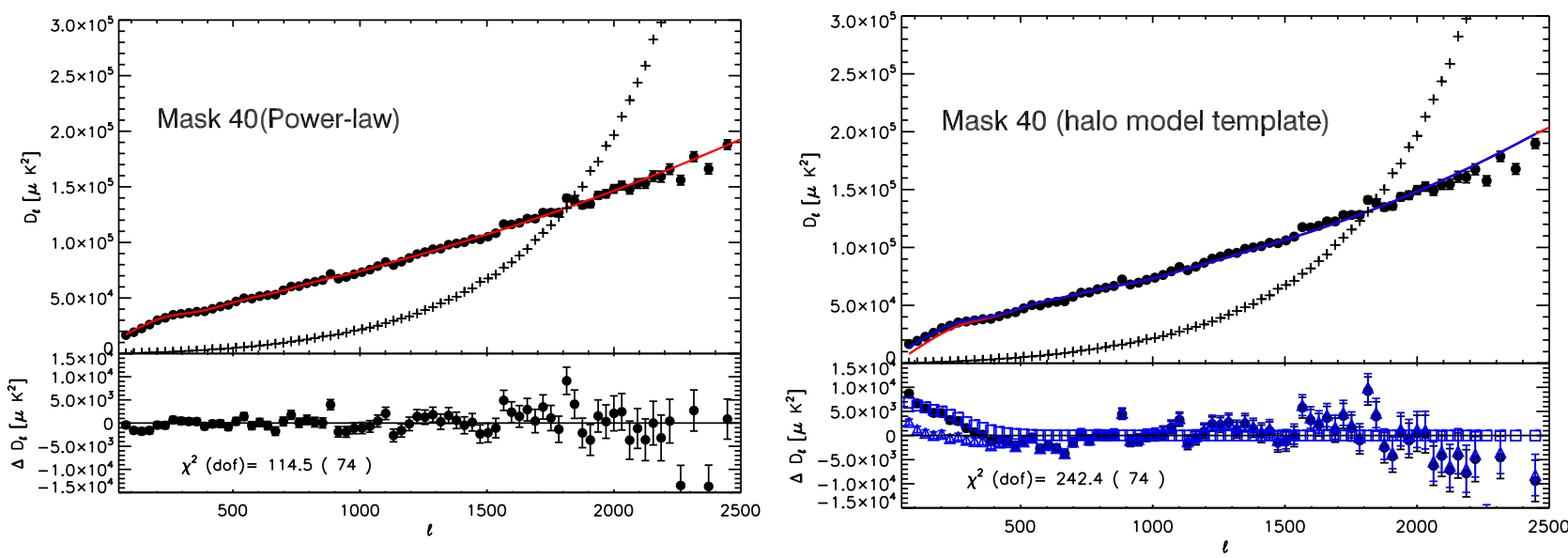

Figure 16. Dust-nulled spectrum for mask 40 computed from equation (12) with values of $\alpha$ taken from Table 8 . We show results for the analysis with a power-law template for the clustered CIB (left) and the halo model template (right). The red lines show the model predictions using the best-fitting parameters of the respective analysis. The data is binned using the same scheme as in Figs 8 and 9. The small black crosses show the noise level of the dust-nulled map of equation (11) computed from the half-ring difference noise maps. The bottom panel of each plot shows the residuals of the data with respect to the model. Error bars are computed from the diagonals of the binned covariance matrices. There are clear excesses at $\ell<300$ in the dust-nulled spectrum with respect to the model prediction for the halo model analysis. These excesses are due to small variations in the SED of the Galactic dust emission over the sky. The blue squares in the bottom panel of the right-hand plot show the power spectrum of the same combination of the 857 and $545 \mathrm{GHz}$ maps measured on the Planck models of dust emission discussed in Section 3.3. This spectrum has been multiplied by a factor of 0.7. With this small rescaling, the power spectrum from the SED variations in the model reproduces the excess observed in the data on large scales. Adding the modelled power from dust-cleaning errors to the CIB model gives the blue line plotted in the top panel. The residuals of the data with respect to this model are shown as the blue triangles in the bottom panel.

Fig. 17 compares our dust-nulled spectrum with the equivalent spectral combination (equation 12) computed from the Herschel/SPIRE measurements of V13. (The errors on the Herschel/SPIRE points are computed by adding the errors of the in- dividual spectra in quadrature as we do not have estimates of the covariances between the component spectra. The errors plotted in Fig. 17 are therefore likely to be overestimated.) The Planck and Herschel/SPIRE spectra match well in the overlap region 1000 


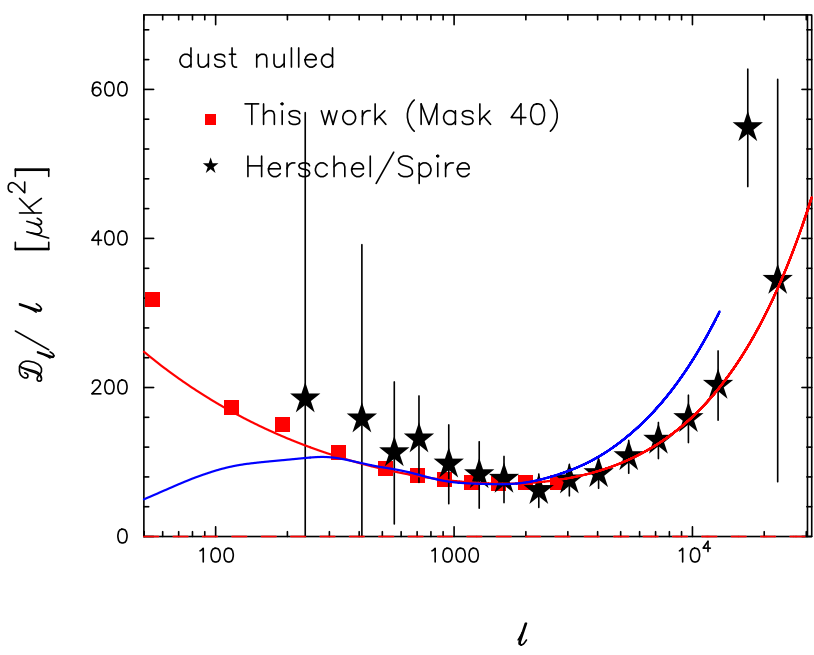

Figure 17. Dust-nulled Planck spectrum for mask 40 (red squares). The black stars show the equivalent dust-nulled spectral combination (equation 12) for the Herschel/SPIRE data from V13. (Note that this refers to the CIB-only spectra in V13.) The red line shows a fit to our data assuming a power law-clustered CIB component. The blue line shows a fit assuming the P13 halo model.

$\lesssim \ell \lesssim 3000$. The solid red line shows the best-fitting power-law model to our spectrum. This fit provides a good match to the Herschel/SPIRE spectrum at high multipoles. The blue line shows the best-fitting P13 halo model. This overshoots the Herschel/SPIRE spectrum at high multipoles. The trends illustrated in Fig. 17 are qualitatively similar to those shown in Fig. 14.

The P13 halo model and power-law model give almost identical fits over the multipole range $\ell=300-1000$. At lower multipoles, the halo model template falls off more steeply. Subtracting dust at the power spectrum level, as in our standard analysis, cannot discriminate between these models at low multipoles because of the high sample variance of Galactic dust (see Fig. 14). The $\mathrm{H}_{\mathrm{I}}$ cleaning applied by $\mathrm{P} 13$ recovers the CIB power spectrum accurately down to multipoles of $\ell \approx 200$, but even at these multipoles, the differences between the two model fits shown in Fig. 17 are quite small. The dust-nulled spectrum plotted in Fig. 17 shows clear excess power at low multipoles that is highly discrepant with the halo model predictions. This raises the question of whether the excess is a property of the clustered CIB or whether it is symptomatic of problems with dust subtraction at large scales. (Recall that errors in dust subtraction at the map level will always produce the excess power.)

In fact, it is very unlikely that the excess is related to the modelling of the CIB. At large scales, probing the two-halo term, the CIB should be proportional to the matter power spectrum with an amplitude that depends on the effective galaxy bias and dust emissivity per unit volume. We experimented fitting the parametric halo model of P13 directly to the cleaned spectrum of Fig. 17, but were unable to find good fits at low multipoles for physically reasonable parameters.

A more plausible explanation of the excess power at low multipoles is the presence of SED variations of the Galactic dust emission over the sky. The cleaned (Year-1) map, $M^{\text {cleaned }}$, is shown in Fig. 18 along with the $545 \mathrm{GHz}$ map. Comparing these maps demonstrates that the frequency combination in $M^{\text {cleaned }}$ does remove dust very effectively away from the Galactic plane. However, even at high Galactic latitude, there are anisotropic features that are clearly not CIB fluctuations. See, for example, the
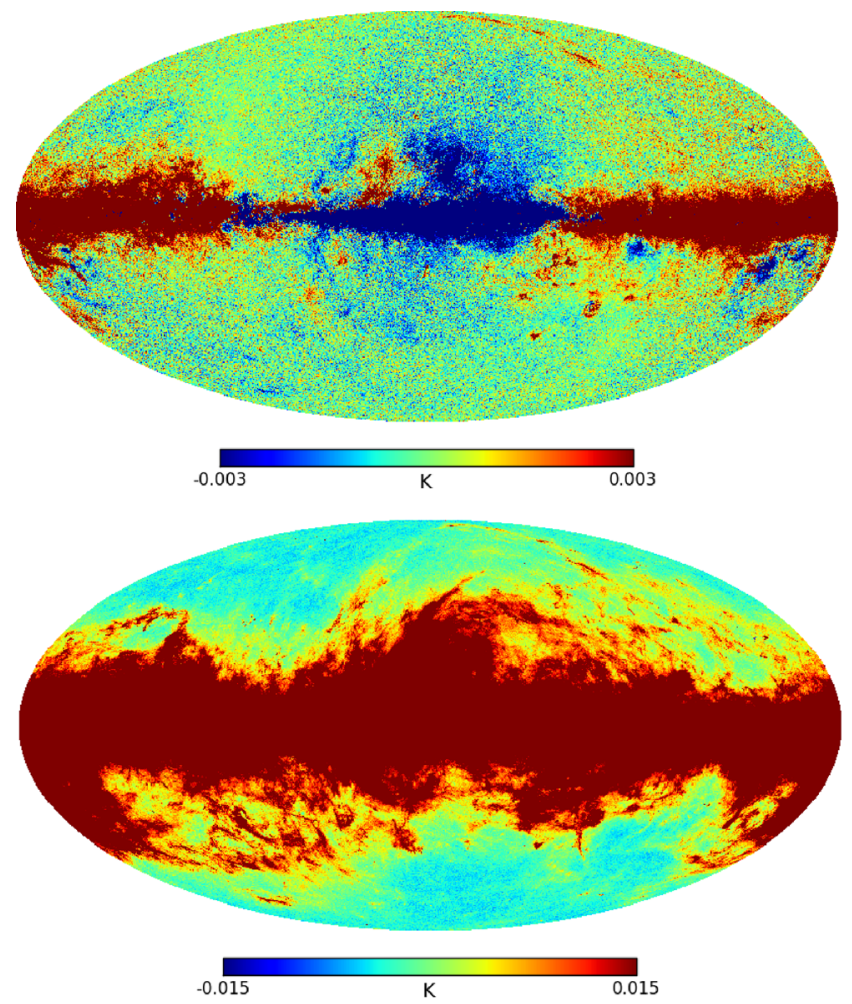

Figure 18. Top: dust-cleaned map, $M_{545}-\alpha M_{857}$. The dust-cleaning coefficient $\alpha=0.0130$ is taken from Table 8 for mask 40 and the power-law CIB template. The colour scale is limited to $[-3 \mathrm{mK},+3 \mathrm{mK}]$ to better reveal the small dust residuals at high Galactic latitude (the residuals in the Galactic plane are saturated). These arise from local variations in the SED of the dust emission. Bottom: the original $545 \mathrm{GHz}$ map, with Galactic mask 40 overlaid, to show how effective the frequency differencing is at cleaning Galactic dust emission at high latitude. Note the different ranges of the colour scale between the two panels. In both cases, the mean measured outside of Galactic mask 40 has been removed.

filamentary structures near the north Galactic pole, which coincide with features in the dust temperature and spectral index maps reconstructed from Planck and IRAS $100 \mu \mathrm{m}$ data in Planck Collaboration XI (2014b).

To demonstrate that small SED variations produce residual power in the cleaned map with the same shape and amplitude that we see in Fig. 16, we form the same frequency difference (i.e. equation 11 with $\alpha=0.0130$ ) of the Planck dust-model maps introduced in Section 3.3 (Planck Collaboration XII 2016c). The SED variations in these maps are derived from the estimates of the dust temperature and spectral index of Planck Collaboration XI (2014b). Since we are interested in large angular scales, we smooth the simulated dust maps as described in Appendix C. The power spectrum of the masked, frequency-differenced dust maps are shown in Fig. 16 (in the lower panel of the right-hand plot). This spectrum has a very similar shape to the excess power over the halo model template that is measured in the data, with a comparable amplitude. In the figure, we have scaled the power spectrum of the modelled dust maps by a factor of 0.7 to bring it closer to the measured excess. If we include this model for residual dust power, the halo model fits well at low multipoles, although the fit at high multipoles is still poor. The purpose of this comparison, however, is not to develop a detailed model of dust-cleaning residuals at low multipoles, but rather to illustrate that dust SED variations of the order determined by Planck Collaboration XI (2014b) (whether one believes them 
or not) can explain the excess power at low multipoles seen in our dust-nulled spectrum.

Figs 16 and 17 strongly suggest that the dust-nulled maps are dominated by CIB emission at multipoles $\ell \gtrsim 300$. A linear combination of the 545 and $857 \mathrm{GHz}$ maps therefore provides a simple way of generating a CIB-dominated map at $\ell \gtrsim 300$ that can be used to cross-correlate with other data sets. Larsen et al. (2016) have used this approach to delens the Planck temperature maps using the CIB as a tracer of the matter distribution. Larsen et al. (2016) actually further reduce the dust contamination in the cleaned maps by masking the filamentary regions near the north Galactic pole visible in Fig. 18.

Finally, we note that we do not expect any significant effect on the results presented in Section 5 from these small variations in the dust SED, since they are dwarfed by the large sampling variance in the dust power spectra at low multipoles (see Fig. 3).

\section{CONCLUSIONS}

The main purpose of this paper has been to adapt the likelihood approach used in the Planck cosmological parameter analysis to recover the power spectrum of CIB anisotropies at frequencies in the range $353-857 \mathrm{GHz}$ over large areas of sky. To achieve this, we have adapted the CAMSPEC power spectrum analysis software to high frequencies. We use mask-difference power spectra, exploiting the anisotropy of Galactic dust emission, to determine a template dust power spectrum that we then fit to measured power spectra together with amplitudes for the clustered and Poisson contributions to the CIB power spectrum (and a number of other 'nuisance' parameters). We have validated our likelihood methodology using simulations and various consistency tests.

We now summarize our main results.

(i) The power spectrum of Galactic dust emission has a nearly universal shape over the frequency range $353-857 \mathrm{GHz}$ over large areas of sky (at least 60 per cent).

(ii) Our likelihood fits recover clustered+Poisson CIB spectra over 30,40 and 50 per cent of sky that are almost identical, even though the dust power varies by a factor of around 4 over this range of sky fractions. This demonstrates the stability of dust subtraction performed at the power spectrum level and also provides incontrovertible evidence for the statistical isotropy of the CIB fluctuations over large areas of sky.

(iii) We fit two models for the clustered CIB: a power-law model with index $\gamma^{\text {cib }}$ and a halo model template based on the best-fitting models of P13. For the power-law model, we find $\gamma^{\text {cib }}=0.53 \pm$ 0.02 , consistent with the values derived as foreground solutions from the Planck CMB likelihood at frequencies $v \leq 217 \mathrm{GHz}$ (Planck Collaboration XV 2014c). Over the frequency range 353-857 GHz, the power-law model is found to be a better fit to our spectra than the P13 halo model.

(iv) Our CIB spectra are consistent with those determined by P13 over around $2000 \mathrm{deg}^{2}$ in regions of low H I column density and therefore low dust emission. Our results are also consistent with the spectra measured by V13 using Herschel/SPIRE data.

(v) Fitting either our spectra or the P13 spectra at 545 and $857 \mathrm{GHz}$ over the multipoles probed by Planck, we find that the P13 halo model overpredicts the Herschel/SPIRE spectra at high multipoles $\ell \gtrsim 3000$. A halo model from V13 gives acceptable fits to the Planck and Herschel/SPIRE data over the entire multipole range $200 \lesssim \ell \lesssim 30000$. The main difference between the P13 and V13 halo models lies in the relative amplitudes of the one- and two-halo terms; the two-halo term in the V13 model has a lower amplitude so that the sum of the two terms is closely approximated by a single power law.

(vi) Our best fits give lower Poisson point source amplitudes at 545 and $857 \mathrm{GHz}$ than those inferred from source counts. Our Poisson amplitudes at these frequencies are consistent with upper limits (but not with the best fits derived by using V13 halo model) derived from the Herschel/SPIRE spectra at multipoles $\ell \sim 30000$. At $353 \mathrm{GHz}$, our best-fitting Poisson point source amplitude is about 25 per cent higher than inferred from recent source counts. However, the source count prediction at this frequency is sensitive to extrapolation of the counts to faint flux densities.

(vii) Our spectra give high $\chi^{2}$ values relative to the best-fitting models. The excess $\chi^{2}$ values correlate strongly with frequency, suggesting that they are caused by our modelling of the dust contribution to the covariance matrices used to construct the likelihood. We have developed a heuristic model to account for the statisticallyanisotropic nature of Galactic dust that substantially reduces, but does not eliminate, the excess $\chi^{2}$ values. The best-fitting dust and CIB parameters hardly change if we use the heuristic model in the likelihood.

(viii) The main drawback of cleaning dust at the power spectrum level is the high sample variance at low multipoles $(\ell \lesssim 500)$. The only way to reduce this sample variance is to subtract dust at the map level. We have experimented with linear combinations of the 545 and $857 \mathrm{GHz}$ maps designed to cancel Galactic dust, exploiting the small differences between the dust and CIB SEDs. The power spectra of such cleaned maps are insensitive to sky fraction and have a shape at multipoles $\ell \gtrsim 500$ consistent with the CIB spectra determined from the 545 and $857 \mathrm{GHz}$ Planck maps with our standard analysis (i.e. subtracting dust at the power spectrum level). This strongly suggests that the dust-nulled linear combination is dominated by CIB emission at multipoles $\ell \gtrsim 300$. The dust-nulled power spectrum from Planck is in excellent agreement with the equivalent combination of spectra measured by Herschel/SPIRE. At $\ell \lesssim 300$, the dust-nulled power spectrum shows excess power over the best-fitting halo model template that is consistent with small dust SED variations over the sky inferred by Planck Collaboration XI (2014b). Recovering the CIB spectrum accurately at multipoles $\ell \lesssim 300$ presents a formidable problem that has yet to be solved. ${ }^{16}$

\section{ACKNOWLEDGEMENTS}

We thank the following for helpful discussions: Marina Migliaccio, Steven Gratton and Diana Harrison on the likelihood analysis; Paolo Serra and Marco Tucci on the CIB modelling and halo-model codes; and Alexander Karim on the sources counts from ALMA and SCUBA. Some of the results in this paper have been derived using the HEALPIX package (Górski et al. 2005). This work is based on observations obtained with Planck (http://www.esa.int/Planck), an ESA science mission with instruments and contributions directly funded by ESA Member States, NASA and Canada. GL acknowledge financial support from 'Programme National de Cosmologie and Galaxies'(PNCG) of CNRS/INSU, France, the OCEVU Labex (ANR-11-LABX-0060) and the *AMIDEX project (ANR-11IDEX-0001-02) funded by the 'Investissements d'Avenir' French

\footnotetext{
${ }^{16}$ We note that $\mathrm{H}$ i cleaning, as applied by $\mathrm{P} 13$ in areas of low $\mathrm{H}$ i column density, leads to upper limits to the CIB power spectrum at multipoles $\ell \lesssim 200$.
} 
government program managed by the ANR. DSY Mak acknowledges hospitality from the Laboratoire d'Astrophysique de Marseille, where part of this work was completed.

\section{REFERENCES}

Amblard A. et al., 2010, A\&A, 518, L9

Bertincourt B. et al., 2016, A\&A, 588, A107

Béthermin M., Dole H., Cousin M., Bavouzet N., 2010, A\&A, 516, A43

Béthermin M., Dole H., Lagache G., Le Borgne D., Penin A., 2011, A\&A, 529, A4

Béthermin M. et al., 2012a, A\&A, 542, A58

Béthermin M. et al., 2012b, ApJ, 757, L23 (B12)

Béthermin M., Wang L., Doré O., Lagache G., Sargent M., Daddi E., Cousin M., Aussel H., 2013, A\&A, 557, A66

Challinor A., Chon G., 2005, MNRAS, 360, 509

Clements D. L. et al., 2010, A\&A, 518, L8

Coppin K. et al., 2006, MNRAS, 372, 1621

Dunkley J. et al., 2011, ApJ, 739, 52

Dunkley J. et al., 2013, J. Cosmol. Astropart. Phys., 7, 25

Efstathiou G., 2004, MNRAS, 349, 603

Foreman-Mackey D., Hogg D. W., Lang D., Goodman J., 2013, PASP, 125, 306

Geach J. E. et al., 2017, MNRAS, 465, 1789

Giannantonio T., Scranton R., Crittenden R. G., Nichol R. C., Boughn S. P., Myers A. D., Richards G. T., 2008, Phys. Rev. D, 77, 123520

Glenn J. et al., 2010, MNRAS, 409, 109

Górski K. M., Hivon E., Banday A. J., Wandelt B. D., Hansen F. K., Reinecke M., Bartelmann M., 2005, ApJ, 622, 759

Grossan B., Smoot G. F., 2007, A\&A, 474, 731

Haiman Z., Knox L., 2000, ApJ, 530, 124

Hajian A. et al., 2012, ApJ, 744, 40

Hall N. R. et al., 2010, ApJ, 718, 632

Hatsukade B., Ohta K., Seko A., Yabe K., Akiyama M., 2013, ApJ, 769, L27

Hivon E., Górski K. M., Netterfield C. B., Crill B. P., Prunet S., Hansen F., 2002, ApJ, 567, 2

Karim A. et al., 2013, MNRAS, 432, 2

Lagache G., Bavouzet N., Fernandez-Conde N., Ponthieu N., Rodet T., Dole H., Miville-Deschênes M.-A., Puget J.-L., 2007, ApJ, 665, L89

Larsen P., Challinor A., Sherwin B. D., Mak D., 2016, Phys. Rev. Lett., 117, 151102

Lawrence A. et al., 2007, MNRAS, 379, 1599

Lewis A., Bridle S., 2002, Phys. Rev. D, 66, 103511

Matsuura S. et al., 2011, ApJ, 737, 2

Miville-Deschênes M.-A., Lagache G., Puget J.-L., 2002, A\&A, 393, 749

Miville-Deschênes M.-A., Lagache G., Boulanger F., Puget J.-L., 2007, A\&A, 469, 595

Miville-Deschênes M.-A. et al., 2010, A\&A, 518, L104

Oliver S. J. et al., 2010, A\&A, 518, L21

Pénin A. et al., 2012, A\&A, 543, A123

Planck Collaboration XVIII, 2011a, A\&A, 536, A18 (PEP)

Planck Collaboration XXIV, 2011b, A\&A, 536, A24

Planck Collaboration VII, 2013, A\&A, 550, A133

Planck Collaboration VIII, 2014a, A\&A, 571, A8

Planck Collaboration XI, 2014b, A\&A, 571, A11

Planck Collaboration XV, 2014c, A\&A, 571, A15

Planck Collaboration XXVIII, 2014d, A\&A, 571, A28

Planck Collaboration XXX, 2014e, A\&A, 571, A30 (P13)

Planck Collaboration VIII, 2016a, A\&A, 594, A8

Planck Collaboration XI, 2016b, A\&A, 594, A11

Planck Collaboration XII, 2016c, A\&A, 594, A12

Planck Collaboration XLVI, 2016d, A\&A, 596, A107

Planck Collaboration XLVIII 2016e, A\&A, 596, A109

Serra P., Lagache G., Doré O., Pullen A., White M., 2014, A\&A, 570, A98

Simpson J. M. et al., 2015, ApJ, 807, 128

Story K. T. et al., 2013, ApJ, 779, 86 van Dokkum P. G. et al., 2006, ApJ, 638, L59

Viero M. P. et al., 2009, ApJ, 707, 1766

Viero M. P. et al., 2013, ApJ, 772, 77 (V13)

Weiß A. et al., 2009, ApJ, 707, 1201

Zehavi I. et al., 2002, ApJ, 571, 172

\section{APPENDIX A: COVARIANCE MATRICES AND LIKELIHOOD VALIDATION}

\section{A1 Mask-coupling and covariance matrices}

The construction of the coupling matrices, $M_{\ell \ell^{\prime}}$, which relate the theory angular power spectra to the mean of the pseudo-spectra and the covariance of the pseudo-spectra, follows, for example, Efstathiou (2004).

Consider the cross-spectrum between two maps, labelled by $i$ and $j$, with independent noise realizations. We allow for different masks, $w^{i}(\hat{\boldsymbol{n}})$ and $w^{j}(\hat{\boldsymbol{n}})$, to be applied to these maps before taking the crossspectrum. The mean of the pseudo-spectrum between these maps is

$\left\langle\tilde{C}_{\ell}^{i j}\right\rangle=\sum_{\ell^{\prime}} M_{\ell \ell^{\prime}}\left[w^{i} \times w^{j}\right] C_{\ell^{\prime}}^{i j}$,

where $C_{\ell}^{i j}$ is the theory cross-spectrum (smoothed by the appropriate beam transfer functions) and

$M_{\ell_{1} \ell_{2}}\left[w^{i} \times w^{j}\right]=\frac{2 \ell_{2}+1}{4 \pi} \sum_{\ell_{3}}\left(2 \ell_{3}+1\right) W_{\ell_{3}}^{i j}\left(\begin{array}{ccc}\ell_{1} & \ell_{2} & \ell_{3} \\ 0 & 0 & 0\end{array}\right)^{2}$

$$
=\left(2 \ell_{2}+1\right) \Xi\left(\ell_{1}, \ell_{2}, W^{i j}\right) .
$$

Here, the cross-spectrum of the masks is

$W_{\ell}^{i j}=\frac{1}{2 \ell+1} \sum_{m} w_{\ell m}^{i}\left(w_{\ell m}^{j}\right)^{*}$,

where $w_{\ell m}^{i}$ are the spherical multipoles of mask $i$, and similarly for $w_{\ell m}^{j}$.

For the covariance matrices of the pseudo-spectra, we assume that the theory power spectra are smooth on the scale over which the cross-spectra of the masks have significant support. This allows us to approximate the covariance matrices as

$$
\begin{aligned}
\operatorname{cov}\left(\tilde{C}_{\ell}^{i j}, \tilde{C}_{\ell^{\prime}}^{p q}\right) \approx & \sqrt{C_{\ell}^{i p} C_{\ell^{\prime}}^{i p}} \sqrt{C_{\ell}^{j q} C_{\ell^{\prime}}^{j q}} \Xi\left(\ell, \ell^{\prime}, W^{(i p)(j q)}\right) \\
& +\sqrt{C_{\ell}^{i p} C_{\ell^{\prime}}^{i p}} \Xi\left(\ell, \ell^{\prime}, W_{\sigma}^{(i p)(j q)}\right) \\
& +\sqrt{C_{\ell}^{j q} C_{\ell^{\prime}}^{j q}} \Xi\left(\ell, \ell^{\prime}, W_{\sigma}^{(j q)(i p)}\right) \\
& +\Xi\left(\ell, \ell^{\prime}, W_{\sigma \sigma}^{(i p)(j q)}\right)+p \leftrightarrow q,
\end{aligned}
$$

where

$$
\begin{aligned}
& W_{\ell}^{(i p)(j q)}=\frac{1}{2 \ell+1} \sum_{m} w_{\ell m}^{(i p)}\left(w_{\ell m}^{(j q)}\right)^{*}, \\
& W_{\sigma, \ell}^{(i p)(j q)}=\frac{1}{2 \ell+1} \sum_{m} w_{\ell m}^{(i p)}\left(w_{\sigma, \ell m}^{(j q)}\right)^{*}, \\
& W_{\sigma \sigma, \ell}^{(i p)(j q)}=\frac{1}{2 \ell+1} \sum_{m} w_{\sigma, \ell m}^{(i p)}\left(w_{\sigma, \ell m}^{(j q)}\right)^{*},
\end{aligned}
$$


and $w_{\ell m}^{(i j)}$ are the spherical multipoles of the product of masks $i$ and $j$ and

$w_{\sigma, \ell m}^{(i j)}=\delta_{i j} \int w^{i}(\hat{\boldsymbol{n}}) w^{j}(\hat{\boldsymbol{n}}) \sigma_{i}^{2}(\hat{\boldsymbol{n}}) \Omega_{\mathrm{pix}} \mathrm{d} \hat{\boldsymbol{n}}$,

with $\sigma_{i}^{2}$ the noise variance in map $i$ in a pixel of area $\Omega_{\text {pix }}$. The noise terms in equation (A5) assume that the noise is Gaussian and uncorrelated between pixels. In practice, we generalize these terms to account approximately for correlated noise following the procedure described in appendix A.8 of Planck Collaboration XV (2014c).

\section{A2 Likelihood validation}

We have validated our likelihood by performing tests on simulated data.

\section{A2.1 Simulations}

We generate simulations of the 353,545 and $857 \mathrm{GHz}$ maps for the appropriate data splits (e.g. yearly or detector-sets) using Gaussian models of the signal and realistic Planck noise. For each of these simulations, we repeat the same spectral and likelihood analysis as for the data. The template for the dust power spectrum and the covariance matrix are fixed to those that we use for the data.

We compute the six theory auto and cross-frequency power spectra using the parameters of the best-fitting power-law CIB model for mask 40 (Section 5). We make simulated maps at 353, 545 and $857 \mathrm{GHz}$ by drawing Gaussian realizations from these spectra that are correctly correlated across frequencies (see, e.g. Giannantonio et al. 2008 and Serra et al. 2014). The maps are then smoothed with the appropriate beams, approximating these as circularly symmetric, given by the autofrequency beam transfer functions. Finally, noise realizations for the required data split from the (8th) Planck full focal plane simulation set (Planck Collaboration XII 2016c) are added to the beam-smoothed maps. Maps are simulated at the same resolution as the data, i.e. HEALPIX $N_{\text {side }}=2048$.

While these simulations can be used to test a number of steps in the construction of the likelihood, including the covariance matrices and the assumption that the measured power spectra are Gaussian distributed, they are not designed to test our assumptions of the dust modelling. In particular, by simulating dust as a statistically isotropic Gaussian field that is fully correlated across frequencies, the simulations conform with the assumptions made in the (baseline) likelihood by construction. It would be interesting in future work
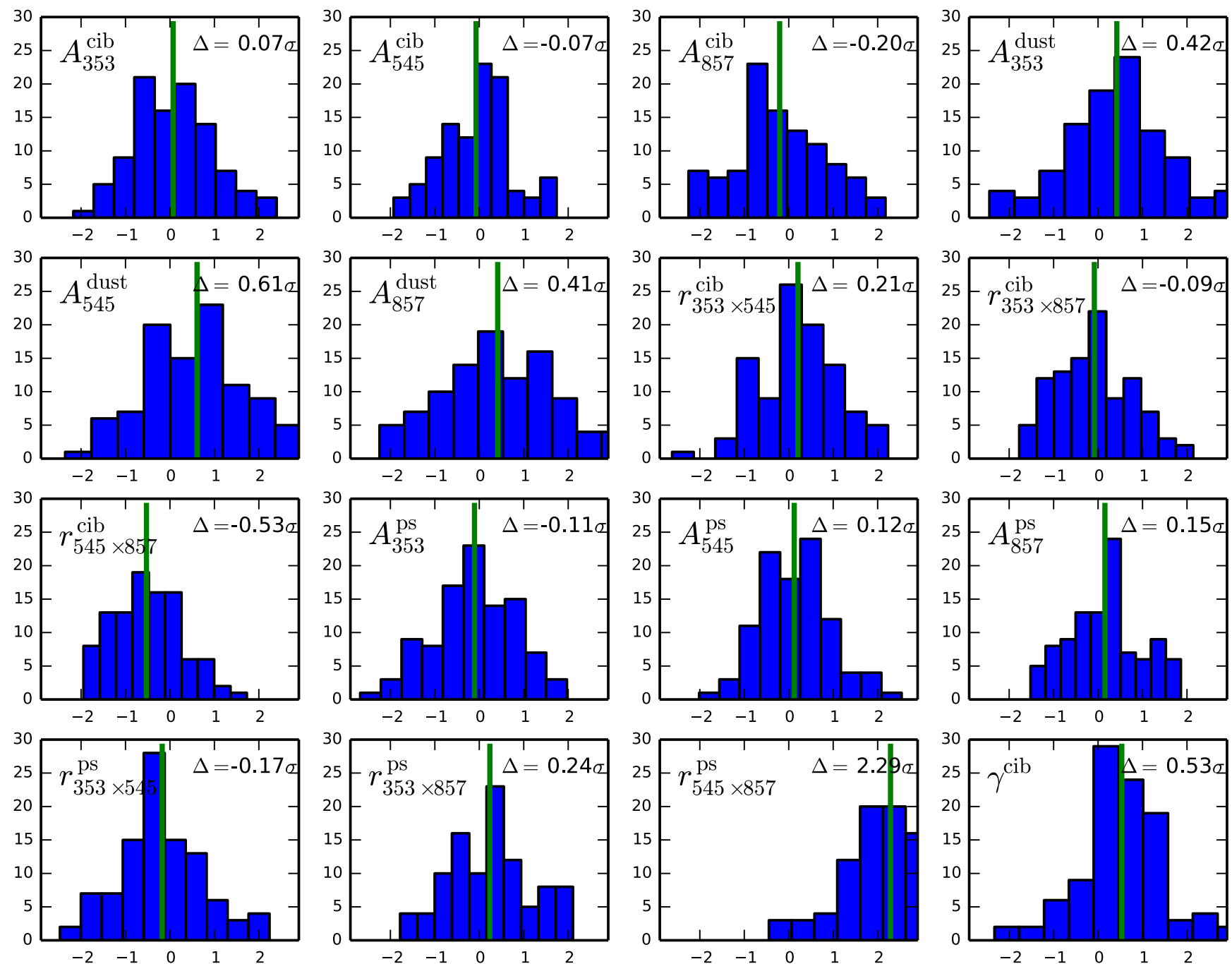

Figure A1. Distributions of the differences between the parameter means and the input values across 100 simulations. The differences are expressed relative to the standard deviations determined from the data (for the same processing as the simulations). The averages of the differences across the simulations are shown by the green lines and correspond to the fractional biases quoted in each panel. 
to use more realistic dust simulations to test these assumptions in detail.

\section{A2.2 Parameter recovery}

For each simulation, we compute all auto and cross-frequency spectra and construct a likelihood from which we sample the parameters of the power-law model using COSMOMC (Lewis \& Bridle 2002). The posterior mean, $p_{\text {sim }}$, is estimated for each simulation, and compared to the input value, $p_{\text {input }}$. We quantify any bias through the quantities $\Delta \equiv\left(p_{\text {sim }}-p_{\text {input }}\right) / \sigma_{\text {data }}$, where $\sigma_{\text {data }}$ is the standard deviation of the posterior distribution for the parameter determined from the data (processed in the same way as the simulations). Ideally, we expect $\Delta$ should scatter across simulations with a mean close to zero and standard deviation around unity. The former tests for bias in the recovered parameters while the latter tests that the posterior means scatter in accordance with the widths of the posterior distributions (i.e. whether the error model in the likelihood is correct).

The resulting distributions of the $\Delta$ across the 100 simulations are shown in Fig. A1, with the average value of $\Delta$ quoted in each panel. The biases are generally small compared to the statistical errors and the scatter of the posterior means across simulations is roughly in line with expectations. An exception is the Poisson correlation coefficient, $r_{545 \times 857}^{\mathrm{ps}}$, which shows a large relative bias of $2.29 \sigma$. However, the absolute bias is only around 1 per cent since the statistical error is very small. For this reason, we have not investigated the source of bias in this parameter in detail.

\section{APPENDIX B: POISSON POWERS FROM SOURCE COUNTS}

\section{B1 Data}

For $353 \mathrm{GHz}$, our main analysis is based on source count measurements from Geach et al. (2017). These are based on the S2CLS, the largest survey to date at a wavelength of $850 \mu \mathrm{m}$, which covers around $5 \mathrm{deg}^{2}$ of extragalactic survey fields (an order of magnitude larger than any previous survey at this wavelength). Geach et al. (2017) present a catalogue of nearly 3000 sub-mm sources, each detected with significance greater than $3.5 \sigma$. Their counts are in reasonable agreement with previous surveys, but are higher than some previous counts by almost a factor of 2 at flux densities $S<10 \mathrm{mJy}$.

To illustrate the differences between earlier source counts at faint flux densities, we also plot counts from Weiß et al. (2009), Simpson et al. (2015) and Karim et al. (2013) in Fig. 6. Briefly, these data are as follows.

(i) Weiß et al. (2009) estimate counts from the LABOCA Extended Chandra Deep Field South (ECDFS) field covering $0.35 \mathrm{deg}^{2}$, using $P(D)$ analysis. ${ }^{17}$ They present a catalogue of 126 sub-mm galaxies detected with a significance level above 3.7 $\sigma$.

(ii) Simpson et al. (2015) analyse 30 ALMA maps centred on bright sub-millimetre sources selected from the United Kingdom Infrared Telescope Infrared Deep Sky Survey Ultra-Deep Survey field (Lawrence et al. 2007) of the S2CLS [the median flux density is $S=(8.7 \pm 0.4) \mathrm{mJy}]$. They find that these SCUBA bright sources are comprised of a blend of multiple sources brighter than $1 \mathrm{mJy}$ in the ALMA maps. The multiplicity of the sources means that the intrinsic number counts originally measured with SCUBA measurements (e.g. Coppin et al. 2006) are likely overestimated and that many of the sources detected by SCUBA are in fact multiple fainter sources.

${ }^{17}$ A statistical method used to estimate the number counts in a field from the pixel histogram of an extragalactic map. (iii) Karim et al. (2013) follow up sources from the LABOCA ECDFS sub-millimeter survey with high-resolution ALMA observations. Their results are in broad agreement with those of the LABOCA survey, but show a deficit at the bright end $(S \gtrsim 2 \mathrm{mJy})$ caused by multiplicity of bright sources. There is evidence from observations at other wavelengths (e.g. $K$-band-selected galaxies at $z>2$; van Dokkum et al. 2006) that the ECDFS is underdense by a factor of around 2 (see Weiß et al. 2009). We therefore follow the suggestion by Weiß et al. (2009) and Karim (private communication) to scale up the source count measurements of Karim et al. (2013) by a factor of 2. In fact, making this correction brings their counts into better agreement with SCUBA and other observations with ALMA (e.g. Hatsukade et al. 2013).

For 545 and $857 \mathrm{GHz}$, we use counts based on Herschel/SPIRE data at 500 and $350 \mu \mathrm{m}$, respectively. We take counts from several works that use different analyses and sources selected from different fields, as follows.

(i) Glenn et al. (2010): number counts are reconstructed based on $P(D)$ analysis using the sources in the Great Observatories Origins Deep Survey North, Lockman-North and Lockman-SWIRE field from the Herschel Multi-tiered Extragalactic Survey (HerMES).

(ii) Béthermin et al. (2012a): field selection is similar to Glenn et al. (2010), but the counts are reconstructed based on stacking analysis.

(iii) Oliver et al. (2010): field selection is similar to Glenn et al. (2010), with additional fields FLS and A2218, but the counts are directly measured from the resolved sources.

(iv) Clements et al. (2010): sources are selected from the largest Herschel survey, ATLAS, which covers $550 \mathrm{deg}^{2}$ and separate fields from the HerMES. The counts are directly measured from resolved sources.

For high flux densities, $S>1 \mathrm{Jy}$, was also use the number counts measured in the Planck high frequency instrument (HFI) bands from Planck Collaboration VII (2013). As noted in Section 3.4, the contribution to the Poisson power from the bright counts are subdominant.

\section{B2 Infrared source count model}

To model the faint source counts, we fit a double power law to the data:

$$
\frac{\mathrm{d} N}{\mathrm{~d} S}=A\left[\left(\frac{S}{B}\right)^{n_{1}}+\left(\frac{S}{B}\right)^{n_{2}}\right]^{-1} .
$$

This model has four free parameters, $A, B, n_{1}$ and $n_{2}$, which we fit to the faint source counts using a Gaussian likelihood. Since $n_{1}$ and $n_{2}$ are degenerate with each other, we apply priors on both parameters: $6 \leq n_{1} \leq 10$ and $0 \leq n_{2} \leq 2.5$, at all three frequencies. We treat each data set as independent and give them equal weight in the likelihood, neglecting any correlations between the differential source counts in different flux-density bins. We apply correction factors of 1.016 and 0.805 at 857 and $545 \mathrm{GHz}$, respectively, to the derived Poisson power in order to convert from Herschel to Planck frequencies. We additionally apply cross-calibration factors of $1 / 1.047^{2}$ and $1 / 1.003^{2}$ at 545 and $857 \mathrm{GHz}$, respectively, that account for the SPIRE/HFI relative gains (Bertincourt et al. 2016).

For the bright flux densities, the differential source count distribution can be described by a single power law:

$$
\frac{\mathrm{d} N}{\mathrm{~d} S}=p S^{-2.5} .
$$


Table B1. Best-fitting parameters of the source count model.

\begin{tabular}{lccccc}
\hline Frequency & $A\left(\mathrm{Jy}^{-1} \mathrm{sr}^{-1}\right)$ & $B(\mathrm{Jy})$ & $n_{1}$ & $n_{2}$ & $p\left(\mathrm{Jy}^{1.5} \mathrm{sr}^{-1}\right)$ \\
\hline 353 & $2.82_{-0.50}^{+0.75} \times 10^{8}$ & $0.007_{-0.004}^{+0.004}$ & $6.5_{-0.5}^{+0.5}$ & $2.42_{-0.13}^{+0.06}$ & $17.24_{-1.63}^{+1.64}$ \\
545 & $7.44_{-0.28}^{+0.44} \times 10^{8}$ & $0.015_{-0.002}^{+0.002}$ & $4.80_{-0.2}^{+0.2}$ & $1.64_{-0.18}^{+0.16}$ & $121.44_{-11.75}^{+11.68}$ \\
857 & $2.88_{-0.56}^{+0.70} \times 10^{8}$ & $0.028_{-0.002}^{+0.002}$ & $5.20_{-0.50}^{+0.30}$ & $1.80_{-0.07}^{+0.07}$ & $565.15_{-45.78}^{+45.72}$ \\
\hline
\end{tabular}

We therefore add this contribution to equation (B1), adding an additional free parameter $p$ (the Euclidean plateau level). We summarize the best-fitting parameters of this model in Table B1 and show in Figs B1-B3, the constraints on the five parameters at each frequency.

\section{APPENDIX C: MODELLING THE POWER SPECTRUM COVARIANCE FOR STATISTICALLY ANISOTROPIC DUST}

\section{C1 The modulated dust model and power spectrum covariance}

In Section 6.1, we introduced a simple model for statistically anisotropic dust emission: $\mathrm{d}(\hat{\boldsymbol{n}})=m(\hat{\boldsymbol{n}})[1+g(\hat{\boldsymbol{n}})]$, where $g(\hat{\boldsymbol{n}})$ is a statistically isotropic, zero-mean Gaussian field with power spectrum $C_{\ell}^{g}$, and $m(\hat{\boldsymbol{n}})$ is a more slowly varying modulation field. We begin by considering the sample variance of power spectrum estimates derived from masked versions of $\mathrm{d}(\hat{\boldsymbol{n}})$.

If the mask $w(\hat{\boldsymbol{n}})$ and the modulation field $m(\hat{\boldsymbol{n}})$ are sufficiently slowly varying, the expected value of the pseudo-spectrum is approximately

$\left\langle\tilde{C}_{\ell}^{d}\right\rangle=\left\langle w^{2} m^{2}\right\rangle_{\Omega} C_{\ell}^{g}$,

where $\langle\cdot\rangle_{\Omega}$ denotes an average over the sky. We remind the reader that the pseudo-spectrum is formed from the spherical multipoles $\tilde{d}_{\ell m}$ of the masked signal as $\tilde{C}_{\ell}^{d}=\sum_{m}\left|\tilde{d}_{\ell m}\right|^{2} /(2 \ell+1)$. The maskdeconvolved spectrum $\hat{C}_{\ell}^{d}$ is approximately a renormalized version of the pseudo-spectrum, $\hat{C}_{\ell}^{d} \approx \tilde{C}_{\ell}^{d} /\left\langle w^{2}\right\rangle_{\Omega}$. In the mean,

$\left\langle\hat{C}_{\ell}^{d}\right\rangle \approx \frac{\left\langle w^{2} m^{2}\right\rangle_{\Omega}}{\left\langle w^{2}\right\rangle_{\Omega}} C_{\ell}^{g}$,

which is mask-dependent because of the modulation field $m(\hat{\boldsymbol{n}})$. It is this quantity that is modelled in the likelihood with the fitting function of equation (5). In the limit of wide bandpowers (such that $\Delta \ell$ is large compared to the support of the power spectrum of the product of the mask and modulation fields), the bandpower variance of the pseudo-spectrum is (Hivon et al. 2002)

$\operatorname{var}\left(\tilde{C}_{\ell}^{d}\right) \approx \frac{2}{(2 \ell+1) \Delta \ell}\left\langle w^{4} m^{4}\right\rangle_{\Omega}\left(C_{\ell}^{g}\right)^{2}$.

Renormalizing to give the variance of the $\hat{C}_{\ell}^{d}$ and expressing the result in terms of $\left\langle\hat{C}_{\ell}^{d}\right\rangle$, we find

$\operatorname{var}\left(\hat{C}_{\ell}^{d}\right) \approx \frac{2}{(2 \ell+1) \Delta \ell} \frac{\left\langle w^{4} m^{4}\right\rangle_{\Omega}}{\left\langle w^{2} m^{2}\right\rangle_{\Omega}^{2}}\left\langle\hat{C}_{\ell}^{d}\right\rangle^{2}$

It follows that the effective number of degrees of freedom is $v_{\ell} \approx$ $(2 \ell+1) \Delta \ell / H[m]$, where

$H[m] \equiv \frac{\left\langle w^{4} m^{4}\right\rangle_{\Omega}}{\left\langle w^{2} m^{2}\right\rangle_{\Omega}^{2}}$ plays the role of an effective inverse sky fraction for the variance. $H[m]$ is larger than its equivalent, $\left\langle w^{4}\right\rangle_{\Omega} /\left\langle w^{2}\right\rangle_{\Omega}^{2}$, in the absence of modulation, reducing $v_{\ell}$ and increasing the variance of the power spectrum.

\section{C2 Approximating the modulation field}

We estimate the modulation field $m(\hat{\boldsymbol{n}})$ using the Planck $857 \mathrm{GHz}$ data on intermediate and large scales as a tracer of dust emission. The modulation controls both the local mean level of dust emission, $\langle d\rangle_{\mathrm{R}}$, and the local variance, $\sigma_{\mathrm{R}}^{2}$. Here, the local mean and variance are calculated within circular apertures of radius $R$. For small $R$ compared to the scale of the modulation, the model predicts $\sigma_{\mathrm{R}}^{2} \propto$ $\langle d\rangle_{\mathrm{R}}^{2}$. This scaling is consistent with the results reported in MivilleDeschênes et al. (2007) in regions of low emission. We estimate the local mean and variance of the $857 \mathrm{GHz}$ maps as follows.

(i) We first smooth the masked, $857 \mathrm{GHz}$ Year-1 and Year-2 maps with a Gaussian of FWHM $=1^{\circ}$ in order to suppress the small-scale CIB and instrument noise.

(ii) Since we are interested in the large-scale modulation field, we estimate this at lower resolution (HEALPIX $N_{\text {side }}=256$ ). At the centre of each $N_{\text {side }}=256$ pixel that lies outside the Galactic mask, we compute the mean of each smoothed map and the cross-variance (to avoid noise bias) within a circular aperture of radius $R=6^{\circ}$. We note that the smoothed maps are retained at their native resolution and pixels in these maps that are masked by the point source mask are discarded.

Fig. C1 shows the variance map and the map of the squared mean calculated with this procedure. The square of the modulation field can be estimated from these maps up to an irrelevant normalization. The variance and mean-squared maps both show significant variation across the sky and are clearly correlated. We note that the mean is sensitive to the estimation of the zero-point level of the maps and the CIB monopole, while the variance is not. In the following, we use the variance map as a proxy for $m^{2}(\hat{\boldsymbol{n}})$.

Evaluating $H[\mathrm{~m}]$ from equation (C5), we find $H=5.77$ for mask 40. This is enhanced by a factor of 1.94 by the modulation, so we expect roughly a factor of $\sqrt{1.94} \approx 1.39$ increase in the bandpower errors on scales where dust is dominant. For a crude estimate of how much the increased errors might reduce the $\chi^{2}$ of the best-fitting model, we simply inflate the bandpower errors of the $857 \times 857$ spectrum by 40 per cent at multipoles $\ell<1000$. Keeping the bestfitting model unchanged, we find that $\hat{\chi}^{2}=1.77$ for 74 degrees of freedom, with a PTE of 0.1 per cent. The equivalent value without modelling the dust modulation (from Table 4) is $\hat{\chi}^{2}=2.28$ so the correction is very significant. Repeating for mask 50 , we find a similar boost of 1.42 in the bandpower errors on large scales. At this mask, we apply the correction to $\ell<1500$ since dust dominates out to higher multipoles and obtain an improved $\hat{\chi}^{2}=1.46$ (again, for 74 degrees of freedom) corresponding to a $\Delta \chi^{2}=56$. A bigger effect is seen for mask 50 since the dust emission is more intense. 


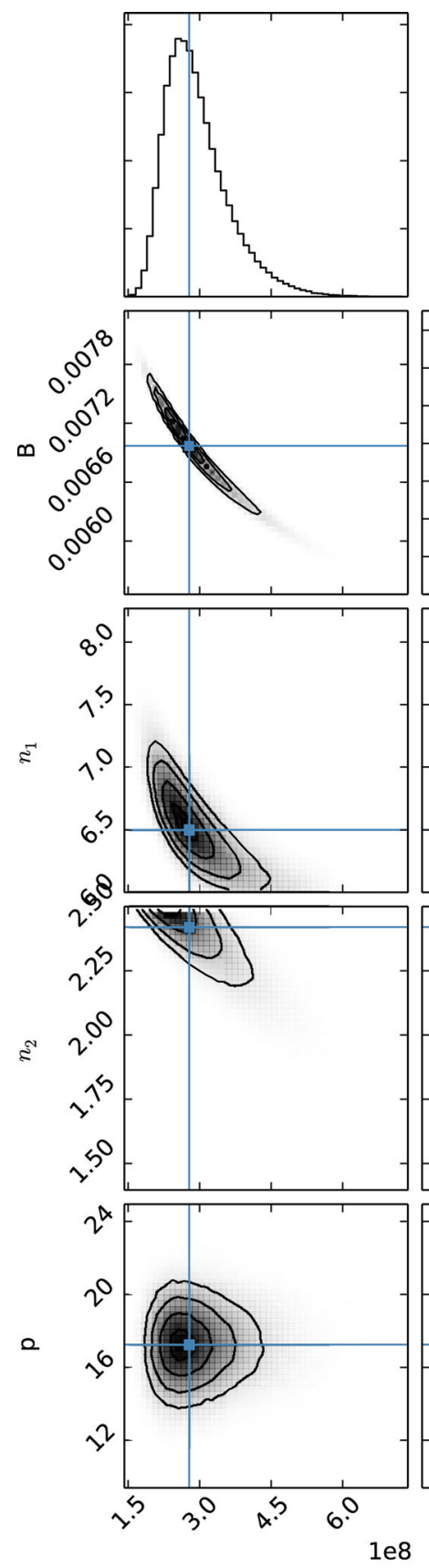

A
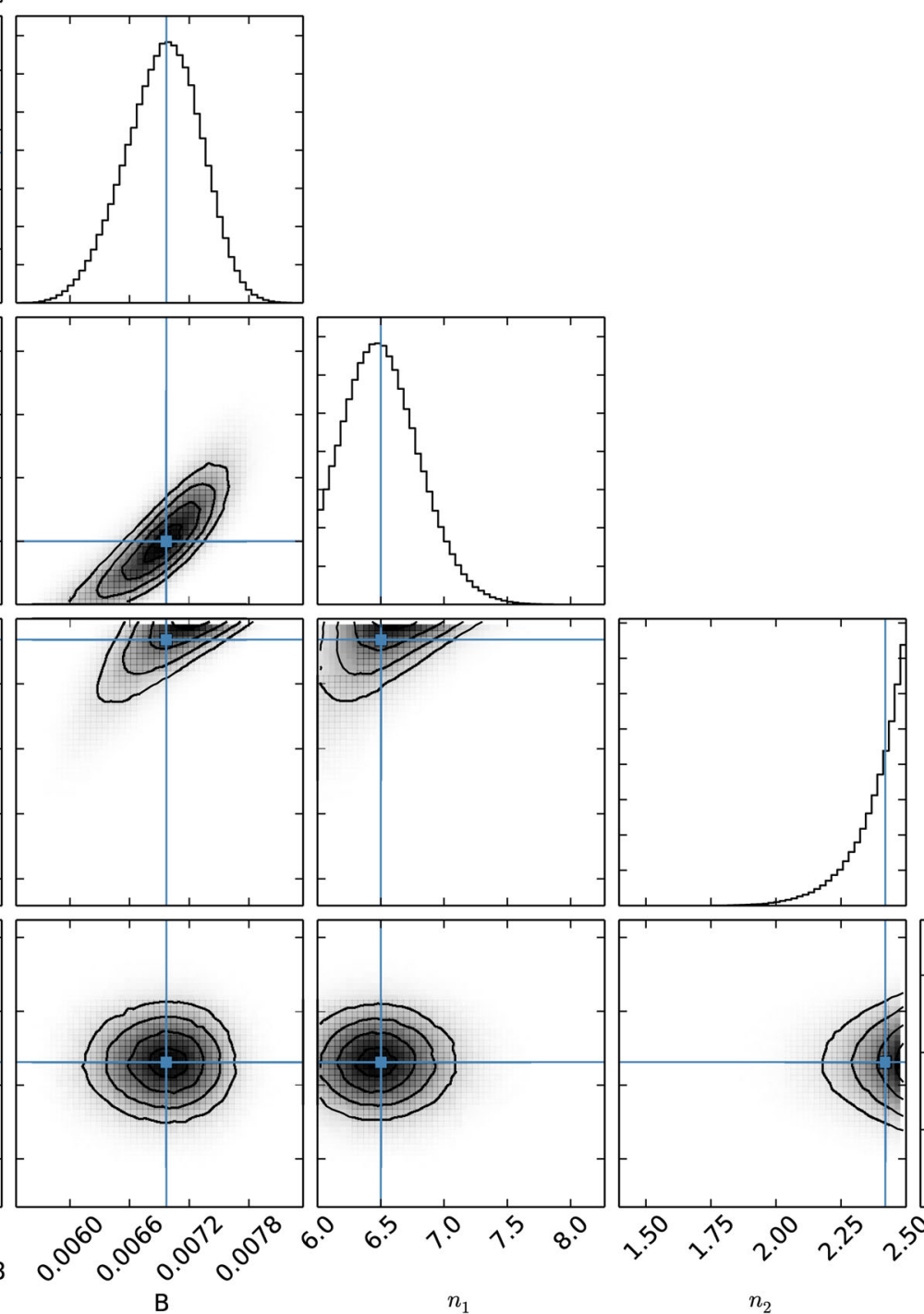

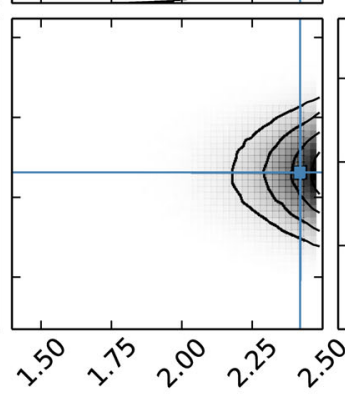

$n_{2}$

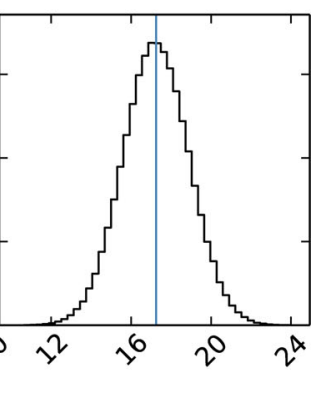

Figure B1. Constraints on the five-parameter source count model at $353 \mathrm{GHz}$. The blue dots indicate the best-fitting values to the MCMC chains.

\section{C3 Corrections to covariance}

Equation (C4) gives the approximate sample variance of the anisotropic dust in broad bandpowers. In the likelihood results presented in Section 6.1, we use a more sophisticated approach whereby we include the dust modulation field directly in the covariance matrices of the pseudo-spectra. We split the signal into the statistically anisotropic dust, $d$, and statistically isotropic components (CIB and CMB) with total theory power spectra $C_{\ell}^{\text {iso, } i j}$ for maps $i$ and $j$. We model the dust as a modulated Gaussian field. For the modulation that is smooth on the beam scale, the convolution with the instrument beam commutes with the modulation, and so the modulation can be treated as an additional mask that is applied to the beam-smoothed, statistically isotropic Gaussian field $g^{i}(\hat{\boldsymbol{n}})$. This modifies the covariance matrix of the pseudo-spectra; for ex- ample, the signal-signal part of the covariance matrix (i.e. the first term on the right of equation A5) becomes

$$
\begin{aligned}
& \operatorname{cov}\left(\tilde{C}_{\ell}^{i j}, \tilde{C}_{\ell^{\prime}}^{p q}\right) \supset \sqrt{C_{\ell}^{\mathrm{iso}, i p} C_{\ell^{\prime}}^{\mathrm{iso}, i p}} \sqrt{C_{\ell}^{\mathrm{iso}, j q} C_{\ell^{\prime}}^{\mathrm{iso}, j q}} \Xi\left(\ell, \ell^{\prime}, W^{(i p)(j q)}\right) \\
& +\sqrt{C_{\ell}^{\text {iso, } i p} C_{\ell^{\prime}}^{\text {iso }, i p}} \sqrt{C_{\ell}^{g, j q} C_{\ell^{\prime}}^{g, j q}} \Xi\left(\ell, \ell^{\prime}, W_{\text {mod. }}^{(i p)(j q)}\right) \\
& +\sqrt{C_{\ell}^{\text {iso, } j q} C_{\ell^{\prime}}^{\text {iso }, j q}} \sqrt{C_{\ell}^{g, i p} C_{\ell^{\prime}}^{g, i p}} \Xi\left(\ell, \ell^{\prime}, W_{\text {mod. }}^{(j q)(i p)}\right) \\
& +\sqrt{C_{\ell}^{g, i p} C_{\ell^{\prime}}^{g, i p}} \sqrt{C_{\ell}^{g, j q} C_{\ell^{\prime}}^{g, j q}} \Xi\left(\ell, \ell^{\prime}, W_{\text {mod. mod. }}^{(j q)(i p)}\right) \\
& +p \leftrightarrow q,
\end{aligned}
$$




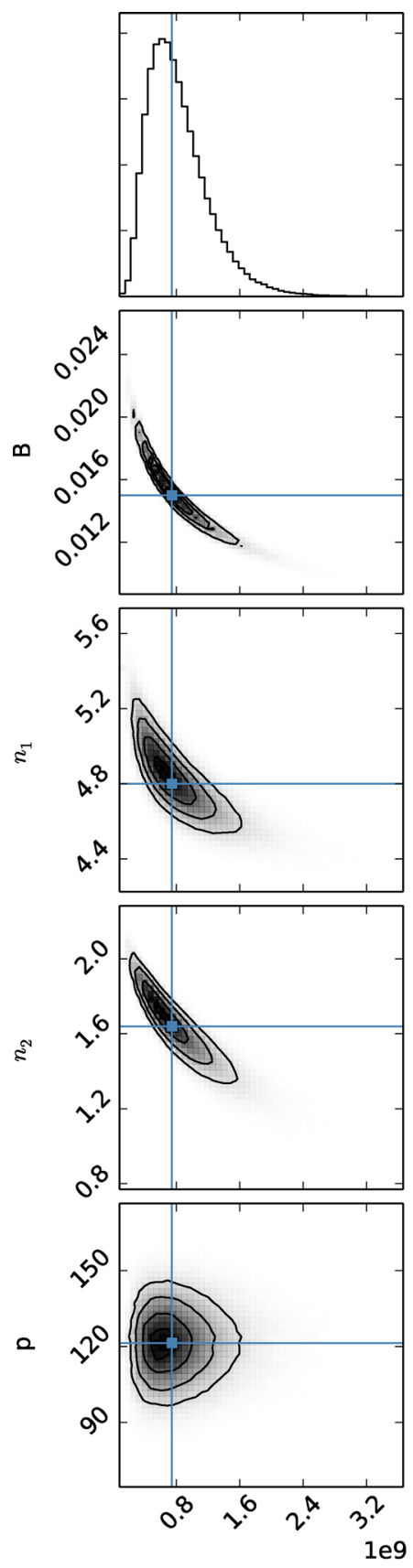

A
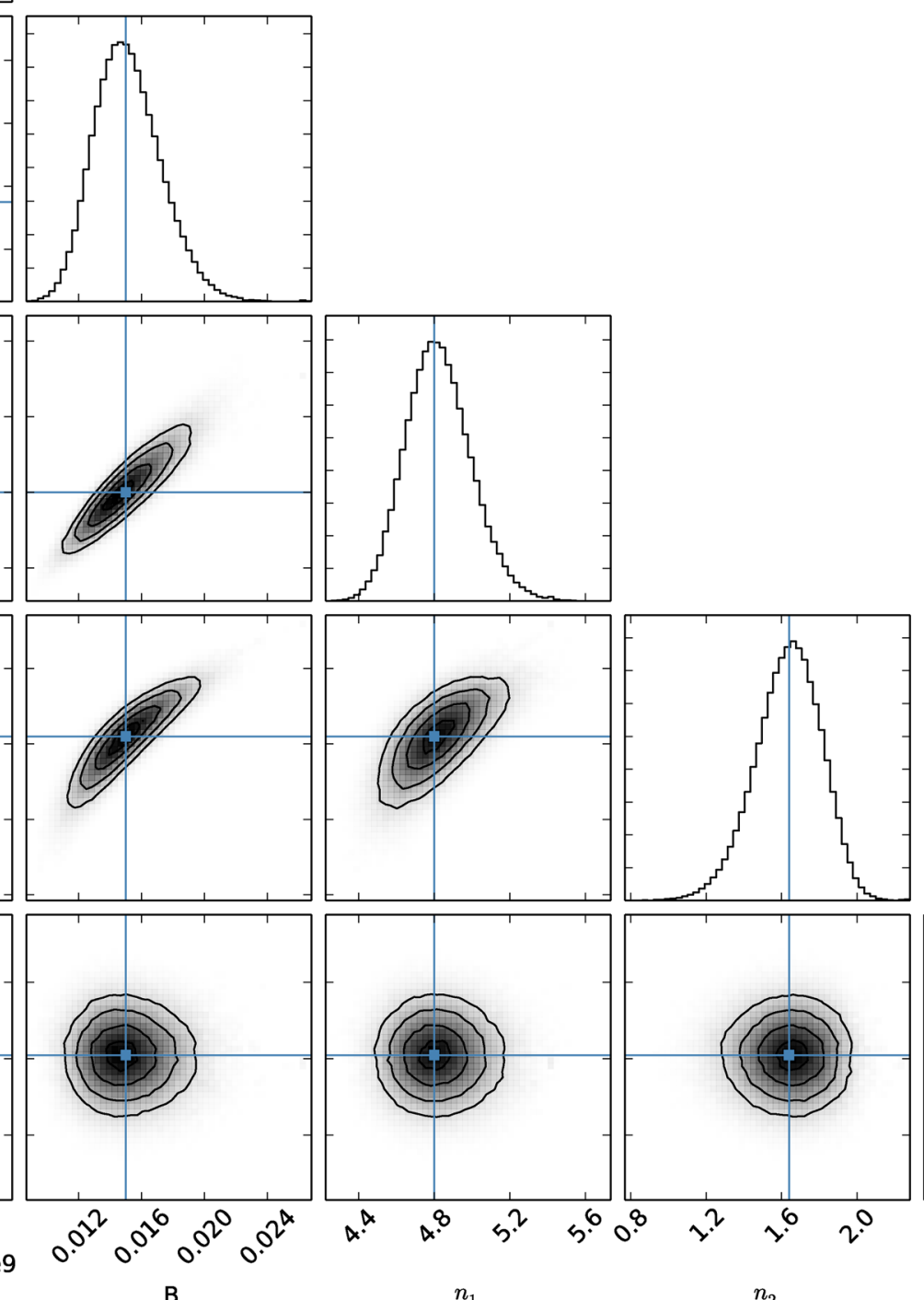

$n_{2}$

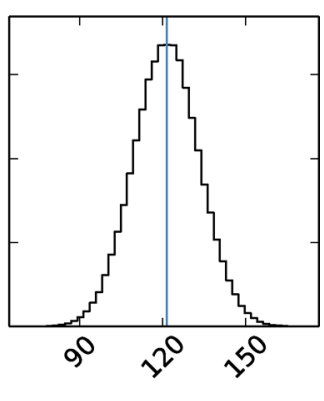

Figure B2. Same as Fig. B1, but for $545 \mathrm{GHz}$.

where

$$
\begin{aligned}
& W_{\text {mod., } \ell}^{(i p)(j q)}=\frac{1}{2 \ell+1} \sum_{m} w_{\ell m}^{(i p)}\left(w_{\text {mod. } \ell m}^{(j q)}\right)^{*}, \\
& W_{\text {mod. mod., } \ell}^{(i p)(j q)}=\frac{1}{2 \ell+1} \sum_{m} w_{\text {mod., } \ell m}^{(i p)}\left(w_{\text {mod., } \ell m}^{(j q)}\right)^{*},
\end{aligned}
$$

with

$w_{\text {mod., } \ell m}^{(i j)}=\int m^{2}(\hat{\boldsymbol{n}}) w^{i}(\hat{\boldsymbol{n}}) w^{j}(\hat{\boldsymbol{n}}) \mathrm{d} \hat{\boldsymbol{n}}$.

Here, we have assumed that the modulation is the same for all maps, so that $g^{i}(\hat{\boldsymbol{n}})$ carries the frequency dependence of the dust. We estimate the cross-power spectrum of $g^{i}$ and $g^{j}, C_{\ell}^{g, i j}$, from the best-fitting dust model in the likelihood, $\hat{C}_{\ell}^{\text {dust, } i j}$, as

$$
\sum_{\ell^{\prime}} M_{\ell \ell^{\prime}}\left[w^{i} \times w^{j}\right] \hat{C}_{\ell^{\prime}}^{\mathrm{dust}, i j}=\sum_{\ell^{\prime}} M_{\ell \ell^{\prime}}\left[m w^{i} \times m w^{j}\right] C_{\ell^{\prime}}^{g, i j},
$$

where the mask-coupling matrix $M_{\ell \ell^{\prime}}\left[m w^{i} \times m w^{j}\right]$ is calculated as in equation (A3) but with $W_{\ell}^{i j}$ replaced by the cross-spectrum of the modulated masks $m w^{i}$ and $m w^{j}$.

Fig. $\mathrm{C} 2$ shows the ratio of the square roots of the diagonal elements of the covariance matrices with and without the correction for dust modulation. We show results for both unbinned and binned spectra. The latter is sensitive to the enhanced mode-coupling across multipoles induced by the modulation. In this case, the diagonal errors are increased by about $20-40$ per cent, depending on mask and 
$\infty$
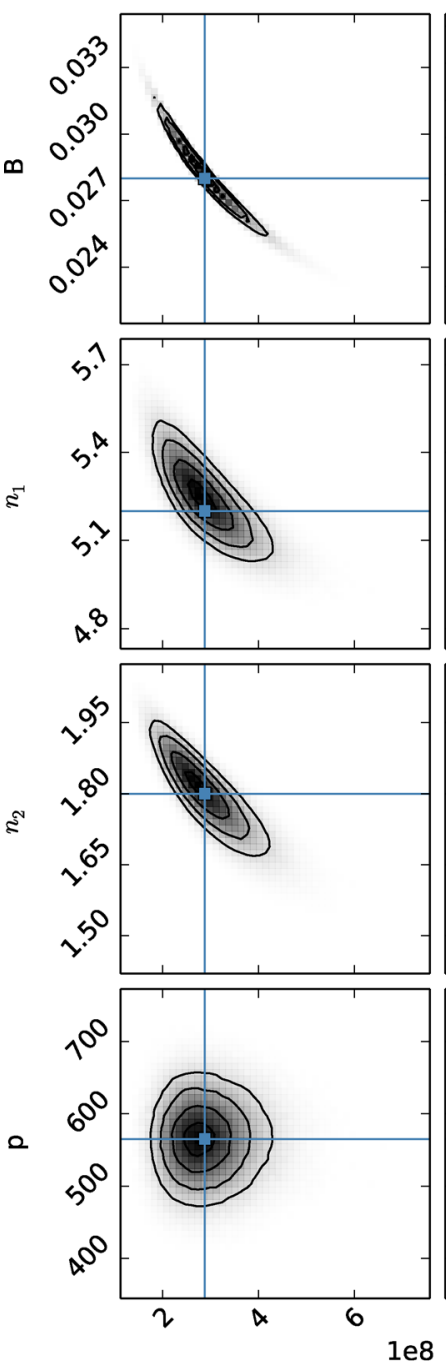

A
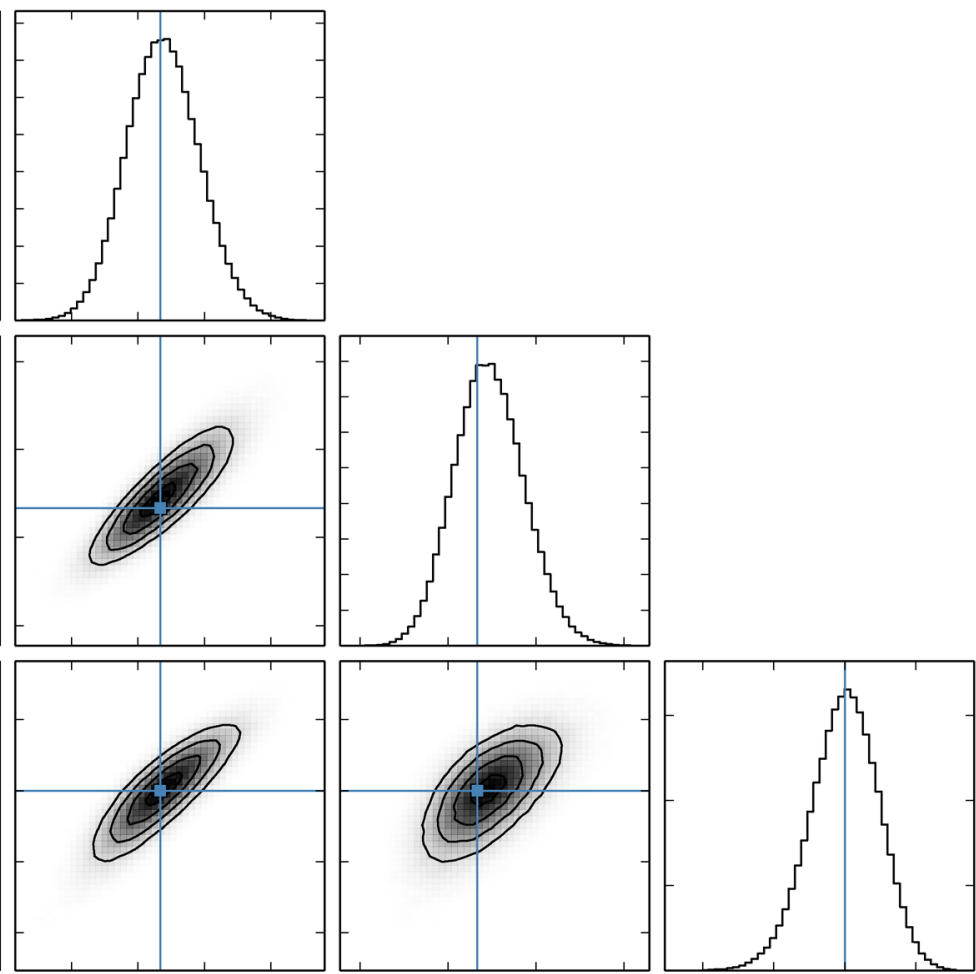

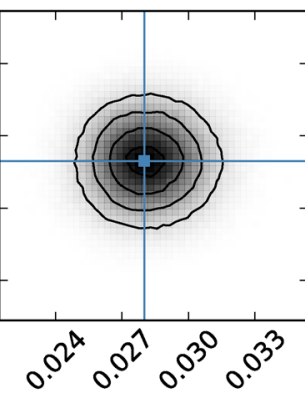

B

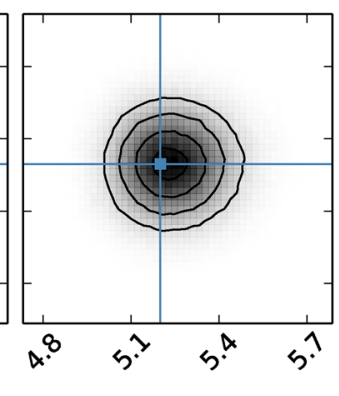

$n_{1}$

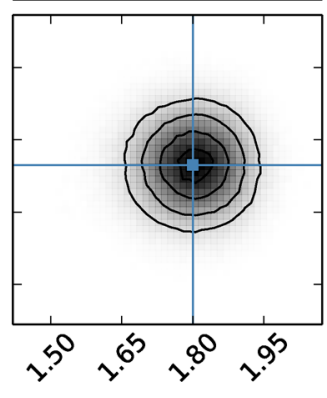

$n_{2}$

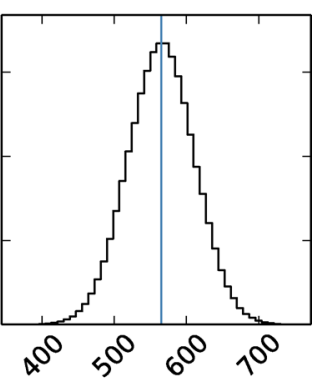

p

Figure B3. Same as Fig. B1, but for $857 \mathrm{GHz}$.

frequency, at $\ell<1000$ and drop to less than 5 per cent at higher multipoles. As expected, the effect of the modulation is greatest in cases where the dust is more dominant, i.e. large scales, high frequencies and masks that retain a larger fraction of the sky. The amplitude of the corrections roughly corresponds to our initial estimates of around 40 per cent.

\section{APPENDIX D: STABILITY AND ROBUSTNESS TESTS}

In this appendix, we investigate the stability of the distribution of the model parameters to technical choices that we make in the anal- ysis. These choices fall into two broad categories. The first involves the choices of fields, i.e. masks. The second involves the selection of data, such as number and type of spectra used. We performed a number of tests to investigate the impact of these choices on the parameters and to compare with the results of the baseline analysis used in most of this paper (six auto and cross-frequency spectra obtained by cross-correlating Year- 1 and Year- 2 maps outside mask 40 and with the union point source mask). The results are summarized in Fig. C3, which compares the CIB amplitudes $A^{\text {cib }}$ and CIB power-law index $\gamma^{\mathrm{cib}}$ for various analysis choices.

Increasing the sky fraction between 30 per cent and 50 per cent (i.e. using masks 30, 40, and 50) gives consistent results for the 

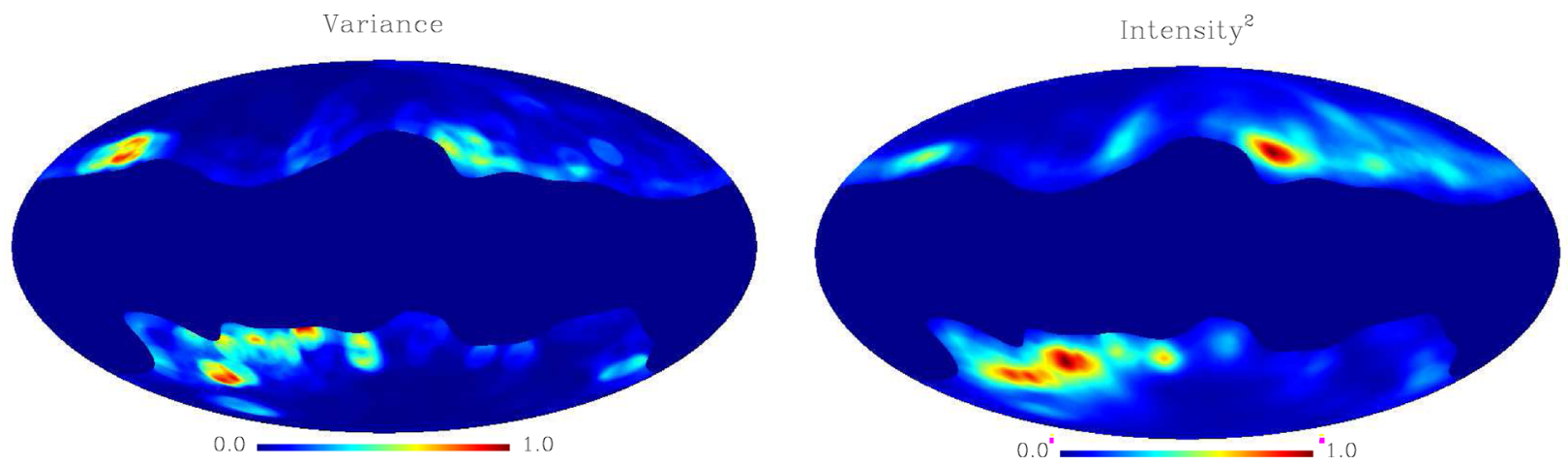

Figure C1. Left: normalized variance map of dust emission in circular apertures of $6^{\circ}$ radius constructed from smoothed $857 \mathrm{GHz}$ maps. This map traces the square of the modulation field. Right: square of the mean intensity constructed from the same procedure as for the variance map.
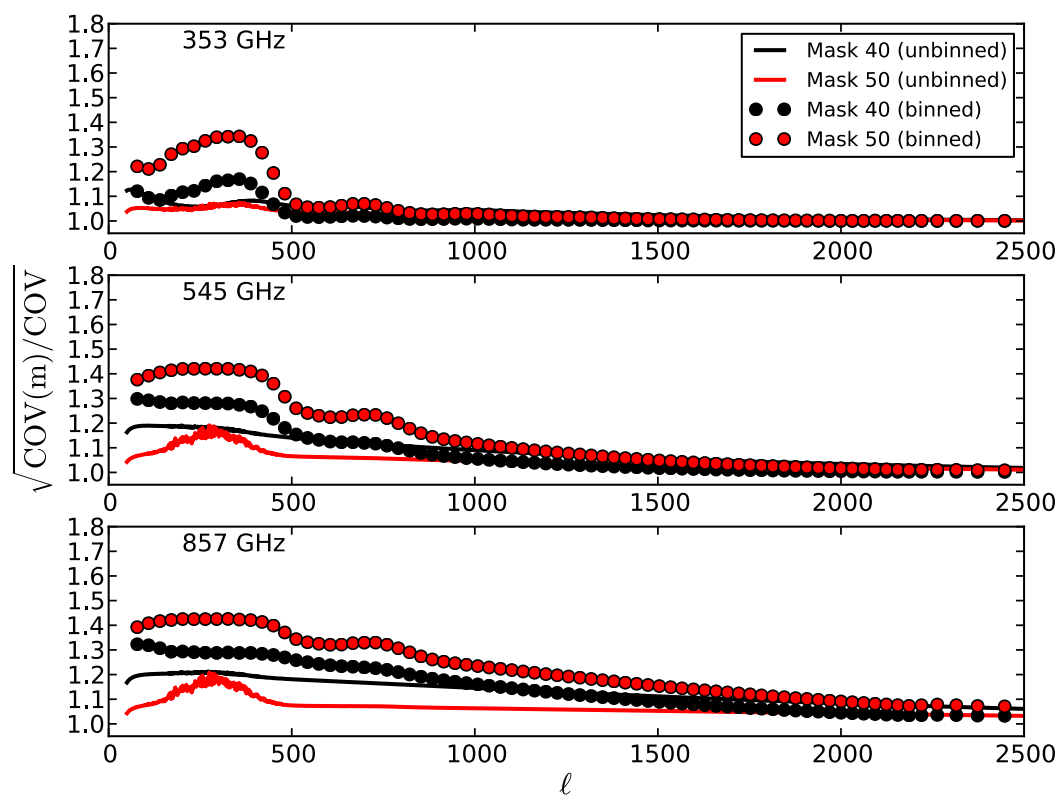

Figure C2. Ratio of the square roots of the diagonal elements of the covariance matrices with and without corrections for the statistical anisotropy of Galactic dust. The ratios are shown for the $353 \times 353$ (top), $545 \times 545$ (middle) and $857 \times 857$ (bottom) spectra for mask 40 (black) and mask 50 (red). In all cases, results for both unbinned (lines) and binned spectra (points) are shown.

CIB amplitudes, as already illustrated in Figs 11 and 12 and discussed in Section 5. In addition, switching to frequency-dependent point source masks, or changing to spectra constructed by crosscorrelating the first and second halves of the mission data, or detector-set maps, we see changes of less than $1 \sigma$ in the CIB parameters. Our best-fitting Poisson amplitudes are actually in very good agreement with the predictions of the B12 models, so imposing the B12 constraints on the point source amplitudes has very little effect (apart from shrinking the errors on the clustered CIB parameters). 

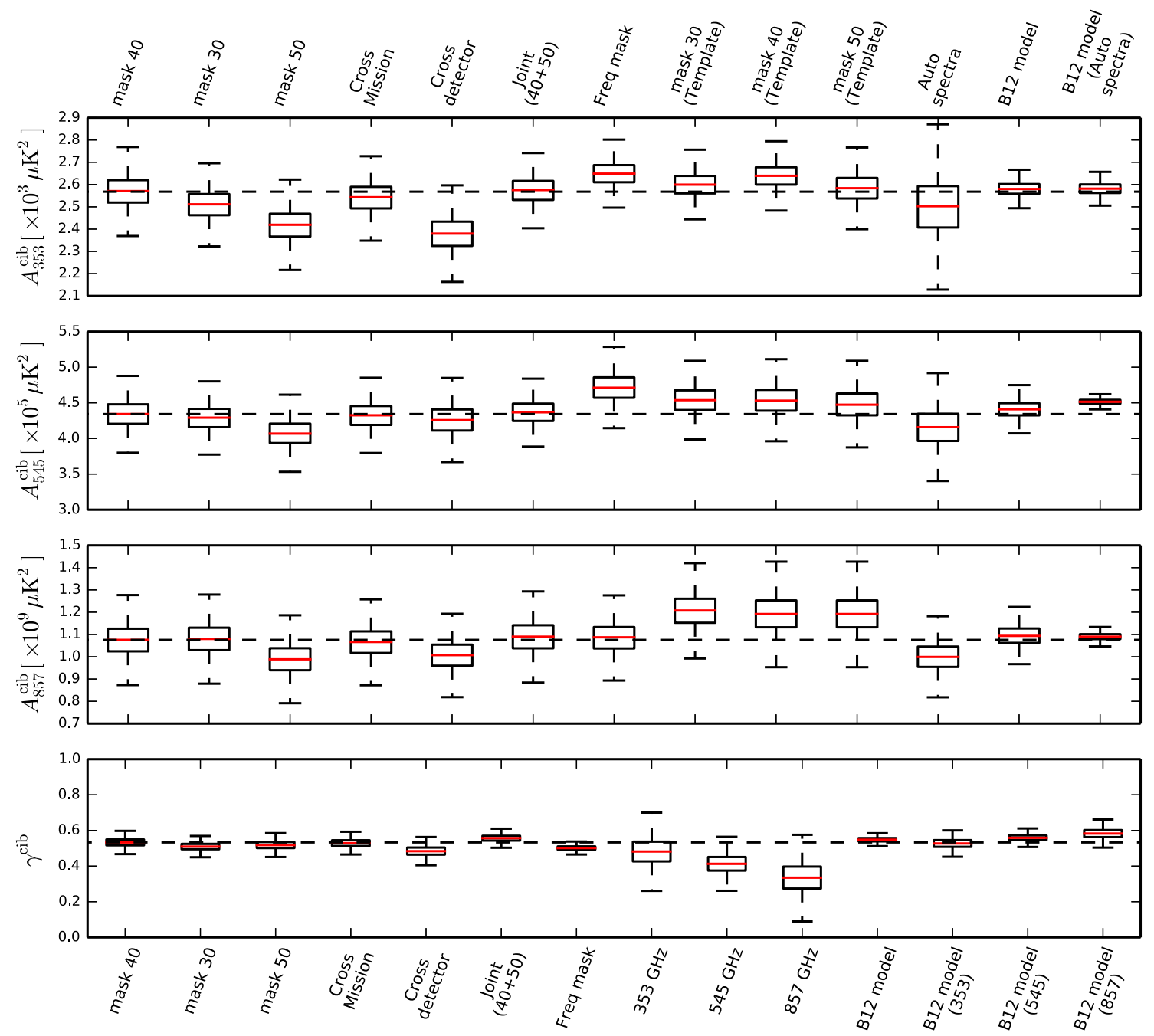

Figure C3. Comparison of the distributions of $A^{\text {cib }}$ and $\gamma^{\text {cib }}$ in the reference case (first data point in each panel) with a set of validation test cases. The red lines indicate the best-fitting values of the parameters. The boxes show the 68 per cent confidence interval and the outer lines show the 95 per cent interval. We use the results obtained with mask 40 (and the union point source mask), cross-correlating the Year-1 and Year-2 data, as our baseline (column 1). The other columns show variations with respect to this baseline. Common to all four panels: columns 2 and 3 shows the results using masks 30 and 50, respectively; column 4 shows results obtained by cross-correlating the first and second halves of the Planck mission data; column 5 shows results from cross-correlating the detector-set maps; column 6 shows the results of the joint-mask analysis (using masks 40 and 50); column 7 shows results using frequency-dependent point source masks. Common to top three panels $\left(A_{v}^{\text {cib }}\right)$ : columns $8-10$ show the results from replacing the power law-clustered CIB model with the halo model template for masks 30, 40 and 50, respectively; column 11 shows results when using auto-frequency spectra only; columns 12 and 13 show results with the Poisson amplitudes, $A^{\mathrm{ps}}$, kept fixed to B12 model predictions, using all six auto- and cross-frequency spectra (column 12) and only autofrequency spectra

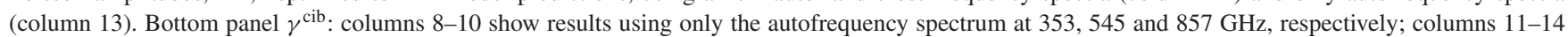
show results with the Poisson amplitudes, $A^{\mathrm{ps}}$, kept fixed to the B12 model predictions and using either all six auto and cross-frequency spectra (column 11) or only the autofrequency spectrum at each of 353,545 and $857 \mathrm{GHz}$ (columns 12-14, respectively).

This paper has been typeset from a $\mathrm{T}_{\mathrm{E}} \mathrm{X} / \mathrm{LAT} \mathrm{E}$ file prepared by the author. 